\title{
Stability threshold of two-dimensional Couette flow in Sobolev spaces
}

\author{
Nader Masmoudi and Weiren Zhao
}

\begin{abstract}
We study the stability threshold of the two-dimensional Couette flow in Sobolev spaces at high Reynolds number Re. We prove that if the initial vorticity $\Omega_{\text {in }}$ satisfies $\left\|\Omega_{\text {in }}-(-1)\right\|_{H^{\sigma}} \leq$ $\varepsilon \mathrm{Re}^{-1 / 3}$, then the solution of the two-dimensional Navier-Stokes equation approaches some shear flow which is also close to Couette flow for time $t \gg \mathrm{Re}^{1 / 3}$ by a mixing-enhanced dissipation effect, and then converges back to Couette flow when $t \rightarrow+\infty$.
\end{abstract}

\section{Introduction}

In this paper, we consider the two-dimensional incompressible Navier-Stokes equations in $\mathbb{T} \times \mathbf{R}$ :

$$
\left\{\begin{array}{l}
\partial_{t} V+V \cdot \nabla V+\nabla P-v \Delta V=0, \\
\nabla \cdot V=0, \\
\left.V\right|_{t=0}=V_{\text {in }}(x, y),
\end{array}\right.
$$

where $v$ denotes the viscosity, which is the multiplicative inverse of the Reynolds number Re. We denote by $V=\left(V^{1}, V^{2}\right)$ and $P$ the velocity and the pressure of the fluid respectively. Let $\Omega=\partial_{x} V^{2}-\partial_{y} V^{1}$ be the vorticity, which satisfies

$$
\Omega_{t}+V \cdot \nabla \Omega-v \Delta \Omega=0
$$

The Couette flow $(y, 0)$ is a steady solution of (1.1).

Now we introduce the perturbation: let $\Omega=\omega-1$ and $V=(y, 0)+\left(U^{x}, U^{y}\right)$; then $\omega=\partial_{x} U^{y}-\partial_{y} U^{x}$ satisfies

$$
\left\{\begin{array}{l}
\partial_{t} \omega+y \partial_{x} \omega-v \Delta \omega=-U \cdot \nabla \omega, \\
\left.\omega\right|_{t=0}=\omega_{\text {in }}(x, y),
\end{array}\right.
$$

and $U=\left(U^{x}, U^{y}\right)=\left(-\partial_{y} \psi, \partial_{x} \psi\right)$ with $\Delta \psi=\omega$.

The study of (1.3) for small perturbations is an old problem in hydrodynamic stability, considered by both Rayleigh ([37]) and Kelvin ([28]), as well as by many modern authors 
with new perspectives (see e.g. the classical texts $[19,45]$ and the references therein). Rayleigh and Kelvin both studied the linearization of (1.3), which is simply

$$
\left\{\begin{array}{l}
\partial_{t} \omega+y \partial_{x} \omega-v \Delta \omega=0 \\
\Delta \psi=\omega \\
\left.\omega\right|_{t=0}=\omega_{\text {in }}(x, y) .
\end{array}\right.
$$

Indeed, if we denote by $\widehat{\omega}(t, k, \eta)$ the Fourier transform of $\omega(t, x, y)$, then the solution of (1.4) can be written as

$$
\begin{aligned}
& \widehat{\omega}(t, k, \eta)=\widehat{\omega}_{\text {in }}(k, \eta+k t) \exp \left(-v \int_{0}^{t}|k|^{2}+|\eta-k s+k t|^{2} d s\right), \\
& \widehat{\psi}(t, k, \eta)=\frac{-\widehat{\omega}_{\text {in }}(k, \eta+k t)}{k^{2}+\eta^{2}} \exp \left(-v \int_{0}^{t}|k|^{2}+|\eta-k s+k t|^{2} d s\right),
\end{aligned}
$$

which gives

$$
\begin{gathered}
\left\|\partial_{y} P_{\neq} \psi\right\|_{L^{2}}+\langle t\rangle\left\|\partial_{x} P_{\neq} \psi\right\|_{L^{2}} \leq C\langle t\rangle^{-1} e^{-c v t^{3}}\left\|P_{\neq} \omega_{\text {in }}\right\|_{H^{2}} \\
\left\|P_{\neq} \omega\right\|_{L^{2}} \leq C\left\|P_{\neq} \omega_{\text {in }}\right\|_{L^{2}} e^{-c v t^{3}}
\end{gathered}
$$

where here we denote by $P_{\neq} f=f(x, y)-\frac{1}{2 \pi} \int_{\mathbb{T}} f(x, y) d x$ the projection to nonzero mode of $f$. The first inequality in (1.6) is the inviscid damping and the second one is the enhanced dissipation. These two results are both related to the vorticity mixing effect.

In [36], Orr observed the important phenomenon that the velocity will tend to 0 as $t \rightarrow \infty$, even for a time reversible system such as the Euler equations $(v=0)$. This phenomenon is so-called inviscid damping, which is the analogue in hydrodynamics of Landau damping found by Landau ([29]), which predicted the rapid decay of the electric field of the linearized Vlasov equation around homogeneous equilibrium. Mouhot and Villani ([35]) made a breakthrough and proved nonlinear Landau damping for perturbation in the Gevrey class (see also [8]). In this case, the mechanism leading to the damping is the vorticity mixing driven by shear flow or the Orr mechanism ([36]). See [2, 22, 38, 39, 44] for similar phenomena in various systems. We point out that inviscid damping for general shear flow is a challenge problem even at the linear level due to the presence of the nonlocal operator for general shear flow. For linear inviscid damping we refer to [20, 26, 41, 46] for the results of general monotone flows. For nonmonotone flows such as Poiseuille flow and Kolmogorov flow, another dynamic phenomenon should be taken into consideration, which is the so-called vorticity depletion phenomenon, predicted by Bouchet and Morita ([12]) and later proved by Wei, Zhang and Zhao ([42,43]). Due to possible nonlinear transient growth, it is a challenging task extending linear damping to nonlinear damping. Even for Couette flow there are only a few results. Moreover, nonlinear damping is sensitive to the topology of the perturbation. Indeed, Lin and Zeng ([32]) proved that nonlinear inviscid damping is not true for perturbation of Couette flow in $H^{s}$ for $s<\frac{3}{2}$. Bedrossian and Masmoudi ([7]) proved nonlinear inviscid damping around Couette flow 
when the perturbation is in Gevrey class 2_. Recently, Deng and Masmoudi ([17]) proved the instability for initial perturbations in Gevrey class $2_{+}$. We refer to [23, 25] and references therein for other related interesting results. We also refer to the very recent papers $[24,33]$, which show that nonlinear inviscid damping holds for general linear stable monotone shear flows. Moreover, it is also observed by Orr that, if we rewrite the linearized system using the change of coordinates $f(t, z, y)=\omega(t, z+t y, y)$, then the Fourier transform of the stream function $\phi(t, z, y)=\psi(t, z+y t, y)$ is

$$
\widehat{\phi}(t, k, \eta)=\frac{\hat{f}(t, k, \eta)}{(\eta-k t)^{2}+k^{2}} .
$$

The denominator of (1.7) is minimized at $t=\frac{\eta}{k}$ which is known as the Orr critical time.

The second phenomenon - enhanced dissipation - is sometimes referred to by modern authors as the "shear-diffusion mechanism". This decay rate is much faster than the diffusive decay of $e^{-v t}$. The mechanism leading to the enhanced dissipation is also due to vorticity mixing.

However, for the nonlinear system the Orr mechanism is known to interact poorly with the nonlinear term, creating a weakly nonlinear effect referred to as an echo. The basic mechanism is straightforward: a mode that is near its critical time is creating most of the velocity field and, at this point, it can interact with a part of enstrophy which is already mixed to transfer enstrophy to a mode which is un-mixing. When this third mode reaches its critical time, the result of the nonlinear interaction becomes very strong (the time delay explains the terminology "echo"). There are two necessary ways to control (compete against) the echo cascades. One is to assume enough smallness of the initial perturbations such that the rapid growth of the enstrophy may not happen before the enhanced dissipative time scale $v^{-\frac{1}{3}}$. The other is to assume enough regularity (Gevrey class) of the initial perturbations that one can pay enough regularity to control the growth caused by the echo cascade.

In this work, we are interested in the first method to stabilize the system and studying the long time behavior of (1.3) for small initial perturbations $\omega_{\text {in }}$. We aim to find the largest perturbation (threshold) in Sobolev spaces below which Couette flow is stable. More precisely, we are studying the following classical question:

Given a norm $\|\cdot\|_{X}$, find a $\mu=\mu(X)$ such that

$$
\begin{gathered}
\left\|\omega_{\mathrm{in}}\right\|_{X} \leq v^{\mu} \Rightarrow \text { stability, } \\
\left\|\omega_{\mathrm{in}}\right\|_{X} \gg v^{\mu} \Rightarrow \text { instability. }
\end{gathered}
$$

Another interesting question that is related to this problem is nonlinear enhanced dissipation and inviscid damping, which can be proposed in the following two ways:

1. Given a norm $\|\cdot\|_{X}\left(X \subset L^{2}\right)$, determine a $\mu=\mu(X)$ such that for $\left\|\omega_{\text {in }}\right\|_{X} \ll v^{\mu}$ and for $t>0$,

$$
\left\|\omega_{\neq}\right\|_{L_{x, y}^{2}} \leq C\left\|\omega_{\text {in }}\right\|_{X} e^{-c v^{\frac{1}{3}} t} \quad \text { and } \quad\left\|V_{\neq}\right\|_{L_{t, x, y}^{2}} \leq C\left\|\omega_{\text {in }}\right\|_{X},
$$


or the weak enhanced-dissipation-type estimate

$$
\left\|\omega_{\neq}\right\|_{L_{t}^{2} L_{x, y}^{2}} \leq C v^{-\frac{1}{6}}\left\|\omega_{\text {in }}\right\|_{X}
$$

holds for the Navier-Stokes equations (1.3).

2. Given $\mu$, is there an optimal function space $X \subset L^{2}$ so that if the initial vorticity satisfies $\left\|\omega_{\text {in }}\right\|_{X} \ll v^{\mu}$, then (1.8) or (1.9) holds for the Navier-Stokes equations (1.3)?

These two problems (find the smallest $\mu$ or find the largest function space $X$ ) are related to each other, since one can gain regularity in a short time by a standard time-weight argument if the initial perturbation is small enough.

We summarize the results as follows:

- For $\mu=0$, Bedrossian, Masmoudi and Vicol ([9]) showed that if $X$ is taken as Gevrey$m$ with $m<2$, then Couette flow is stable and (1.9) holds.

- For $\mu=\frac{1}{2}$, Bedrossian, Vicol and Wang ([10]) proved that Couette flow is stable, as well as nonlinear enhanced dissipation and inviscid damping for perturbation of initial vorticity in $H^{s}, s>1$.

- For $\mu=\frac{1}{2}$, recently in [34], we proved nonlinear enhanced dissipation and inviscid damping for perturbation of initial vorticity in the almost critical space $H_{x}^{\log } L_{y}^{2} \subset$ $L_{x, y}^{2}$.

Let us also mention some other recent progress on the stability problem of different types of shear flows in different domains:

- Three-dimensional Couette flow in $\mathbb{T} \times \mathbf{R} \times \mathbb{T}:$ If $X$ is taken as a Sobolev space, then $\mu=1$ gives stability $([3-5,40])$. Also see the very recent paper [15] for threedimensional Couette flow in a finite channel.

- Two-dimensional Couette flow in a finite channel: If $X$ is taken as a Sobolev space, then $\mu=\frac{1}{2}$ gives stability $([6,13,14,18])$.

- Other shear flows: See [16,20,21,30,31,43].

In this paper, we find a smaller $\mu\left(=\frac{1}{3}\right)$ such that Couette flow is stable, and nonlinear enhanced dissipation and inviscid damping hold, when $X$ is taken as a Sobolev space. Our main result is stated as follows:

Theorem 1.1. For $\sigma \geq 40$, $v>0$, there exist $0<\varepsilon_{0}, v_{0}<1$, such that for all $0<v \leq v_{0}$ and $0<\varepsilon \leq \varepsilon_{0}$, if $\omega_{\text {in }}$ satisfies $\left\|\omega_{\text {in }}\right\|_{H^{\sigma}} \leq \varepsilon \nu^{\frac{1}{3}}$, then the solution $\omega(t)$ of (1.3) with initial data $\omega_{\text {in }}$ satisfies the following properties:

1. Global stability in $H^{\sigma}$,

$$
\|\omega(t, x+t y+\Phi(t, y), y)\|_{H^{\sigma}} \leq C \varepsilon \mathcal{V}^{\frac{1}{3}},
$$

where $\Phi(t, y)$ is given explicitly by

$$
\Phi(t, y)=\int_{0}^{t} e^{\nu(t-\tau) \partial_{y}^{2}}\left(\frac{1}{2 \pi} \int_{\mathbb{T}} U^{x}(\tau, x, y) d x\right) d \tau .
$$


2. Inviscid damping,

$$
\left\|P_{\neq} U^{x}\right\|_{2}+\langle t\rangle\left\|U^{y} t\right\|_{2} \leq \frac{C \varepsilon v^{\frac{1}{3}}}{\left\langle v t^{3}\right\rangle}\langle t\rangle^{-1} .
$$

3. Weak enhanced dissipation,

$$
\left\|P_{\neq} \omega(t)\right\|_{2} \leq \frac{C \varepsilon v^{\frac{1}{3}}}{\left\langle v t^{3}\right\rangle} .
$$

The constant $C$ is independent of $v$ and $\varepsilon$.

Remark 1.2. By replacing $D(t, \eta)$ by $D(t, \eta)^{\alpha}$ with $\alpha \geq 1$ in the proof and assuming $\sigma$ large enough (depending on $\alpha$ ), one can obtain stronger enhanced dissipation of the following form:

$$
\left\|P_{\neq} \omega(t)\right\|_{2} \leq \frac{C \varepsilon v^{\frac{1}{3}}}{\left\langle v t^{3}\right\rangle^{\alpha}} .
$$

However, weak enhanced dissipation of the same decay rate as in Theorem 1.1 is enough for the proof of Sobolev stability. Both (1.12) and (1.13) are far from the exponential decay of the linear case.

It is natural to ask whether the exponent $\frac{1}{3}$ is sharp for stability with perturbations of finite regularity. In the proof, we show some evidence for the sharpness. There are two basic mechanisms leading to asymptotic stability: one is enhanced dissipation, the other is inviscid damping. Both will help us to estimate the accumulation of errors from the nonlinear interactions. We also face the following two facts: (1) Enhanced dissipation happens when $t \gg v^{-\frac{1}{3}}$. (2) We need to pay regularity to obtain inviscid damping. In order to prove stability with perturbation of finite regularity, we cannot use inviscid damping for free. So, roughly speaking, if we assume the perturbation is of size $\varepsilon v^{\mu}$, then the nonlinear term is of size $\varepsilon^{2} v^{2 \mu}$ and this error will accumulate in the time interval. This is the time interval before the enhanced dissipation starts. Thus, if we want the perturbation to stay of the same size as the initial data $\varepsilon v^{\mu}$, we need $\varepsilon^{2} v^{2 \mu-\frac{1}{3}} \leq \varepsilon v^{\mu}$, which gives us $\mu \geq \frac{1}{3}$. Of course, one can also allow the perturbation to grow to a different size. Indeed, from our proof (see (2.23)) one finds that the size of the coordinate system grows from $v^{\frac{1}{3}}$ to $v^{\frac{1}{6}}$.

For two-dimensional Couette flow in the finite channel $\mathbb{T} \times[-1,1]$, in [14] the authors proved that $\mu=\frac{1}{2}$ gives stability in Sobolev space, when the boundary effect is taken into consideration. It remains a very interesting problem whether the threshold can be improved to $\mu=\frac{1}{3}$ in the finite channel case.

Let us now outline the main ideas in the proof of Theorem 1.1. First, we provide a (well-chosen) change of variable that adapts to the solution as it evolves and yields a new "relative" velocity which is time integrable. Second, we will construct a new multiplier that can be regarded as a ghost weight in phase space and that will help us control the growth caused by echo cascades. 


\section{Proof of Theorem 1.1}

In this section we will present several key propositions and complete the proof of Theorem 1.1 by admitting those propositions.

\subsection{Notation and conventions}

See Section A.1 for the Fourier analysis conventions we are taking. A convention we generally use is to denote the discrete $x$ (or $z$ ) frequencies as subscripts. By convention we always use Greek letters such as $\eta$ and $\xi$ to denote frequencies in the $y$ or $v$ direction and lowercase Latin characters commonly used as indices such as $k$ and $l$ to denote frequencies in the $x$ or $z$ direction (which are discrete). Another convention we use is to denote $M, N, K$ as dyadic integers $M, N, K \in \mathbb{D}$, where

$$
\mathbb{D}=\left\{\frac{1}{2}, 1,2,4,8, \ldots, 2^{j}, \ldots\right\} .
$$

When a sum is written with indices $K, M, M^{\prime}, N$ or $N^{\prime}$ it will always be over a subset of $\mathbb{D}$. We will use the same $A$ for $A f=(A(\eta) \hat{f}(\eta))^{\vee}$ or $A \hat{f}=A(\eta) \hat{f}(\eta)$, where $A$ is a Fourier multiplier.

We use the notation $f \lesssim g$ when there exists a constant $C>0$ independent of the parameters of interest such that $f \leq C g$ (we define $g \gtrsim f$ analogously). Similarly, we use the notation $f \approx g$ when there exists $C>0$ such that $C^{-1} g \leq f \leq C g$.

We will denote the $l^{1}$ vector norm $|k, \eta|=|k|+|\eta|$, which by convention is the norm taken in our work. Similarly, given a scalar or vector in $\mathbf{R}^{n}$ we denote

$$
\langle v\rangle=\left(1+|v|^{2}\right)^{\frac{1}{2}}
$$

We use similar notation to denote the $x$ or $z$ average of a function:

$$
\langle f\rangle=\frac{1}{2 \pi} \int_{\mathbb{T}} f(x, y) d x=f_{0} .
$$

We also frequently use the notation $f_{\neq}=P_{\neq} f=f-f_{0}$. We denote the standard $L^{2}$ norms by $\|\cdot\|_{2}$. The norm of the Sobolev space $H^{\sigma}$ is given by

$$
\|f\|_{H^{\sigma}}=\left\|\left(\langle\eta\rangle^{\sigma} \hat{f}\right)^{\vee}\right\|_{2}
$$

The norm space-time Sobolev space $L_{T}^{p}\left(H^{\sigma}\right)$ is given by

$$
\|f\|_{L_{T}^{p}\left(H^{\sigma}\right)}= \begin{cases}\sup _{t^{\prime} \in[1, t]}\left\|f\left(t^{\prime}\right)\right\|_{H^{\sigma}}, & p=\infty, \\ \left(\int_{1}^{t}\left\|f\left(t^{\prime}\right)\right\|_{H^{\sigma}}^{p} d t^{\prime}\right)^{\frac{1}{p}}, & 1 \leq p<\infty .\end{cases}
$$

For $|m|=0,1,2, \ldots$, and $m \eta \geq 0$, let

$$
t_{m, \eta}=\frac{2 \eta}{2 m+1} \text {. }
$$


We then use

$$
I_{m, \eta} \stackrel{\text { def }}{=}\left[t_{m, \eta}, t_{m-1, \eta}\right]
$$

for $m=1,2, \ldots$, to denote any resonant interval and its left and right parts with $\eta \geq(2 m+1) m$. For $|\eta| \geq 3$, we denote by $E(\sqrt{|\eta|})$ the largest integer satisfying $(2 E(\sqrt{|\eta|})+1) E(\sqrt{|\eta|}) \leq|\eta|$ and then $E(\sqrt{|\eta|}) \approx \sqrt{|\eta|}$. Let $t(\eta)=\frac{2 \eta}{2 E(\sqrt{|\eta|})+1} \approx \sqrt{|\eta|}$ be the start of the resonant interval. Then we denote by

$$
I_{t}(\eta) \stackrel{\text { def }}{=}[t(\eta), 2|\eta|]=\bigcup_{m=1}^{E(\sqrt{\eta})} I_{m, \eta}
$$

the whole resonant interval.

For a statement $Q, 1_{Q}$ or $\chi^{Q}$ will denote the function that equals 1 if $Q$ is true and 0 otherwise.

\subsection{Coordinate transform}

We will use the same change of coordinates as in [9], which allows us to simultaneously "mod out" by the evolution of the time-dependent background shear flow and treat the mixing of this background shear as a perturbation of Couette flow (in particular, to understand the nonlinear effect of the Orr mechanism).

The change of coordinates used is $(t, x, y) \rightarrow(t, z, v)$, where $z(t, x, y)=x-t v(t, y)$ and $v(t, y)$ satisfies

$$
\left(\partial_{t}-v \partial_{y y}\right)(t(v(t, y)-y))=\left\langle U^{x}\right\rangle(t, y),
$$

with initial data $\lim _{t \rightarrow 0} t(v(t, y)-y)=0$, and where $\left\langle U^{x}\right\rangle(t, y)=\frac{1}{2 \pi} \int_{\mathbb{T}} U^{x}(t, x, y) d x$.

We define the following quantities:

$$
\begin{aligned}
C(t, v(t, y)) & =v(t, y)-y, \\
v^{\prime}(t, v(t, y)) & =\left(\partial_{y} v\right)(t, y), \\
v^{\prime \prime}(t, v(t, y)) & =\left(\partial_{y y} v\right)(t, y), \\
{\left[\partial_{t} v\right](t, v(t, y)) } & =\left(\partial_{t} v\right)(t, y), \\
f(t, z(t, x, y), v(t, y)) & =\omega(t, x, y), \\
\phi(t, z(t, x, y), v(t, y)) & =\psi(t, x, y), \\
\tilde{u}(t, z(t, x, y), v(t, y)) & =U^{x}(t, x, y) .
\end{aligned}
$$

Thus we get

$$
\Delta_{t} \phi \stackrel{\text { def }}{=} \partial_{z z} \phi+\left(v^{\prime}\right)^{2}\left(\partial_{v}-t \partial_{z}\right)^{2} \phi+v^{\prime \prime}\left(\partial_{v}-t \partial_{z}\right) \phi=f
$$

and

$$
\partial_{t} f+\left[\partial_{t} v\right] \partial_{v} f-v v^{\prime \prime} t \partial_{z} f+v^{\prime} \nabla_{z, v}^{\perp} P_{\neq} \phi \cdot \nabla_{z, v} f=v \Delta_{t} f
$$

where $\nabla_{z, v}^{\perp}=\left(-\partial_{v}, \partial_{z}\right), \nabla_{z, v}=\left(\partial_{z}, \partial_{v}\right), P_{\neq} \phi=\phi-\langle\phi\rangle, \tilde{u}_{0}(t, v)=\frac{1}{2 \pi} \int_{\mathbb{T}_{2 \pi}} \tilde{u}(t, z, v) d z$. 
We also obtain

$$
\partial_{t} \tilde{u}_{0}+\left[\partial_{t} v\right] \partial_{v} \tilde{u}_{0}+\left\langle v^{\prime} \nabla_{z, v}^{\perp} P_{\neq 0} \phi \cdot \nabla \tilde{u}\right\rangle=v \Delta_{t} \tilde{u}_{0} .
$$

Define the auxiliary function

$$
g(t, v)=\frac{1}{t}\left(\tilde{u}_{0}(t, v)-C(t, v)\right)
$$

which implies that

$$
\begin{gathered}
{\left[\partial_{t} v\right]=g+v v^{\prime \prime},} \\
v^{\prime} \partial_{v} C(t, v)=v^{\prime}(t, v)-1, \\
\partial_{t} C+\left[\partial_{t} v\right] \partial_{v} C=\left[\partial_{t} v\right], \\
v^{\prime} \partial_{v} v^{\prime}=v^{\prime \prime}=\Delta_{t} C,
\end{gathered}
$$

and that $g$ satisfies

$$
\partial_{t} g+\frac{2 g}{t}+g \partial_{v} g=-\frac{v^{\prime}}{t}\left\langle\nabla_{z, v}^{\perp} P_{\neq} \phi \cdot \nabla_{z, v} \tilde{u}\right\rangle+v\left(v^{\prime}\right)^{2} \partial_{v v} g .
$$

If we denote $h=v^{\prime}-1$, we get

$$
\partial_{t} h+g \partial_{v} h=\frac{-f_{0}-h}{t}+v \widetilde{\Delta}_{t} h .
$$

Let $\bar{h}=\frac{-f_{0}-h}{t} ;$ thus we obtain

$$
\partial_{t} \bar{h}+g \partial_{v} \bar{h}=-\frac{2}{t} \bar{h}+\frac{v^{\prime}}{t}\left\langle\nabla_{z, v}^{\perp} P_{\neq} \phi \cdot \nabla_{z, v} f\right\rangle+v \widetilde{\Delta}_{t} \bar{h} .
$$

It gives

$$
\partial_{t} f+u \cdot \nabla_{z, v} f=v \widetilde{\Delta}_{t} f
$$

where

$$
u(t, z, v)=\left(\begin{array}{l}
0 \\
g
\end{array}\right)+v^{\prime} \nabla_{z, v}^{\perp} P_{\neq} \phi
$$

and $\widetilde{\Delta}_{t} f=\partial_{z z} f+\left(v^{\prime}\right)^{2}\left(\partial_{v}-t \partial_{z}\right)^{2} f$.

By the change of the coordinates we reduce our problem to studying the following system:

$$
\left\{\begin{array}{l}
\partial_{t} f+u \cdot \nabla_{z, v} f=v \widetilde{\Delta}_{t} f, \\
u(t, z, v)=\left(\begin{array}{l}
0 \\
g
\end{array}\right)+v^{\prime} \nabla_{z, v}^{\perp} P_{\neq} \phi \\
\Delta_{t} \phi=f, \quad v^{\prime \prime}=v^{\prime} \partial_{v} v^{\prime}, \quad h=v^{\prime}-1,
\end{array}\right.
$$




$$
\left\{\begin{array}{l}
\partial_{t} g+\frac{2 g}{t}+g \partial_{v} g=-\frac{v^{\prime}}{t}\left\langle\nabla_{z, v}^{\perp} P_{\neq} \phi \cdot \nabla_{z, v} \tilde{u}\right\rangle+v\left(v^{\prime}\right)^{2} \partial_{v v} g \\
\partial_{t} \bar{h}+\frac{2}{t} \bar{h}+g \partial_{v} \bar{h}=\frac{v^{\prime}}{t}\left\langle\nabla_{z, v}^{\perp} P_{\neq} \phi \cdot \nabla_{z, v} f\right\rangle+v\left(v^{\prime}\right)^{2} \partial_{v v} \bar{h} \\
\partial_{t} h+g \partial_{v} h=\bar{h}+v\left(v^{\prime}\right)^{2} \partial_{v v} h \\
\tilde{u}=-v^{\prime}\left(\partial_{v}-t \partial_{z}\right) \phi .
\end{array}\right.
$$

\subsection{Main energy estimate}

In light of the previous section, our goal is to control the solution to (2.18) and (2.19) uniformly in a suitable norm as $t \rightarrow \infty$. The key idea we use for this is the carefully designed time-dependent norm written as

$$
\left\|A^{\sigma}(t, \nabla) f\right\|_{2}^{2}=\sum_{k} \int_{\eta}\left|A_{k}^{\sigma}(t, \eta) \hat{f}_{k}(t, \eta)\right|^{2} d \eta,
$$

where $A_{k}^{\sigma}(t, \eta)$ is defined in (3.9).

We also introduce another time-dependent norm for $8 \leq s \leq \sigma-10$ :

$$
\left\|A_{E}^{s}\left(t, \partial_{k}, \partial_{v}\right) f\right\|_{2}^{2}=\sum_{k \neq 0} \int_{\eta}\left|A_{E}^{s}(t, k, \eta) \hat{f}_{k}(t, \eta)\right|^{2} d \eta,
$$

which quantifies the enhanced dissipation effect with

$$
A_{E}^{s}(t, k, \eta)=\langle k, \eta\rangle^{s} D(t, \eta),
$$

with

$$
D(t, \eta)=\frac{1}{3} v|\eta|^{3}+\frac{1}{24} v\left(t^{3}-8|\eta|^{3}\right)_{+} .
$$

Here $E$ stands for enhanced dissipation.

We define our higher Sobolev energy:

$$
\mathcal{E}^{\sigma}(t)=\frac{1}{2}\left\|A^{\sigma}(t) f(t)\right\|_{2}^{2}+\varepsilon_{v}(t)
$$

where

$$
\varepsilon_{v}(t)=\|g\|_{H^{\sigma}}^{2}+v^{\frac{1}{3}}\|h\|_{H^{\sigma}}^{2}+v^{\frac{1}{3}}\|\bar{h}\|_{H^{\sigma}}^{2}+\|h\|_{H^{\sigma-1}}^{2}+\|\bar{h}\|_{H^{\sigma-1}}^{2} .
$$

By well-posedness theory for two-dimensional Navier-Stokes equations in Sobolev spaces we may safely ignore the time interval (say) $[0,1]$ by further restricting the size of the initial data. That is, we have the following lemma.

Lemma 2.1. For $\varepsilon>0, v>0$ and $\sigma \geq 40$, there exists $\varepsilon^{\prime}>0$ independent of $v$ such that if $\left\|\omega_{\text {in }}\right\|_{H^{\sigma}} \leq \varepsilon^{\prime} v^{\frac{1}{3}}$, then

$$
\sup _{t \in[0,1]} \mathcal{E}^{\sigma}(t) \leq\left(\varepsilon v^{\frac{1}{3}}\right)^{2}
$$


We define the following controls referred to in the sequel as the bootstrap hypotheses for $t \geq 1$.

\section{Higher regularity: main system.}

$$
\left\|A^{\sigma} f(t)\right\|_{2}^{2}+v \int_{1}^{t}\left\|\sqrt{-\Delta_{L}} A^{\sigma} f\left(t^{\prime}\right)\right\|_{2}^{2} d t^{\prime}+\int_{1}^{t} \mathrm{CK}_{w}\left(t^{\prime}\right) d t^{\prime} \leq\left(8 \varepsilon v^{\frac{1}{3}}\right)^{2},
$$

where the CK stands for "Cauchy-Kovalevskaya",

$$
\mathrm{CK}_{w}(t)=\sum_{k} \int \frac{\partial_{t} w_{k}(t, \eta)}{w_{k}(t, \eta)}\left|A_{k}^{\sigma}(t, \eta) \hat{f}_{k}(t, \eta)\right|^{2} d \eta
$$

\section{Higher regularity: coordinate system.}

$$
\begin{aligned}
& \langle t\rangle\|g\|_{H^{\sigma}}+\int_{1}^{t}\left\|g\left(t^{\prime}\right)\right\|_{H^{\sigma}} d t^{\prime} \leq 8 \varepsilon v^{\frac{1}{3}}, \\
& t^{3}\left\|A^{\sigma} \bar{h}(t)\right\|_{2}^{2}+\int_{1}^{t} t^{\prime 3}\left\|\sqrt{\frac{\partial_{t} w(\nabla)}{w(\nabla)}} A^{\sigma} \bar{h}\right\|_{2}^{2} d t^{\prime} \\
& \quad+\frac{1}{4} \int_{1}^{t} t^{\prime 2}\left\|A^{\sigma} \bar{h}\right\|_{2}^{2} d t^{\prime}+\frac{1}{4} v \int_{1}^{t} t^{\prime 3}\left\|\partial_{v} A^{\sigma} \bar{h}\right\|_{2}^{2} d t^{\prime} \leq 8 \varepsilon\left(\varepsilon v^{\frac{1}{6}}\right)^{2}, \\
& \|h(t)\|_{H^{\sigma}}^{2}+v \int_{1}^{t}\left\|\partial_{v} h\left(t^{\prime}\right)\right\|_{H^{\sigma}}^{2} d t^{\prime} \leq 8\left(10 \varepsilon v^{\frac{1}{6}}\right)^{2} .
\end{aligned}
$$

\section{Lower regularity: enhanced dissipation.}

$$
\left\|A_{E}^{S} f(t)\right\|_{2}^{2}+\frac{2}{5} v \int_{1}^{t}\left\|\sqrt{-\Delta_{L}} A_{E}^{s} f\left(t^{\prime}\right)\right\|_{2}^{2} d t^{\prime} \leq\left(8 \varepsilon v^{\frac{1}{3}}\right)^{2} .
$$

\section{Lower regularity: decay of the zero mode.}

$$
\begin{aligned}
& \langle t\rangle^{4}\|g(t)\|_{H^{\sigma-6}}^{2}+v \int_{1}^{t} t^{\prime 4}\left\|\partial_{v} g\left(t^{\prime}\right)\right\|_{H^{\sigma-6}}^{2} d t^{\prime} \leq\left(8 \varepsilon v^{\frac{1}{3}}\right)^{2}, \\
& \langle t\rangle^{4}\|\bar{h}(t)\|_{H^{\sigma-6}}^{2}+v \int_{1}^{t} t^{\prime 4}\left\|\partial_{v} \bar{h}\left(t^{\prime}\right)\right\|_{H^{\sigma-6}}^{2} d t^{\prime} \leq\left(8 \varepsilon v^{\frac{1}{3}}\right)^{2}, \\
& \left\|f_{0}\right\|_{H^{s}}^{2}+\frac{t v}{2}\left\|\partial_{v} f_{0}\right\|_{H^{s}}^{2}+v \int_{1}^{t}\left(\left\|\partial_{v} f_{0}\left(t^{\prime}\right)\right\|_{H^{s}}^{2}+\frac{t^{\prime} v}{2}\left\|\partial_{v} f_{0}\left(t^{\prime}\right)\right\|_{H^{s}}^{2}\right) d t^{\prime} \leq\left(8 \varepsilon v^{\frac{1}{3}}\right)^{2} .
\end{aligned}
$$

\section{Assistant estimates.}

$$
\begin{aligned}
& \langle t\rangle\|\bar{h}\|_{H^{\sigma-1}}+\int_{1}^{t}\left\|\bar{h}\left(t^{\prime}\right)\right\|_{H^{\sigma-1}} d t^{\prime} \leq 8 \varepsilon v^{\frac{1}{3}}, \\
& \|h(t)\|_{H^{\sigma-1}}^{2}+v \int_{1}^{t}\left\|\partial_{v} h\left(t^{\prime}\right)\right\|_{H^{\sigma-1}}^{2} d t^{\prime} \leq 8\left(10 \varepsilon v^{\frac{1}{3}}\right)^{2} .
\end{aligned}
$$


The next proposition follows from the bootstrap hypotheses, elliptic estimates and the properties of the multipliers $A^{\sigma}$ and $A_{E}^{s}$.

Proposition 2.2. Under the bootstrap hypotheses, the following inequalities hold:

$$
\begin{aligned}
& \|f\|_{H^{\sigma}}+v^{\frac{1}{2}}\left\|\sqrt{-\Delta_{L}} f\right\|_{L_{T}^{2}\left(H^{\sigma}\right)}+\left\|\sqrt{\frac{\partial_{t} w(\nabla)}{w(\nabla)} f}\right\|_{L_{T}^{2}\left(H^{\sigma}\right)} \lesssim \varepsilon v^{\frac{1}{3}}, \\
& \left\|f_{\neq}\right\|_{H^{s}}+v^{\frac{1}{2}}\left\|\sqrt{-\Delta_{L}} f_{\neq}\right\|_{L_{T}^{2}\left(H^{s}\right)} \lesssim \frac{\varepsilon v^{\frac{1}{3}}}{\left\langle v t^{3}\right\rangle},
\end{aligned}
$$

and the inviscid damping results

$$
\left\|P_{\neq} \phi\right\|_{H^{\sigma-4}} \lesssim \frac{\varepsilon \nu^{\frac{1}{3}}}{\left\langle t^{2}\right\rangle}, \quad\left\|\tilde{u}_{\neq}\right\|_{H^{\sigma-3}} \lesssim \frac{\varepsilon \nu^{\frac{1}{3}}}{\langle t\rangle} .
$$

This proposition together with Lemma A.2 implies Theorem 1.1.

Proof. By Lemma 3.3, we get $A_{k}^{\sigma}(t, \eta) \approx\langle k, \eta\rangle^{\sigma}$. Thus we have $\left\|A^{\sigma} f\right\|_{2} \approx\|f\|_{H^{\sigma}}$ which implies (2.27).

By Lemma 3.4, we get $D(t, \eta) \gtrsim v t^{3}$, thus $\left\|A_{E}^{s} f\right\|_{2} \gtrsim v t^{3}\|f\|_{H^{s}}$ which gives (2.28).

The inviscid damping result (2.29) follows from Lemmas 4.1 and 4.2.

For enhanced dissipation and inviscid damping in the Sobolev norm, we also have the following remark.

Remark 2.3. Under the bootstrap hypotheses, it holds that

$$
\left\|\omega_{\neq}(t, x+t y+\Phi(t, y), y)\right\|_{H^{s}} \lesssim \frac{\varepsilon v^{\frac{1}{3}}}{\left\langle v t^{3}\right\rangle}
$$

and

$$
\begin{aligned}
\left\|U^{y}(t, x+t y+\Phi(t, y), y)\right\|_{H^{\sigma-4}} \lesssim \frac{\varepsilon v^{\frac{1}{3}}}{\left\langle t^{2}\right\rangle}, \\
\left\|U_{\neq}^{x}(t, x+t y+\Phi(t, y), y)\right\|_{H^{\sigma-4}} \lesssim \frac{\varepsilon v^{\frac{1}{3}}}{\langle t\rangle} .
\end{aligned}
$$

Recall that

$$
\begin{gathered}
f(t, z(t, x, y), v(t, y))=\omega(t, x, y) \\
\quad \Rightarrow \omega(t, x+t y+\Phi(t, y), y)=f(t, x, v(t, y)), \\
\tilde{u}(t, z(t, x, y), v(t, y))=U^{x}(t, x, y) \\
\quad \Rightarrow U^{x}(t, x+t y+\Phi(t, y), y)=\tilde{u}(t, x, v(t, y)), \\
\partial_{z} \phi(t, z(t, x, y), v(t, y))=U^{y}(t, x, y) \\
\quad \Rightarrow U^{y}(t, x+t y+\Phi(t, y), y)=\left(\partial_{z} \phi\right)(t, x, v(t, y)) .
\end{gathered}
$$

The remark follows directly from (2.28), (2.29), the composition Lemma A.2 and the bootstrap hypotheses for the regularity of the coordinate system. 
By Lemma 2.1, for the rest of the proof we may focus on times $t \geq 1$. Let $I^{*}$ be the connected set of times $t \geq 1$ such that the bootstrap hypotheses (2.22)-(2.26) are all satisfied. We will work on regularized solutions for which we know $\mathcal{E}^{\sigma}(t)$ takes values continuously in time, and hence $I^{*}$ is a closed interval $\left[1, T^{*}\right]$ with $T^{*} \geq 1$. The bootstrap is complete if we show that $I^{*}$ is also open, which is the purpose of the following proposition, the proof of which constitutes the majority of this work.

Proposition 2.4. For $\sigma \geq 40, v>0$ and $8 \leq s \leq \sigma-10$, there exist $0<\varepsilon_{0}$, $v_{0}<1$, such that for all $0<v \leq v_{0}$ and $0<\varepsilon \leq \varepsilon_{0}$, such that if on $\left[1, T^{*}\right]$ the bootstrap hypotheses (2.22)-(2.26) hold, then for any $t \in\left[1, T^{*}\right]$, we have the following properties:

1. Vorticity boundedness:

$$
\left\|A^{\sigma} f(t)\right\|_{2}^{2}+v \int_{1}^{t}\left\|\sqrt{-\Delta_{L}} A^{\sigma} f\left(t^{\prime}\right)\right\|_{2}^{2} d t^{\prime}+\int_{1}^{t} \mathrm{CK}_{w}\left(t^{\prime}\right) d t^{\prime} \leq\left(6 \varepsilon v^{\frac{1}{3}}\right)^{2} .
$$

2. Control of coordinates system:

$$
\begin{aligned}
& \langle t\rangle\|g\|_{H^{\sigma}}+\int_{1}^{t}\left\|g\left(t^{\prime}\right)\right\|_{H^{\sigma}} d t^{\prime} \leq 6 \varepsilon v^{\frac{1}{3}}, \\
& t^{3}\left\|A^{\sigma} \bar{h}(t)\right\|_{2}^{2}+\int_{1}^{t} t^{\prime 3}\left\|\sqrt{\frac{\partial_{t} w}{w}} \bar{h}\right\|_{H^{\sigma}}^{2} d t^{\prime} \\
& \quad+\frac{1}{4} \int_{1}^{t} t^{\prime 2}\left\|A^{\sigma} \bar{h}\right\|_{2}^{2} d t^{\prime}+\frac{1}{4} v \int_{1}^{t} t^{\prime 3}\left\|\partial_{v} A^{\sigma} \bar{h}\right\|_{2}^{2} d t^{\prime} \leq 6 \varepsilon\left(\varepsilon v^{\frac{1}{6}}\right)^{2}, \\
& \|h(t)\|_{H^{\sigma}}^{2}+v \int_{1}^{t}\left\|\partial_{v} h\left(t^{\prime}\right)\right\|_{H^{\sigma}}^{2} d t^{\prime} \leq 6\left(10 \varepsilon v^{\frac{1}{6}}\right)^{2} .
\end{aligned}
$$

3. Enhanced dissipation:

$$
\left\|A_{E}^{s} f(t)\right\|_{2}^{2}+\frac{2}{5} v \int_{1}^{t}\left\|\sqrt{-\Delta_{L}} A_{E}^{s} f\left(t^{\prime}\right)\right\|_{2}^{2} d t^{\prime} \leq\left(6 \varepsilon v^{\frac{1}{3}}\right)^{2},
$$

4. Decay of the zero mode:

$$
\begin{aligned}
& \langle t\rangle^{4}\|g(t)\|_{H^{\sigma-6}}^{2}+v \int_{1}^{t} t^{\prime 4}\left\|\partial_{v} g\left(t^{\prime}\right)\right\|_{H^{\sigma-6}}^{2} d t \leq\left(6 \varepsilon v^{\frac{1}{3}}\right)^{2}, \\
& \langle t\rangle^{4}\|\bar{h}(t)\|_{H^{\sigma-6}}^{2}+v \int_{1}^{t} t^{\prime 4}\left\|\partial_{v} \bar{h}\left(t^{\prime}\right)\right\|_{H^{\sigma-6}}^{2} d t \leq\left(6 \varepsilon v^{\frac{1}{3}}\right)^{2}, \\
& \left\|f_{0}(t)\right\|_{H^{s}}^{2}+\frac{t v}{2}\left\|\partial_{v} f_{0}\right\|_{H^{s}}^{2}+v \int_{1}^{t}\left(\left\|\partial_{v} f_{0}(t)\right\|_{H^{s}}^{2}+\frac{t^{\prime} v}{2}\left\|\partial_{v} f_{0}\left(t^{\prime}\right)\right\|_{H^{s}}^{2}\right) d t^{\prime} \leq\left(6 \varepsilon v^{\frac{1}{3}}\right)^{2} .
\end{aligned}
$$

5. Assistant estimate:

$$
\begin{aligned}
& \langle t\rangle\|\bar{h}\|_{H^{\sigma-1}}+\int_{1}^{t}\left\|\bar{h}\left(t^{\prime}\right)\right\|_{H^{\sigma-1}} d t^{\prime} \leq 6 \varepsilon v^{\frac{1}{3}}, \\
& \|h(t)\|_{H^{\sigma-1}}^{2}+v \int_{1}^{t}\left\|\partial_{v} h\left(t^{\prime}\right)\right\|_{H^{\sigma-1}}^{2} d t^{\prime} \leq 6\left(10 \varepsilon v^{\frac{1}{3}}\right)^{2},
\end{aligned}
$$

from which it follows that $T^{*}=+\infty$. 
The remainder of the paper is devoted to the proof of Proposition 2.4, the primary step being to show that on $\left[1, T^{*}\right]$, we have the following estimates:

$$
\begin{aligned}
& \left\|A^{\sigma} f(t)\right\|_{2}^{2}+v \int_{1}^{t}\left\|\sqrt{-\Delta_{L}} A^{\sigma} f\left(t^{\prime}\right)\right\|_{2}^{2} d t^{\prime}+\int_{1}^{t} \mathrm{CK}_{w}\left(t^{\prime}\right) d t^{\prime} \\
& \leq 2\left\|A^{\sigma} f(1)\right\|_{2}^{2}+C \varepsilon^{3} v^{\frac{2}{3}} \\
& \langle t\rangle\|g\|_{H^{\sigma}}+\int_{1}^{t}\left\|g\left(t^{\prime}\right)\right\|_{H^{\sigma}} d t^{\prime} \leq 2\|g(1)\|_{H^{\sigma}}+C \varepsilon^{2} v^{\frac{1}{3}}, \\
& t^{3}\left\|A^{\sigma} \bar{h}(t)\right\|_{2}^{2}+\int_{1}^{t} t^{\prime 3}\left\|\sqrt{\frac{\partial_{t} w}{w}} \bar{h}\right\|_{H^{\sigma}}^{2} d t^{\prime}+\frac{1}{4} \int_{1}^{t} t^{\prime 2}\left\|A^{\sigma} \bar{h}\right\|_{2}^{2} d t^{\prime} \\
& +\frac{1}{4} v \int_{1}^{t} t^{\prime 3}\left\|\partial_{v} A^{\sigma} \bar{h}\right\|_{2}^{2} d t^{\prime} \leq 2\|\bar{h}(1)\|_{H^{\sigma}}^{2}+C \varepsilon^{4} v^{\frac{1}{3}}, \\
& \|h(t)\|_{H^{\sigma}}^{2}+v \int_{1}^{t}\left\|\partial_{v} h\left(t^{\prime}\right)\right\|_{H^{\sigma}}^{2} d t^{\prime} \leq 2\|h(1)\|_{H^{\sigma}}^{2}+C \varepsilon^{3} v^{\frac{1}{3}}, \\
& \left\|A_{E}^{s} f(t)\right\|_{2}^{2}+\frac{2}{5} v \int_{1}^{t}\left\|\sqrt{-\Delta_{L}} A_{E}^{s} f\left(t^{\prime}\right)\right\|_{2}^{2} d t^{\prime} \leq 2\left\|A_{E}^{s} f(1)\right\|_{2}^{2}+C \varepsilon^{3} v^{\frac{2}{3}}, \\
& \langle t\rangle^{4}\|g(t)\|_{H^{\sigma-6}}^{2}+v \int_{1}^{t} t^{\prime 4}\left\|\partial_{v} g\left(t^{\prime}\right)\right\|_{H^{\sigma-6}}^{2} d t^{\prime} \leq 2\|g(1)\|_{H^{\sigma-6}}^{2}+C \varepsilon\left(\varepsilon v^{\frac{1}{3}}\right)^{2}, \\
& \langle t\rangle^{4}\|\bar{h}(t)\|_{H^{\sigma-6}}^{2}+v \int_{1}^{t} t^{\prime 4}\left\|\partial_{v} \bar{h}\left(t^{\prime}\right)\right\|_{H^{\sigma-6}}^{2} d t \leq 2\|\bar{h}(1)\|_{H^{\sigma-6}}^{2}+C \varepsilon\left(\varepsilon v^{\frac{1}{3}}\right)^{2}, \\
& \left\|f_{0}(t)\right\|_{H^{s}}^{2}+\frac{t v}{2}\left\|\partial_{v} f_{0}\right\|_{H^{s}}^{2}+v \int_{1}^{t}\left(\left\|\partial_{v} f_{0}(t)\right\|_{H^{s}}^{2}+\frac{t^{\prime} v}{2}\left\|\partial_{v} f_{0}\left(t^{\prime}\right)\right\|_{H^{s}}^{2}\right) d t^{\prime} \\
& \leq 2\left\|f_{0}(1)\right\|_{H^{s}}^{2}+v\left\|\partial_{v} f_{0}(1)\right\|_{H^{s}}^{2}+C \varepsilon^{3} v^{\frac{2}{3}}, \\
& \langle t\rangle\|\bar{h}\|_{H^{\sigma-1}}+\int_{1}^{t}\left\|\bar{h}\left(t^{\prime}\right)\right\|_{H^{\sigma-1}} d t^{\prime} \leq 2\|\bar{h}(1)\|_{H^{\sigma-1}}+C \varepsilon^{2} v^{\frac{1}{3}}, \\
& \|h(t)\|_{H^{\sigma-1}}^{2}+v \int_{1}^{t}\left\|\partial_{v} h\left(t^{\prime}\right)\right\|_{H^{\sigma-1}}^{2} d t^{\prime} \leq 2\|h(1)\|_{H^{\sigma-1}}^{2}+8\|\bar{h}\|_{L_{T}^{1}\left(H^{\sigma-1}\right)}^{2}+C \varepsilon^{3} v^{\frac{2}{3}},
\end{aligned}
$$

for some constant $C$ independent of $\varepsilon, v$ and $T^{*}$. If $\varepsilon$ is sufficiently small then (2.30)(2.39) implies Proposition 2.4.

It is natural to compute the time evolution of the following quantities:

$$
\varepsilon_{H, f}=\left\|A^{\sigma} f(t)\right\|_{2}^{2}, \quad \varepsilon_{H, g}=t\|g\|_{H^{\sigma}}, \quad \varepsilon_{H, \bar{h}}=t^{3}\left\|A^{\sigma} \bar{h}\right\|_{H^{\sigma}}^{2}, \quad \varepsilon_{H, h}=\|h(t)\|_{H^{\sigma}}^{2}
$$
and

$$
\begin{gathered}
\mathcal{E}_{L, \neq}=\left\|A_{E}^{s} f(t)\right\|_{2}^{2}, \quad \mathcal{E}_{L, g}=t^{4}\|g(t)\|_{H^{\sigma-6}}^{2}, \quad \mathcal{E}_{L, \bar{h}}=t^{4}\|\bar{h}\|_{H^{\sigma-6}}^{2}, \\
\mathcal{E}_{L, 0}=\left\|f_{0}(t)\right\|_{H^{s}}^{2}+\frac{t v}{2}\left\|\partial_{v} f_{0}\right\|_{H^{s}}^{2}
\end{gathered}
$$

and

$$
\varepsilon_{\mathrm{as}, \bar{h}}=t^{2}\|\bar{h}\|_{H^{\sigma-1}}^{2}, \quad \varepsilon_{\mathrm{as}, h}=\|h(t)\|_{H^{\sigma-1}}^{2},
$$


where $H$ stands for the highest regularity, $L$ stands for the lower regularity and "as" stands for assistant.

The most difficult part in the proof is to control the energy $\varepsilon_{H, f}$. Here we present the calculations of the time evolution of $\varepsilon_{H, f}$. The calculations of the time evolution of $\varepsilon_{H, g}$, $\mathcal{E}_{\mathrm{as}, \bar{h}}, \mathcal{E}_{\mathrm{as}, h}, \mathcal{E}_{H, \bar{h}}$ and $\mathcal{E}_{H, h}$ are in Section 9.1. The calculations of the time evolution of $\mathcal{E}_{L, g}$ and $\mathcal{E}_{L, \bar{h}}$ are in Section 9.3. The calculations of the time evolution of $\mathcal{E}_{L, \neq}$ and $\mathcal{E}_{L, 0}$ are in Section 10.

The rest of this section will give an outline of the proof of (2.30).

The proof of (2.31) can be found in Section 9.1.1.

The proofs of (2.32) and (2.33) can be found in Section 9.2.

The proof of (2.34) can be found in Section 10.1.

The proof of (2.35) can be found in Section 9.3.1.

The proof of (2.36) can be found in Section 9.3.2.

The proof of (2.37) can be found in Section 10.2.

The proofs of (2.38) and (2.39) can be found in Section 9.1.2.

From the time evolution of $\varepsilon_{H, f}$ we get

$$
\begin{aligned}
& \frac{1}{2} \frac{d}{d t} \int_{\mathbb{T} \times \mathbf{R}} \mid A^{\sigma} f(t) \|^{2} d v d z \\
& \quad=-\mathrm{CK}_{w}-\int A^{\sigma} f A^{\sigma}(u \nabla f) d z d v+v \int A^{\sigma} f A^{\sigma}\left(\widetilde{\Delta}_{t} f\right) d z d v
\end{aligned}
$$

where, as before, CK stands for "Cauchy-Kovalevskaya",

$$
\mathrm{CK}_{w}=\sum_{k} \int \frac{\partial_{t} w_{k}(t, \eta)}{w_{k}(t, \eta)}\left|A_{k}^{\sigma}(t, \eta) \hat{f}_{k}(t, \eta)\right|^{2} d \eta
$$

To treat the second term in (2.40), we have

$$
\begin{aligned}
\int A^{\sigma} f A^{\sigma}\left(u \nabla_{z, v} f\right) d z d v= & -\frac{1}{2} \int \nabla \cdot u\left|A^{\sigma} f\right|^{2} d v d z \\
& +\int A^{\sigma} f\left[A^{\sigma}(u \cdot \nabla f)-u \cdot \nabla A^{\sigma} f\right] d z d v .
\end{aligned}
$$

Notice that the relative velocity is not divergence-free:

$$
\nabla \cdot u=\partial_{v} g+\partial_{z} \phi \partial_{v} v^{\prime}=\partial_{v} g+\partial_{z} P_{\neq} \phi \partial_{v} h
$$

The first term is controlled by the bootstrap hypothesis (2.25). For the second term we use the elliptic estimates, Lemma 4.1, which show that under the bootstrap hypotheses we have

$$
\left\|P_{\neq} \phi\right\|_{H^{\sigma-4}} \lesssim \frac{\varepsilon v^{\frac{1}{3}}}{\langle t\rangle^{2}} .
$$


Therefore, by the Sobolev embedding, $\sigma>40$ and the bootstrap hypotheses,

$$
\begin{aligned}
\left.\left|\int \nabla \cdot u\right| A^{\sigma} f\right|^{2} d v d z \mid & \lesssim\|\nabla u\|_{L^{\infty}}\left\|A^{\sigma} f\right\|_{2}^{2} \\
& \lesssim\left(\|g\|_{H^{2}}+\left(1+\|h\|_{H^{2}}\right)\left\|P_{\neq} \phi\right\|_{H^{3}}\right)\left\|A^{\sigma} f\right\|_{2}^{2} \\
& \lesssim \frac{\varepsilon v^{\frac{1}{3}}}{\langle t\rangle^{2}}\left\|A^{\sigma} f\right\|_{2}^{2} .
\end{aligned}
$$

To handle the commutator, $\int A^{\sigma} f\left[A^{\sigma}(u \cdot \nabla f)-u \cdot \nabla A^{\sigma} f\right] d z d v$, we use a paraproduct decomposition. Precisely, we define the three main contributors: transport, reaction and remainder:

$$
\begin{aligned}
& \int A^{\sigma} f\left[A^{\sigma}(u \cdot \nabla f)-u \cdot \nabla A^{\sigma} f\right] d z d v \\
& =\frac{1}{2 \pi} \sum_{N \geq 8} T_{N}+\frac{1}{2 \pi} \sum_{N \geq 8} R_{N}+\frac{1}{2 \pi} \mathcal{R}
\end{aligned}
$$

where

$$
\begin{aligned}
T_{N} & =2 \pi \int A^{\sigma} f\left[A^{\sigma}\left(u_{<N / 8} \cdot \nabla f_{N}\right)-u_{<N / 8} \cdot \nabla A^{\sigma} f_{N}\right] d z d v \\
R_{N} & =2 \pi \int A^{\sigma} f\left[A^{\sigma}\left(u_{N} \cdot \nabla f_{<N / 8}\right)-u_{N} \cdot \nabla A^{\sigma} f_{<N / 8}\right] d z d v \\
\mathcal{R} & =2 \pi \sum_{N \in \mathbb{D}} \sum_{\frac{1}{8} N \leq N^{\prime} \leq 8 N} \int A^{\sigma} f\left[A^{\sigma}\left(u_{N} \cdot \nabla f_{N^{\prime}}\right)-u_{N} \cdot \nabla A^{\sigma} f_{N^{\prime}}\right] d z d v .
\end{aligned}
$$

Here $N \in \mathbb{D}=\left\{\frac{1}{2}, 1,2,4, \ldots, 2^{j}, \ldots\right\}, g_{N}$ denotes the $N$ th Littlewood-Paley projection and $g_{<N}$ means the Littlewood-Paley projection onto frequencies less than $N$.

For the last term, we get

$$
\begin{aligned}
v \int A^{\sigma} f A^{\sigma}\left(\widetilde{\Delta}_{t} f\right) d z d v= & v \int A^{\sigma} f A^{\sigma}\left(\Delta_{L} f\right) d z d v \\
& -v \int A^{\sigma} f A^{\sigma}\left(\left(1-\left(v^{\prime}\right)^{2}\right)\left(\partial_{v}-t \partial_{z}\right)^{2} f\right) d z d v \\
= & -v\left\|\sqrt{-\Delta_{L}} A^{\sigma} f\right\|_{2}^{2} \\
& -v \int A^{\sigma} f_{\neq} A^{\sigma}\left(\left(1-\left(v^{\prime}\right)^{2}\right)\left(\partial_{v}-t \partial_{z}\right)^{2} f_{\neq}\right) d z d v \\
& -v \int A^{\sigma} f_{0} A^{\sigma}\left(\left(1-\left(v^{\prime}\right)^{2}\right) \partial_{v}^{2} f_{0}\right) d v \\
= & -v\left\|\sqrt{-\Delta_{L}} A^{\sigma} f\right\|_{2}^{2}+E^{\neq}+E^{0} .
\end{aligned}
$$

The next four propositions, together with (2.43), imply (2.30). First, we deal with the dissipation term. In Section 5 we will prove the following proposition. 
Proposition 2.5. Under the bootstrap hypotheses,

$v \int_{1}^{t}\left(\int A^{\sigma} f A^{\sigma}\left(\tilde{\Delta}_{t} f\right) d z d v\right) d t^{\prime} \leq-\frac{7}{8} v \int_{1}^{t}\left\|\sqrt{-\Delta_{L}} A^{\sigma} f\left(t^{\prime}\right)\right\|_{2}^{2} d t^{\prime}+C \varepsilon^{3} v^{\frac{2}{3}}$.

Next we control the transport part. In Section 6 we will prove the following proposition.

Proposition 2.6. Under the bootstrap hypotheses,

$$
\int_{1}^{t} \sum_{N \geq 8}\left|T_{N}\left(t^{\prime}\right)\right| d t^{\prime} \lesssim \varepsilon \sup _{t^{\prime} \in[1, t]}\left\|A^{\sigma} f\left(t^{\prime}\right)\right\|_{2}^{2} .
$$

Next we control the remainder. In Section 7 we will prove the following proposition.

Proposition 2.7. Under the bootstrap hypotheses,

$$
|\mathcal{R}(t)| \lesssim \frac{\varepsilon v^{\frac{1}{3}}}{\langle t\rangle^{2}}\left\|A^{\sigma} f\right\|_{2}^{2} .
$$

Finally, we control the reaction part. In Section 8 we will prove the following proposition.

Proposition 2.8. Under the bootstrap hypotheses,

$$
\int_{1}^{t} \sum_{N \geq 8}\left|R_{N}\left(t^{\prime}\right)\right| d t^{\prime} \lesssim \varepsilon \sup _{t^{\prime} \in[1, t]}\left\|A^{\sigma} f\left(t^{\prime}\right)\right\|_{2}^{2}+\varepsilon \int_{1}^{t} \mathrm{CK}_{w}\left(t^{\prime}\right) d t^{\prime}+\varepsilon^{3} v^{\frac{2}{3}} .
$$

Let us admit the above propositions and finish the proof of (2.30).

Proof of (2.30). We then get by (2.40) that

$$
\begin{aligned}
\left\|A^{\sigma} f(t)\right\|_{2}^{2}+2 \int_{1}^{t} \mathrm{CK}_{w}\left(t^{\prime}\right) d t^{\prime} \\
=\left\|A^{\sigma} f(1)\right\|_{2}^{2}-2 \int_{1}^{t} \int A^{\sigma} f A^{\sigma}(u \nabla f) d z d v d t^{\prime} \\
\quad+v 2 \int_{1}^{t} \int A^{\sigma} f A^{\sigma}\left(\widetilde{\Delta}_{t} f\right) d z d v d t^{\prime} \\
\leq\left\|A^{\sigma} f(1)\right\|_{2}^{2}-\frac{7}{4} v \int_{1}^{t}\left\|\sqrt{-\Delta_{L}} A^{\sigma} f\left(t^{\prime}\right)\right\|_{2}^{2} d t^{\prime}+C \varepsilon^{3} v^{\frac{2}{3}} \\
\quad+C \int_{1}^{t}\left[\left.\left|\int \nabla \cdot u\right| A^{\sigma} f\right|^{2} d v d z\left|+\sum_{N \geq 8}\right| T_{N}\left(t^{\prime}\right)|+| \mathcal{R}\left(t^{\prime}\right)\left|+\sum_{N \geq 8}\right| R_{N}\left(t^{\prime}\right) \mid\right] d t^{\prime} .
\end{aligned}
$$

Thus, by (2.43) and the above propositions we have

$$
\begin{aligned}
& \left\|A^{\sigma} f(t)\right\|_{2}^{2}+2 \int_{1}^{t} \mathrm{CK}_{w}\left(t^{\prime}\right) d t^{\prime}+\frac{7}{4} v \int_{1}^{t}\left\|\sqrt{-\Delta_{L}} A^{\sigma} f\left(t^{\prime}\right)\right\|_{2}^{2} d t^{\prime} \\
& \quad \leq\left\|A^{\sigma} f(1)\right\|_{2}^{2}+C \varepsilon^{3} v^{\frac{2}{3}}+C \varepsilon \sup _{t^{\prime} \in[1, t]}\left\|A^{\sigma} f\left(t^{\prime}\right)\right\|_{2}^{2}+C \varepsilon \int_{1}^{t} \mathrm{CK}_{w}\left(t^{\prime}\right) d t^{\prime} .
\end{aligned}
$$

Thus, by taking $\varepsilon$ small enough we have proved (2.30). 


\section{Toy model and nonlinear growth}

In this section we study nonlinear growth. For simplicity of notation, in this section we usually take $\eta, k>0$ but the work applies equally well to $\eta, k<0$. Note that modes where $\eta k<0$ do not have resonance for positive times.

\subsection{The toy model}

According to the change of coordinates, the relative velocity now is time integrable. The growth may come from the reaction term. In each time interval $I_{m, \eta}$ which contains only one Orr critical time $t=\frac{\eta}{m}$, it is necessary to study the following toy model:

$$
\begin{aligned}
& \partial_{t} \hat{f}(t, m, \eta)+v\left(k^{2}+(\eta-m t)^{2}\right) \hat{f}(t, m, \eta) \\
& \quad=\int_{|\eta-\xi| \leq 1} \sum_{m-l= \pm 1} \frac{ \pm \xi}{l^{2}+(\xi-l t)^{2}} \hat{f}(t, l, \xi) \hat{f}(t, \pm 1, \eta-\xi) d \xi, \\
& \partial_{t} \hat{f}(t, m \pm 1, \eta)+v\left((m \pm 1)^{2}+(\eta-(m \pm 1) t)^{2}\right) \hat{f}(t, m, \eta) \\
& \quad=\int_{|\eta-\xi| \leq 1} \frac{\xi}{m^{2}+(\xi-m t)^{2}} \hat{f}(t, m, \xi) \hat{f}(t, \pm 1, \eta-\xi) d \xi .
\end{aligned}
$$

Since the $\hat{f}(t, \pm 1, \xi-\eta)$ is restricted to the lower frequency $|\xi-\eta| \leq 1$, we can regard it as a constant in the $\xi$ variable. Moreover, $\hat{f}(t, \pm 1, \xi-\eta)$ also has enhanced dissipation. As $t \in I_{m, \eta},(m \pm 1)^{2}+(\eta-(m \pm 1) t)^{2} \approx \frac{\eta^{2}}{m^{2}}$, thus we deduce the following simplified toy model:

$$
\begin{aligned}
\partial_{t} \hat{f}(t, m, \eta)+v\left(m^{2}+(\eta-m t)^{2}\right) \hat{f}(t, m, \eta) & =\frac{\kappa e^{-c v^{\frac{1}{3}} t} m^{2}}{|\eta|} \hat{f}(t, m \pm 1, \eta) \\
\partial_{t} \hat{f}(t, m \pm 1, \eta)+\frac{v \eta^{2}}{m^{2}} \hat{f}(t, m \pm 1, \eta) & =\frac{\kappa|\eta| e^{-c v^{\frac{1}{3}} t}}{m^{2}\left(1+\left(\frac{\eta}{m}-t\right)^{2}\right)} \hat{f}(t, m, \eta)
\end{aligned}
$$

where $\kappa$ stands for the smallness assumption of the initial data. Our goal is to find the largest $\kappa$ such that we can control the total growth caused by the toy model. Thus we assume the enhanced dissipation is $e^{-c v^{\frac{1}{3}} t}$.

The next step of the simplification is based on the following observations:

- When $t \gg v^{-\frac{1}{3}}$, the enhanced dissipation will offer a small coefficient which makes the Orr mechanism weaker. So we focus on the region of time $t \lesssim v^{-\frac{1}{3}}$. The resonant time region is $I_{t}(\eta) \approx[\sqrt{|\eta|}, 2|\eta|]$. We are interested in the case $|\eta| \lesssim v^{-\frac{2}{3}}$ so that $I_{t}(\eta) \cap\left[1, C v^{-\frac{1}{3}}\right] \neq \emptyset$. During this region of time, $e^{-c v^{\frac{1}{3}} t} \approx 1$.

- The rapid growth of $\hat{f}(t, m \pm 1, \eta)$ happens when $\left|t-\frac{\eta}{m}\right| \approx 1$.

- The coefficient in front of $\hat{f}(t, m, \eta)$ on the right-hand side of (3.2) is much bigger than the coefficient in front of $\hat{f}(t, m \pm 1, \eta)$ on the right-hand side of (3.1). It means that $\hat{f}(t, m \pm 1, \eta)$ will grow faster than $\hat{f}(t, m, \eta)$. We may replace $\hat{f}(t, m, \eta)$ by $\hat{f}(t, m \pm 1, \eta)$ in the second equation. 
- Since $|\eta| \lesssim v^{-\frac{2}{3}}$, when $\left|t-\frac{\eta}{m}\right| \approx 1$, the dissipation coefficient in (3.2), $\frac{v \eta^{2}}{m^{2}} \lesssim \frac{v^{\frac{1}{3}} \eta}{m^{2}}$, is not bigger than the coefficient of the right-hand side if $\kappa \approx v^{\frac{1}{3}}$. Thus we can remove the dissipation term.

Thus we deduce to the following toy model:

$$
\partial_{t} \hat{f}(t, m \pm 1, \eta)=\frac{\kappa|\eta| e^{-c v^{\frac{1}{3}} t}}{m^{2}\left(1+\left(\frac{\eta}{m}-t\right)^{2}\right)} \hat{f}(t, m \pm 1, \eta) .
$$

For $t \in I_{m, \eta}$, let $\tau=t-\frac{\eta}{m}$; then $\tau \in\left[-D_{m, \eta}^{-}, D_{m, \eta}^{+}\right]$, where $D_{m, \eta}^{-}=\frac{\eta}{(2 m+1) m}=$ $\frac{\eta}{m}-t_{m, \eta}$ and $D_{m, \eta}^{+}=\frac{\eta}{(2 m-1) m}=t_{m-1, \eta}-\frac{\eta}{m}$ for $m \geq 1$; then $D_{m, \eta}^{ \pm} \approx \frac{\eta}{m^{2}}$.

Finally, we use the following model to control the entropy growth in each critical time region:

$$
\left\{\begin{array}{l}
\partial_{\tau} g_{m}=\left\langle v^{\frac{1}{3}} t_{m, \eta}\right\rangle^{-(1+\beta)} \frac{v^{\frac{1}{3}} \frac{\eta}{m^{2}}}{1+\tau^{2}} g_{m}, \\
g_{m}\left(-D_{m, \eta}^{-}\right)=1
\end{array}\right.
$$

We need to point out that in the toy model, $e^{-c v^{\frac{1}{3}} t}$ is replaced by $\left\langle v^{\frac{1}{3}} t_{m, \eta}\right\rangle^{-(1+\beta)}$, with $0<\beta \leq \frac{1}{2}$, owing to some technical reasons when we deal with the zero mode (see (8.6)). The condition $\beta>0$ ensures the total growth is bounded (see Lemma 3.3).

For $m \eta>0$ and $|m| \in[1, E(\sqrt{|\eta|})]$, with $|\eta| \geq 3$, we define for $0<\beta \leq \frac{1}{2}$,

$$
g_{m}(\tau, \eta)=\exp \left(\left\langle v^{\frac{1}{3}} t_{m, \eta}\right\rangle^{-(1+\beta)} \frac{\nu^{\frac{1}{3}} \eta}{m^{2}}\left(\arctan (\tau)+\arctan \left(D_{m, \eta}^{-}\right)\right)\right)
$$

then $g_{m}$ solves (3.3).

Then we have

$$
g_{m}\left(D_{m, \eta}^{+}, \eta\right)=G_{m}(\eta) \underbrace{g_{m}\left(-D_{m, \eta}^{-}, \eta\right)}_{=1},
$$

with

$$
G_{m}(\eta)=\exp \left(\left\langle v^{\frac{1}{3}} t_{m, \eta}\right\rangle^{-(1+\beta)} \frac{v^{\frac{1}{3}} \eta}{m^{2}}\left(\arctan \left(D_{m, \eta}^{+}\right)+\arctan \left(D_{m, \eta}^{-}\right)\right)\right) .
$$

Otherwise, we let $g_{m}(\tau, \eta)=1$.

\subsection{Key multiplier}

In this subsection we will define the key multiplier that governs the growth.

We define $w(t, \eta)$ in the following way:

- for $t \leq t(\eta), w(t, \eta)=1$;

- for $t \in I_{j, \eta}$ with $|j| \in[1, E(\sqrt{|\eta|})]$ and $j \eta>0$, we have $w(t, \eta)=w\left(t_{j, \eta}, \eta\right) \times$ $g_{j}\left(t-\frac{\eta}{j}, \eta\right)$

- $\quad$ for $t \geq 2|\eta|$, we have $w(t, \eta)=w(2|\eta|, \eta)$. 
According to the definition of $g_{m}$, we get

$$
\frac{\partial_{t} w(t, \eta)}{w(t, \eta)} \approx \frac{\left\langle v^{\frac{1}{3}} t\right\rangle^{-(1+\beta)} v^{\frac{1}{3}} \frac{\eta}{m^{2}}}{1+\left(t-\frac{\eta}{m}\right)^{2}} 1_{t \in I_{m, \eta}} \approx \frac{\left\langle v^{\frac{1}{3}} t\right\rangle^{-\beta} m^{-1}}{1+\left(t-\frac{\eta}{m}\right)^{2}} 1_{t \in I_{m, \eta}} .
$$

Next, for $m \eta>0$ and $|m| \in[1, E(\sqrt{|\eta|})]$, with $|\eta| \geq 3$, we will construct a continuous function $\varrho(m, \eta) \approx \frac{m}{|m|} \max \{|m|,|\eta|\}$. First, let $\rho(x)$ be a bounded smooth function such that

$$
\rho(x)= \begin{cases}1, & x \geq \frac{1}{10}, \\ \text { smooth connected, } & x \in\left[\frac{1}{20}, \frac{1}{10}\right], \\ 0, & x \leq \frac{1}{20} .\end{cases}
$$

We also let $\rho$ satisfy

$$
\int_{\frac{1}{20}}^{\frac{1}{10}} \rho(x) d x=\frac{1}{20} .
$$

Let $\rho_{k}(x)=\rho\left(\frac{x}{|k|}\right)$ and for $k \eta \geq 0$,

$$
w_{k}(t, \eta)=w(t, \varrho(k, \eta))
$$

where

$$
\varrho(k, \eta)= \begin{cases}\frac{|k|}{20}+\int_{0}^{|\eta|} \rho_{k}(x) d x, & k \neq 0, \\ |\eta|, & k=0 .\end{cases}
$$

Then we get that for $|\eta| \leq \frac{|k|}{20}$, we have $\varrho(k, \eta)=\frac{|k|}{20}$ and $w_{k}(t, \eta)=w\left(t, \frac{k}{20}\right)$, and for $|\eta| \geq \frac{|k|}{10}$, we have $\varrho(k, \eta)=|\eta|$ and $w_{k}(t, \eta)=w(t, \eta)$.

Lemma 3.1. It holds that

$$
\varrho(k, \eta) \approx\langle k, \eta\rangle .
$$

For $|k-l, \xi-\eta| \leq \frac{1}{100}|l, \xi|$, it holds that

$$
|\varrho(k, \eta)-\varrho(l, \xi)| \lesssim|k-l, \xi-\eta| .
$$

Proof. It is easy to obtain that $\varrho(k, \eta) \lesssim\langle k, \eta\rangle$. The lower bound follows from the fact that for $\frac{|k|}{20} \leq|\eta| \leq \frac{|k|}{10}$, we have $\varrho(k, \eta) \gtrsim \frac{|k|}{20} \geq \frac{|\eta|}{2}$.

If $|\xi| \geq|l|$, then

$$
|k-l, \xi-\eta| \leq \frac{1}{50}|\xi|, \quad|\eta| \geq \frac{49}{50}|\xi| \quad \text { and } \quad|k| \leq|k-l|+|l| \leq \frac{51}{50}|\xi| \leq 2|\eta| .
$$

Thus,

$$
|\varrho(k, \eta)-\varrho(l, \xi)|=|\xi-\eta|
$$

If $|\xi| \leq \frac{|l|}{100}$, then

$|k-l, \xi-\eta| \leq \frac{101}{10000}|l|, \quad|k| \geq \frac{9899}{10000}|l| \quad$ and $\quad|\eta| \leq|\xi|+|\xi-\eta| \leq \frac{201}{10000}|l| \leq \frac{|k|}{20}$ 
Thus,

$$
|\varrho(k, \eta)-\varrho(l, \xi)|=\frac{1}{20}|k-l| .
$$

Then we only need to focus on $|\xi| \approx|l| \approx|\eta| \approx|k|$. Thus,

$$
\begin{aligned}
|\varrho(k, \eta)-\varrho(l, \xi)| & =\left|\frac{|k|}{20}-\frac{|l|}{20}+\right| k\left|\int_{0}^{\frac{\eta}{k}} \rho(x) d x-\right| l\left|\int_{0}^{\frac{\xi}{l}} \rho(x) d x\right| \\
& \lesssim|k-l|+|k-l| \int_{0}^{\frac{\eta}{k}} \rho(x) d x+\left|l \int_{\frac{\eta}{k}}^{\frac{\xi}{l}} \rho(x) d x\right| \\
& \lesssim|k-l|+\frac{|\eta l-\xi k|}{|k|} \lesssim|k-l|+\frac{|\eta, k||k-l, \eta-\xi|}{|k|} \\
& \lesssim|k-l, \xi-\eta| .
\end{aligned}
$$

Thus we have proved the lemma.

With $w_{k}(t, \eta)$, we can now define our key multiplier $A_{k}^{\sigma}(t, \eta)$,

$$
A_{k}^{\sigma}(t, \eta)=\frac{\langle k, \eta\rangle^{\sigma}}{w_{k}(t, \eta)} .
$$

\subsection{Basic estimate for the multiplier}

The following lemma expresses that the critical times are well separated.

Lemma 3.2 ([7]). Let $\xi$, $\eta$ be such that there exists some $\alpha \geq 1$ with $\alpha^{-1}|\xi| \leq|\eta| \leq \alpha|\xi|$ and let $k, n$ be such that $t \in I_{k, \eta} \cap I_{n, \xi}$; then $k \approx n$ and, moreover, at least one of the following holds:

(a) $k=n$;

(b) $\left|t-\frac{\eta}{k}\right| \geq \frac{1}{10 \alpha} \frac{\eta}{k^{2}}$ and $\left|t-\frac{\xi}{n}\right| \geq \frac{1}{10 \alpha} \frac{\xi}{n^{2}}$;

(c) $|\eta-\xi| \gtrsim \alpha \frac{|\eta|}{|n|}$.

Now we will present a lemma about the upper and lower bound estimates for $w(t, \eta)$.

Lemma 3.3. It holds that

$$
w(t, \eta) \approx 1 .
$$

As a consequence, $A_{k}^{\sigma}(t, \eta) \approx\langle k, \eta\rangle^{\sigma}$.

Proof. We have for any $t, \eta$,

$$
\begin{aligned}
1 & \leq w(t, \eta) \leq \prod_{m=E(\sqrt{|\eta|})}^{1} G_{m}(\eta) \\
& \leq \exp \left(\sum_{m=E(\sqrt{|\eta|})}^{1} \frac{\pi v^{\frac{1}{3}} \eta}{m^{2}}\left(1+v^{\frac{1}{3}} t_{m, \eta}\right)^{-(1+\beta)}\right)
\end{aligned}
$$




$$
\begin{aligned}
& \lesssim \begin{cases}\exp \left(C \sum_{m=E(\sqrt{|\eta|})}^{1} \frac{v^{\frac{1}{3}} \eta}{m^{2}}\right), & v^{\frac{1}{3}} \eta \leq 1, \\
\exp \left(C \sum_{m=v^{\frac{1}{3}} \eta}^{E(\sqrt{|\eta|})} \frac{v^{\frac{1}{3}} \eta}{m^{2}}+C \sum_{m=v^{\frac{1}{3}} \eta}^{1} \frac{m^{-1+\beta}}{\left(v^{\frac{1}{3}} \eta\right)^{\beta}}\right), & 1 \leq v^{\frac{1}{3}} \eta \leq E(\sqrt{|\eta|}), \\
\exp \left(C \sum_{m=E(\sqrt{|\eta|})}^{1} \frac{m^{-1+\beta}}{\left(v^{\frac{1}{3}} \eta\right)^{\beta}}\right), & 1 \leq E(\sqrt{|\eta|}) \leq v^{\frac{1}{3}} \eta,\end{cases} \\
& \lesssim \begin{cases}v^{\frac{1}{3}} \eta \lesssim 1, & |\eta| \lesssim v^{-\frac{1}{3}}, \\
1, & v^{-\frac{1}{3}} \lesssim|\eta| \lesssim v^{-\frac{2}{3}}, \\
\frac{1}{\left(v^{\frac{1}{3}} \sqrt{|\eta|}\right)^{\beta}} \lesssim 1, & v^{-\frac{2}{3}} \lesssim|\eta| .\end{cases}
\end{aligned}
$$

Thus we have proved the lemma.

The above lemma gives that for all $t$,

$$
A_{k}^{\sigma}(t, \eta) \approx\langle k, \eta\rangle^{\sigma}
$$

Next we introduce several lemmas related to the properties of $D$. The first lemma can be found in [9], which will be useful in the proof of the commutator estimate in Section 10.

Lemma 3.4 ([9]). Uniformly in $v, \eta, \xi$ and $t \geq 1$ we have

$$
D(t, \eta) \gtrsim v \max \left\{|\eta|^{3}, t^{3}\right\}
$$

and

$$
\frac{D(t, \xi)}{D(t, \eta)} \lesssim\langle\eta-\xi\rangle^{3}, \quad|D(t, \xi)-D(t, \eta)| \lesssim \frac{D(t, \xi)}{\langle\xi\rangle+\langle\eta\rangle}\langle\eta-\xi\rangle^{3} .
$$

In the next lemma we will introduce the product lemma related to $D$ which is a Sobolev-type estimate comparable to [9, Lemma 3.7].

Lemma 3.5. The following holds for all $q^{1}$ and $q^{2}$ and $\gamma>1$ :

$$
\left\|D\left(q^{1} q^{2}\right)\right\|_{H^{\gamma}} \lesssim\left\|q^{1}\right\|_{H^{\gamma+3}}\left\|D q^{2}\right\|_{H^{\gamma}}
$$

and

$$
\left\|D\left(\nabla^{\perp} q^{1} \cdot \nabla q^{2}\right)\right\|_{H^{\gamma}} \lesssim\left\|q^{1}\right\|_{H^{\gamma+5}}\left\|D q^{2}\right\|_{H^{\gamma}}+\left\|D q_{1}\right\|_{H^{\gamma}}\left\|q_{2}\right\|_{H^{\gamma+5}} .
$$

Proof. We use the dual method. By Lemma 3.4, we get

$$
\begin{aligned}
\left\|D\left(q^{1} q^{2}\right)\right\|_{H^{\gamma}} & =\left\|\langle\nabla\rangle^{\gamma} D\left(q^{1} q^{2}\right)\right\|_{L^{2}} \\
& =\sup _{\|\varphi\|_{L^{2}}=1}\left|\sum_{k, l} \int_{\eta, \xi} \hat{\varphi}_{k}(\eta)\langle k, \eta\rangle^{\gamma} D(\eta) \hat{q}_{k-l}^{1}(\eta-\xi) \hat{q}_{l}^{2}(\xi) d \xi d \eta\right|
\end{aligned}
$$




$$
\begin{aligned}
& \lesssim \sup _{\|\varphi\|_{L^{2}}=1} \sum_{k, l} \int_{\eta, \xi}\left|\hat{\varphi}_{k}(\eta)\right|\langle k, \eta\rangle^{\gamma}\langle\xi-\eta\rangle^{3}\left|\hat{q}_{k-l}^{1}(\eta-\xi)\right|\left|D(\xi) \hat{q}_{l}^{2}(\xi)\right| d \xi d \eta \\
& \lesssim \sup _{\|\varphi\|_{L^{2}}=1} \sum_{k, l} \int_{\eta, \xi} 1_{\substack{|k-l, \eta-\xi| \\
\leq|l, \xi|}}\left|\hat{\varphi}_{k}(\eta)\right|\langle\xi-\eta\rangle^{3}\left|\hat{q}_{k-l}^{1}(\eta-\xi)\right|\left|D(\xi)\langle l, \xi\rangle^{\gamma} \hat{q}_{l}^{2}(\xi)\right| d \xi d \eta \\
& +\sup _{\|\varphi\|_{L^{2}}=1} \sum_{k, l} \int_{\eta, \xi} 1_{\substack{|k-l, \eta-\xi \xi| \\
>|l, \xi|}}\left|\hat{\varphi}_{k}(\eta)\right|\langle k-l, \eta-\xi\rangle^{\gamma}\langle\xi-\eta\rangle^{3}\left|\hat{q}_{k-l}^{1}(\eta-\xi)\right| \\
& \times \| D(\xi) \hat{q}_{l}^{2}(\xi) \mid d \xi d \eta \\
& \therefore\|\varphi\|_{L^{2}}\left\|D q^{2}\right\|_{H^{\gamma}}\left\|q^{1}\right\|_{H^{\gamma+3}} .
\end{aligned}
$$

In the last inequality we use the fact that $\|\hat{q}\|_{L^{1}} \lesssim\left\|\langle k, \eta\rangle^{\gamma} \hat{q}\right\|_{L^{2}}\left\|\langle k, \eta\rangle^{-\gamma}\right\|_{L^{2}} \lesssim\|q\|_{H^{\gamma}}$ for $\gamma>1$.

We also have

$$
\begin{aligned}
& \left\|D\left(\nabla^{\perp} q^{1} \cdot \nabla q^{2}\right)\right\|_{H^{\gamma}} \\
& =\sup _{\|\varphi\|_{L^{2}}=1}\left|\sum_{k, l} \int_{\eta, \xi} \hat{\varphi}_{k}(\eta)\langle k, \eta\rangle^{\gamma} D(\eta) \hat{q}_{k-l}^{1}(\eta-\xi) \hat{q}_{l}^{2}(\xi)(-\eta+\xi, k-l) \cdot(l, \xi) d \xi d \eta\right| \\
& \lesssim \sup _{\|\varphi\|_{L^{2}}=1} \sum_{k, l} \int_{\eta, \xi} 1_{\substack{|k-l, \eta-\xi| \\
\leq|l, \xi|}}\left|\hat{\varphi}_{k}(\eta)\right|\left|D(\eta-\xi) \hat{q}_{k-l}^{1}(\eta-\xi)\right|\left|\langle\xi\rangle^{3}\langle l, \xi\rangle^{\gamma+2} \hat{q}_{l}^{2}(\xi)\right| d \xi d \eta \\
& +\sup _{\|\varphi\|_{L^{2}}=1} \sum_{k, l} \int_{\eta, \xi} 1_{\substack{|k-l, \eta-\xi| \\
>|l, \xi|}}\left|\hat{\varphi}_{k}(\eta)\right|\langle k-l, \eta-\xi\rangle^{\gamma+2}\langle\xi-\eta\rangle^{3}\left|\hat{q}_{k-l}^{1}(\eta-\xi)\right| \\
& \times\left|D(\xi) \hat{q}_{l}^{2}(\xi)\right| d \xi d \eta \\
& \lesssim\|\varphi\|_{L^{2}}\left\|D q^{2}\right\|_{H^{\gamma}}\left\|q^{1}\right\|_{H^{\gamma+5}}+\|\varphi\|_{L^{2}}\left\|D q^{1}\right\|_{H^{\gamma}}\left\|q^{2}\right\|_{H^{\gamma+5}} \text {. }
\end{aligned}
$$

Thus we have proved the lemma.

\section{Elliptic estimate}

The purpose of this section is to provide a thorough analysis of $\Delta_{t}$.

Lemma 4.1. Under the bootstrap hypotheses, for $v$ sufficiently small and $s^{\prime} \in[0,2]$, it holds that for $2 \leq \gamma \leq \sigma-1$,

$$
\left\|P_{\neq} \phi\right\|_{H^{\gamma-s^{\prime}}} \lesssim \frac{1}{\langle t\rangle^{s^{\prime}}}\left\|\left\langle\partial_{z}\right\rangle^{-s^{\prime}} f_{\neq}\right\|_{H^{\gamma}},
$$

and for $\gamma \leq \sigma-1$,

$$
\left\|\Delta_{L} \Delta_{t}^{-1} P_{\neq} f\right\|_{H^{\gamma}}=\left\|\Delta_{L} P_{\neq} \phi\right\|_{H^{\gamma}} \lesssim\left\|P_{\neq} f\right\|_{H^{\gamma}} .
$$


Proof. For $s^{\prime} \in[0,2]$ and $s \geq 0$ we get

$$
\begin{aligned}
\left\|P_{\neq} \phi\right\|_{H^{s}}^{2} & =\sum_{k \neq 0} \int_{\eta}\langle k, \eta\rangle^{2 s}|\widehat{\phi}(k, \eta)|^{2} d \eta \\
& \leq \sum_{k \neq 0} \int_{\eta} \frac{\langle k, \eta\rangle^{2 s}\left\langle\frac{\eta}{k}\right\rangle^{2 s^{\prime}}}{\left\langle\frac{\eta}{k}\right\rangle^{2 s^{\prime}}\left(k^{2}+(\eta-k t)^{2}\right)^{2}}\left|\widehat{\Delta_{L} \phi}(k, \eta)\right|^{2} d \eta \\
& \lesssim \sum_{k \neq 0} \int_{\eta} \frac{\langle k, \eta\rangle^{2 s+2 s^{\prime}}}{k^{2 s^{\prime}}\left(1+t^{2}\right)^{s^{\prime}}}\left|\widehat{\Delta_{L} \phi}(k, \eta)\right|^{2} d \eta \\
& \lesssim \frac{1}{\left(1+t^{2}\right)^{s^{\prime}}}\left\|\left\langle\partial_{z}\right\rangle^{-s^{\prime}} \Delta_{L} P_{\neq} \phi\right\|_{H^{s+s^{\prime}}}^{2} .
\end{aligned}
$$

We write $\Delta_{t}$ as a perturbation of $\Delta_{L}$ via

$$
\Delta_{L} P_{\neq} \phi=P_{\neq} f+\left(1-\left(v^{\prime}\right)^{2}\right)\left(\partial_{v}-t \partial_{z}\right)^{2} P_{\neq} \phi-v^{\prime \prime}\left(\partial_{v}-t \partial_{z}\right) P_{\neq} \phi
$$

Thus we get

$$
\begin{aligned}
\left\|\Delta_{L} P_{\neq} \phi\right\|_{H^{\gamma}} \leq & \left\|P_{\neq} f\right\|_{H^{\gamma}}+C\left\|\left(1-\left(v^{\prime}\right)^{2}\right)\left(\partial_{v}-t \partial_{z}\right)^{2} P_{\neq} \phi\right\|_{H^{\gamma}} \\
& +C\left\|v^{\prime \prime}\left(\partial_{v}-t \partial_{z}\right) P_{\neq} \phi\right\|_{H^{\gamma}}
\end{aligned}
$$

then by using the fact that $v^{\prime \prime}=(h+1) \partial_{v} h,(\mathrm{~A} .3)$ and the bootstrap hypotheses, we get

$$
\begin{aligned}
\left\|\Delta_{L} P_{\neq} \phi\right\|_{H^{\gamma}} \leq & \left\|P_{\neq} f\right\|_{H^{\gamma}}+C\|h\|_{H^{\sigma-1}}\left(1+\|h\|_{\sigma-1}\right)\left\|\Delta_{L} P_{\neq} \phi\right\|_{H^{\gamma}} \\
& +C\left(1+\|h\|_{\sigma-1}\right)\|h\|_{H^{\sigma}}\left\|\Delta_{L} P_{\neq} \phi\right\|_{H^{\gamma}} \\
\lesssim & \left\|P_{\neq} f\right\|_{H^{\gamma}}+C \varepsilon v^{\frac{1}{6}}\left\|\Delta_{L} P_{\neq} \phi\right\|_{H^{\gamma}}
\end{aligned}
$$

which implies $\left\|\Delta_{L} P_{\neq} \phi\right\|_{H^{\gamma}} \lesssim\left\|P_{\neq} f\right\|_{H^{\gamma}}$. The lemma follows from (4.1) with $s=$ $\sigma-2-s^{\prime}$.

As $\left(1-\left(v^{\prime}\right)^{2}\right)$ and $v^{\prime \prime}$ are the zero mode, by the same argument as the proof we can easily get that for $\gamma \leq \sigma-1$,

$$
\left\|\left\langle\partial_{z}\right\rangle^{\sigma-\gamma}\left\langle\partial_{v}\right\rangle^{\gamma} \Delta_{L} \Delta_{t}^{-1} f_{\neq}\right\|_{2} \lesssim\left\|f_{\neq}\right\|_{H^{\sigma}} \lesssim\left\|A^{\sigma} f\right\|_{2} .
$$

Lemma 4.2. Under the bootstrap hypotheses, it holds that

$$
\left\|\nabla_{L} P_{\neq} \phi\right\|_{H^{\sigma-2}}+\left\|\tilde{u}_{\neq}\right\|_{H^{\sigma-2}} \lesssim \frac{1}{\langle t\rangle}\left\|f_{\neq}\right\|_{H^{\sigma-1}},
$$

and $\gamma \leq \sigma-1$

$$
\left\|\nabla_{L} \tilde{u}_{\neq}\right\|_{H^{\gamma}} \lesssim\left\|f_{\neq}\right\|_{H^{\gamma}}
$$

Proof. By the definition of $\tilde{u}$ we get

$$
\tilde{u}_{\neq}=-(1+h)\left(\partial_{v}-t \partial_{z}\right) P_{\neq} \phi .
$$


Here we use the same argument as (4.1) and we get

$$
\begin{aligned}
\left\|\left(\partial_{v}-t \partial_{z}\right) P_{\neq} \phi\right\|_{H^{s}}^{2} & =\sum_{k \neq 0} \int_{\eta}\langle k, \eta\rangle^{2 s}|\eta-k t|^{2}|\widehat{\phi}(k, \eta)|^{2} d \eta \\
& \leq \sum_{k \neq 0} \int_{\eta} \frac{\langle k, \eta\rangle^{2 s}\left\langle\frac{\eta}{k}\right\rangle^{2}|\eta-k t|^{2}}{\left\langle\frac{\eta}{k}\right\rangle^{2}\left(k^{2}+(\eta-k t)^{2}\right)^{2}}\left|\widehat{\Delta_{L} \phi}(k, \eta)\right|^{2} d \eta \\
& \leq \sum_{k \neq 0} \int_{\eta} \frac{\langle k, \eta\rangle^{2 s}\left\langle\frac{\eta}{k}\right\rangle^{2}}{\left\langle\frac{\eta}{k}\right\rangle^{2}\left(k^{2}+(\eta-k t)^{2}\right)}\left|\widehat{\Delta_{L} \phi}(k, \eta)\right|^{2} d \eta \\
& \lesssim \sum_{k \neq 0} \int_{\eta} \frac{\langle k, \eta\rangle^{2 s+2}}{k^{2}\left(1+t^{2}\right)}\left|\widehat{\Delta_{L} \phi}(k, \eta)\right|^{2} d \eta \\
& \lesssim \frac{1}{1+t^{2}}\left\|\left\langle\partial_{z}\right\rangle^{-1} \Delta_{L} P_{\neq} \phi\right\|_{H^{s+1}}^{2} .
\end{aligned}
$$

Then by Lemma 4.1 and the bootstrap hypotheses, we have

$$
\begin{aligned}
\left\|\tilde{u}_{\neq}\right\|_{H^{\sigma-2}} & \lesssim\left(1+\|h\|_{H^{\sigma-2}}\right)\left\|\left(\partial_{v}-t \partial_{z}\right) P_{\neq} \phi\right\|_{H^{\sigma-2}} \\
& \lesssim \frac{\left(1+\|h\|_{H^{\sigma-2}}\right)}{\langle t\rangle}\left\|\Delta_{L} P_{\neq} \phi\right\|_{H^{\sigma-1}} \lesssim \frac{1}{\langle t\rangle}\left\|f_{\neq}\right\|_{H^{\sigma-1}}
\end{aligned}
$$

The first inequality follows from (4.3) with $s=\sigma-2$.

We also have

$$
\begin{aligned}
\partial_{z} \tilde{u}_{\neq} & =-(1+h)\left(\partial_{v}-t \partial_{z}\right) \partial_{z} P_{\neq} \phi, \\
\left(\partial_{v}-t \partial_{z}\right) \tilde{u}_{\neq} & =-(1+h)\left(\partial_{v}-t \partial_{z}\right)^{2} P_{\neq} \phi-\partial_{v} h\left(\partial_{v}-t \partial_{z}\right) P_{\neq} \phi .
\end{aligned}
$$

Therefore, by Lemma 4.1 and the bootstrap hypotheses, we get

$$
\left\|\nabla_{L} \tilde{u}_{\neq}\right\|_{H^{\gamma}} \lesssim\left(1+\|h\|_{H^{\sigma-1}}\right)\left\|\Delta_{L} P_{\neq} \phi\right\|_{H^{\gamma}} \lesssim\left\|f_{\neq}\right\|_{H^{\gamma}} .
$$

Thus we have proved the lemma.

Lemma 4.3. Under the bootstrap hypotheses, it holds that

$$
\left\|\nabla_{L} \tilde{u}_{\neq}\right\|_{H^{\sigma}} \lesssim\left\|\Delta_{L} \Delta_{t}^{-1} f_{\neq}\right\|_{H^{\sigma}} \lesssim\left\|f_{\neq}\right\|_{H^{\sigma}}+\frac{\varepsilon v^{\frac{1}{3}}}{\langle t\rangle\left\langle v t^{3}\right\rangle}\left\|\partial_{v} h\right\|_{H^{\sigma}}
$$

and

$$
\left\|\sqrt{\frac{\partial_{t} w}{w}} \chi_{R} \Delta_{L} \Delta_{t}^{-1} f_{\neq}\right\|_{H^{\sigma}} \lesssim\left\|\sqrt{\frac{\partial_{t} w}{w}} f_{\neq}\right\|_{H^{\sigma}}+\frac{\varepsilon^{2} v^{\frac{1}{2}}}{\left\langle v t^{3}\right\rangle} .
$$

Proof. We have

$$
\Delta_{L} \Delta_{t}^{-1} f_{\neq}=\Delta_{L} P_{\neq} \phi=P_{\neq} f+\left(1-\left(v^{\prime}\right)^{2}\right)\left(\partial_{v}-t \partial_{z}\right)^{2} P_{\neq} \phi-v^{\prime \prime}\left(\partial_{v}-t \partial_{z}\right) P_{\neq} \phi .
$$


Thus we get

$$
\begin{aligned}
\left\|\Delta_{L} \Delta_{t}^{-1} f_{\neq}\right\|_{H^{\sigma}} \lesssim & \left\|f_{\neq}\right\|_{H^{\sigma}}+\left\|\left(1-\left(v^{\prime}\right)^{2}\right)\right\|_{H^{3}}\left\|\left(\partial_{v}-t \partial_{z}\right)^{2} P_{\neq} \phi\right\|_{H^{\sigma}} \\
& +\left\|\left(1-\left(v^{\prime}\right)^{2}\right)\right\|_{H^{\sigma}}\left\|\left(\partial_{v}-t \partial_{z}\right)^{2} P_{\neq} \phi\right\|_{H^{3}} \\
& +\left\|(1+h) \partial_{v} h\right\|_{H^{3}}\left\|\nabla_{L} P_{\neq} \phi\right\|_{H^{\sigma}}+\left\|(1+h) \partial_{v} h\right\|_{H^{\sigma}}\left\|\nabla_{L} P_{\neq} \phi\right\|_{H^{3}} \\
\lesssim & \left\|f_{\neq}\right\|_{H^{\sigma}}+\varepsilon v^{\frac{1}{3}}\left\|\Delta_{L} \Delta_{t}^{-1} f_{\neq}\right\|_{H^{\sigma}}+\varepsilon v^{\frac{1}{6}}\left\|f_{\neq}\right\|_{H^{3}}+\varepsilon v^{\frac{1}{6}}\left\|\nabla_{L} P_{\neq} \phi\right\|_{H^{3}} \\
& +\left\|\partial_{v} h\right\|_{H^{\sigma}}\left\|\nabla_{L} P_{\neq} \phi\right\|_{H^{3}} \\
\lesssim & \left\|f_{\neq}\right\|_{H^{\sigma}}+\varepsilon v^{\frac{1}{6}}\left\|\Delta_{L} \Delta_{t}^{-1} f_{\neq}\right\|_{H^{\sigma}}+\langle t\rangle^{-1}\left\|\partial_{v} h\right\|_{H^{\sigma}}\left\|\Delta_{L} P \neq \phi\right\|_{H^{4}} \\
\lesssim & \left\|f_{\neq}\right\|_{H^{\sigma}}+\varepsilon v^{\frac{1}{6}}\left\|\Delta_{L} \Delta_{t}^{-1} f_{\neq}\right\|_{H^{\sigma}}+\frac{v^{\frac{1}{3}}}{\langle t\rangle\left\langle v t^{3}\right\rangle}\left\|\partial_{v} h\right\|_{H^{\sigma}} .
\end{aligned}
$$

We also have

$$
\begin{aligned}
\partial_{z} \tilde{u}_{\neq} & =-(1+h)\left(\partial_{v}-t \partial_{z}\right) \partial_{z} P_{\neq} \phi, \\
\left(\partial_{v}-t \partial_{z}\right) \tilde{u}_{\neq} & =-(1+h)\left(\partial_{v}-t \partial_{z}\right)^{2} P_{\neq} \phi-\partial_{v} h\left(\partial_{v}-t \partial_{z}\right) P_{\neq} \phi .
\end{aligned}
$$

Therefore by Lemma 4.1 and the bootstrap hypotheses, we get

$$
\left\|\nabla_{L} \tilde{u}_{\neq}\right\|_{H^{\sigma}} \lesssim\left(1+\|h\|_{H^{\sigma}}\right)\left\|\Delta_{L} P_{\neq} \phi\right\|_{H^{\sigma}} .
$$

By taking $\varepsilon$ small enough, we get the first inequality.

In what follows we use the shorthand

$$
G(\xi)=\widehat{1-\left(v^{\prime}\right)^{2}}(\xi),
$$

and then

$$
\begin{aligned}
& \sqrt{\frac{\partial_{t} w_{k}(t, \eta)}{w_{k}(t, \eta)}} 1_{t \in I_{k, \eta}} 1_{k \neq 0}\left(k^{2}+(\eta-k t)^{2}\right) \phi_{k}(t, \eta) \\
&=\quad \sqrt{\frac{\partial w_{k}(t, \eta)}{w_{k}(t, \eta)}} 1_{t \in I_{k, \eta}} 1_{k \neq 0} f_{k}(t, \eta) \\
& \quad-\sqrt{\frac{\partial_{t} w_{k}(t, \eta)}{w_{k}(t, \eta)}} 1_{t \in I_{k, \eta}} 1_{k \neq 0} \int_{|\xi| \geq|\eta-\xi|} G(\xi)(\eta-\xi-k t)^{2} \widehat{\phi}_{k}(t, \eta-\xi) d \xi \\
&-\sqrt{\frac{\partial_{t} w_{k}(t, \eta)}{w_{k}(t, \eta)}} 1_{t \in I_{k, \eta}} 1_{k \neq 0} \int_{|\xi| \geq|\eta-\xi|} G(\eta-\xi)(\xi-k t)^{2} \widehat{\phi}_{k}(t, \xi) d \xi \\
&-i \sqrt{\frac{\partial_{t} w_{k}(t, \eta)}{w_{k}(t, \eta)}} 1_{t \in I_{k, \eta}} 1_{k \neq 0} \int_{|\xi| \geq|\eta-\xi|} \widehat{v^{\prime \prime}(\xi)(\eta-\xi-k t) \widehat{\phi}_{k}(t, \eta-\xi) d \xi} \\
&-i \sqrt{\frac{\partial_{t} w_{k}(t, \eta)}{w_{k}(t, \eta)}} 1_{t \in I_{k, \eta}} 1_{k \neq 0} \int_{|\xi| \geq|\eta-\xi|} \widehat{v}^{\prime \prime}(\eta-\xi)(\xi-k t) \widehat{\phi}_{k}(t, \xi) d \xi \\
&= \sqrt{\frac{\partial_{t} w_{k}(t, \eta)}{w_{k}(t, \eta)}} 1_{t \in I_{k, \eta}} 1_{k \neq 0} f_{k}(t, \eta)+E_{H L}^{1}+E_{L H}^{1}+E_{H L}^{2}+E_{L H}^{2} .
\end{aligned}
$$


We have $t \approx t_{k, \eta} \approx \frac{\eta}{k}$ and then

$$
\frac{\partial_{t} w_{k}(t, \eta)}{w_{k}(t, \eta)}=\frac{\left\langle v^{\frac{1}{3}} t_{k, \eta}\right\rangle^{-(1+\beta)} v^{\frac{1}{3}} \frac{\eta}{k^{2}}}{1+\left(\frac{\eta}{k}-t\right)^{2}} \lesssim \frac{1}{k}\left\langle v^{\frac{1}{3}} t\right\rangle^{-(1+\beta)} v^{\frac{1}{3}} t \lesssim \frac{1}{k} .
$$

Thus we get

$$
\begin{aligned}
\left\|E_{H L}^{1}\right\|_{H^{\sigma}} & \lesssim\|G\|_{H^{\sigma}}\left\|\left(\partial_{v}-t \partial_{z}\right)^{2} P_{\neq} \phi\right\|_{H^{4}} \\
& \lesssim\|h\|_{H^{\sigma}}\left(\|h\|_{H^{3}}+1\right)\left\|f_{\neq}\right\|_{H^{4}} \\
& \lesssim \frac{\varepsilon^{2} v^{\frac{1}{2}}}{\left\langle v t^{3}\right\rangle}
\end{aligned}
$$

For $E_{L H}^{1}$ we get

$$
\frac{\partial_{t} w_{k}(t, \eta)}{w_{k}(t, \eta)} \frac{w_{k}(t, \xi)}{\partial_{t} w_{k}(t, \xi)} \approx \frac{1+\left|\frac{\xi}{k}-t\right|^{2}}{1+\left|\frac{\eta}{k}-t\right|^{2}} \lesssim\langle\eta-\xi\rangle^{2}
$$

Then we get

$\left\|E_{L H}^{1}\right\|_{H^{\sigma}}$

$$
\begin{aligned}
& \lesssim\left\|\langle k, \eta\rangle^{\sigma} \sqrt{\frac{\partial_{t} w_{k}(t, \eta)}{w_{k}(t, \eta)}} 1_{t \in I_{k, \eta}} 1_{k \neq 0} \int_{|\xi| \geq|\eta-\xi|} \hat{G}(\eta-\xi)(\xi-k t)^{2} \widehat{\phi}_{k}(t, \xi) d \xi\right\|_{L^{2}} \\
& \lesssim\left\|1_{t \in I_{k, \eta}} 1_{k \neq 0} \int_{|\xi| \geq|\eta-\xi|}\langle\eta-\xi\rangle \widehat{G}(\eta-\xi)(\xi-k t)^{2}\langle k, \xi\rangle^{\sigma} \sqrt{\frac{\partial_{t} w_{k}(t, \xi)}{w_{k}(t, \xi)}} \widehat{\phi}_{k}(t, \xi) d \xi\right\|_{L^{2}} \\
& \lesssim\|G\|_{H^{6}}\left\|\sqrt{\frac{\partial_{t} w}{w}} \chi_{R} \Delta_{L} \Delta_{t}^{-1} f_{\neq}\right\|_{H^{\sigma}} \lesssim \varepsilon v^{\frac{1}{3}}\left\|\sqrt{\frac{\partial_{t} w}{w}} \chi_{R} \Delta_{L} \Delta_{t}^{-1} f_{\neq}\right\|_{H^{\sigma}},
\end{aligned}
$$

and similarly we have

$\left\|E_{L H}^{2}\right\|_{H^{\sigma}}$

$$
\begin{aligned}
& \lesssim\left\|\langle k, \eta\rangle^{\sigma} \sqrt{\frac{\partial_{t} w_{k}(t, \eta)}{w_{k}(t, \eta)}} 1_{t \in I_{k, \eta}} 1_{k \neq 0} \int_{|\xi| \geq|\eta-\xi|} \widehat{v^{\prime \prime}}(\eta-\xi)|\xi-k t| \widehat{\phi}_{k}(t, \xi) d \xi\right\|_{L^{2}} \\
& \lesssim\left\|1_{t \in I_{k, \eta}} 1_{k \neq 0} \int_{|\xi| \geq|\eta-\xi|}\langle\eta-\xi\rangle \widehat{v^{\prime \prime}}(\eta-\xi)|\xi-k t|\langle k, \xi\rangle^{\sigma} \sqrt{\frac{\partial_{t} w_{k}(t, \xi)}{w_{k}(t, \xi)}} \widehat{\phi}_{k}(t, \xi) d \xi\right\|_{L^{2}} \\
& \lesssim\left\|v^{\prime \prime}\right\|_{H^{6}}\left\|\sqrt{\frac{\partial_{t} w}{w}} \chi_{R} \Delta_{L} \Delta_{t}^{-1} f_{\neq}\right\|_{H^{\sigma}} \lesssim \varepsilon v^{\frac{1}{3}}\left\|\sqrt{\frac{\partial_{t} w}{w}} \chi_{R} \Delta_{L} \Delta_{t}^{-1} f_{\neq}\right\|_{H^{\sigma}} .
\end{aligned}
$$

Finally, we deal with $T_{H L}^{2}$ : we have $\sqrt{\frac{\partial_{t} w_{k}(t, \xi)}{w_{k}(t, \xi)}} \lesssim \frac{k t}{\eta}$ and then we get

$$
\begin{aligned}
\left\|E_{H L}^{2}\right\|_{H^{\sigma}} & \lesssim\left\|\langle k, \eta\rangle^{\sigma} \frac{k t}{\eta} 1_{t \in I_{k, \eta}} 1_{k \neq 0} \int_{|\xi| \geq|\eta-\xi|} \widehat{v^{\prime \prime}}(\xi)(\eta-\xi-k t) \widehat{\phi}_{k}(t, \eta-\xi) d \xi\right\|_{L^{2}} \\
& \lesssim\left\|1_{t \in I_{k, \eta}} 1_{k \neq 0} \int_{|\xi| \geq|\eta-\xi|}\langle\xi\rangle^{\sigma-1}\left|\widehat{v^{\prime \prime}}(\xi)\right||k t||\eta-\xi-k t|\left|\widehat{\phi}_{k}(t, \eta-\xi)\right| d \xi\right\|_{L^{2}}
\end{aligned}
$$




$$
\begin{aligned}
& \lesssim t\left\|v^{\prime \prime}\right\|_{H^{\sigma-1}}\left\|\nabla_{L} \phi_{\neq}\right\|_{H^{4}} \\
& \lesssim\|h\|_{H^{\sigma}}\left\|f_{\neq}\right\|_{H^{5}} \lesssim \frac{\varepsilon^{2} v^{\frac{1}{2}}}{\left\langle v t^{3}\right\rangle} .
\end{aligned}
$$

Thus we have proved the lemma.

By the fact that $u=(0, g)^{T}+(1+h) \nabla_{z, v}^{\perp} P_{\neq} \phi$, Lemma 4.1 and under the bootstrap hypotheses, it holds that

$$
\|u\|_{H^{s}} \lesssim\|g\|_{H^{s}}+\left\|P_{\neq} \phi\right\|_{H^{s+1}} \lesssim \frac{\varepsilon v^{\frac{1}{3}}}{\left\langle t^{2}\right\rangle} .
$$

Lemma 4.4. Under the bootstrap hypotheses for $\varepsilon$ sufficiently small, for $s \leq \sigma-7$ it holds that

$$
\left\|A_{E}^{s}\left(P_{\neq} \phi\right)\right\|_{2} \lesssim \frac{1}{\langle t\rangle^{2}}\left(\left\|A_{E}^{s} f\right\|_{2}+\|f\|_{H^{\sigma}}\right) .
$$

Proof. By Lemma 3.4, we have

$$
\begin{aligned}
\left\|A_{E}^{s}\left(\nabla^{\perp} P_{\neq} \phi\right)\right\|_{2}^{2} \approx & \left\|v \max \left\{t^{3}, \eta^{3}\right\} \widehat{\phi}_{\neq}\right\|_{H^{s}}^{2} \\
\lesssim & v^{2} \sum_{k \neq 0} \int_{2|\eta| \geq t}\langle k, \eta\rangle^{2 s+6}\left|\widehat{\phi}_{k}(t, \eta)\right|^{2} d \eta \\
& +v^{2} \sum_{k \neq 0} \int_{2|\eta|<t} t^{6}\langle k, \eta\rangle^{2 s}\left|\widehat{\phi}_{k}(t, \eta)\right|^{2} d \eta \\
& =\Pi_{1}+\Pi_{2} .
\end{aligned}
$$

By Lemma 4.1 we get

$$
\left|\Pi_{1}\right| \lesssim\left\|P_{\neq} \phi\right\|_{H^{s+3}}^{2} \lesssim \frac{1}{\langle t\rangle^{4}}\left\|f_{\neq}\right\|_{H^{s+5}}^{2} \lesssim \frac{1}{\langle t\rangle^{4}}\left\|f_{\neq}\right\|_{H^{\sigma}}^{2}
$$

and

$$
\begin{aligned}
\left|\Pi_{2}\right| \lesssim v^{2} t^{6}\left\|P_{\neq} \phi\right\|_{H^{s}}^{2} & \lesssim v^{2} \sum_{k \neq 0} \int_{2|\eta|<t} t^{6} \frac{\langle k, \eta\rangle^{2 s}}{\left(k^{2}+|\eta-k t|^{2}\right)^{2}}\left|\widehat{\Delta_{L}} \phi_{k}(t, \eta)\right|^{2} d \eta \\
& \lesssim v^{2} \sum_{k \neq 0} \int_{2|\eta|<t} t^{6} \frac{\langle k, \eta\rangle^{2 s}}{\left(k^{2}+|\eta|^{2}+k^{2} t^{2}\right)^{2}}\left|\widehat{\Delta_{L}} \phi_{k}(t, \eta)\right|^{2} d \eta \\
& \lesssim \frac{1}{\langle t\rangle^{4}}\left\|v t^{3} \Delta_{L} \phi_{\neq}\right\|_{H^{s}}^{2} .
\end{aligned}
$$

By Lemma 4.1, we then obtain

$$
\left|\Pi_{2}\right| \lesssim \frac{1}{\langle t\rangle^{4}}\left\|v t^{3} f_{\neq}\right\|_{H^{s}}^{2} \lesssim \frac{1}{\langle t\rangle^{4}}\left\|A_{E}^{s} f_{\neq}\right\|_{2}^{2} .
$$

Thus we have proved the lemma. 


\section{Dissipation error term}

In this section we will deal with the dissipation error term in (2.45).

\subsection{Treatment of the zero mode}

By the fact that

$$
A_{0}^{\sigma}(\eta) \approx\langle\eta\rangle^{\sigma} \approx 1+|\eta|^{\sigma} \quad \text { and } \quad|\eta| \leq|\xi|+|\eta-\xi| \lesssim \max \{|\xi|,|\eta-\xi|\},
$$

we get

$$
\begin{aligned}
& \left.\left|E^{0}\right| \lesssim \int_{\xi, \eta}\langle\eta\rangle^{2 \sigma}\left|\overline{\hat{f}}_{0}(\eta)\right| \mid \widehat{\left(1-\left(v^{\prime}\right)^{2}\right.}(\eta-\xi)\right)|\xi|^{2} \hat{f}_{0}(\xi) \mid d \xi d \eta \\
& \lesssim \int_{\xi, \eta} 1_{|\eta| \leq 1}\left|\overline{\hat{f}}_{0}(\eta)\right|\left|\left(\overline{1-\left(v^{\prime}\right)^{2}}(\eta-\xi)\right)\right| \xi\left|(|\eta|+|\xi-\eta|) \hat{f}_{0}(\xi)\right| d \eta d \xi \\
& \left.+\int_{\xi, \eta} 1_{|\eta| \geq 1} 1_{|\xi-\eta| \geq|\xi|}|\eta|^{2 \sigma}\left|\overline{\hat{f}}_{0}(\eta)\right| \mid \widehat{\left(1-\left(v^{\prime}\right)^{2}\right.}(\eta-\xi)\right)|\xi|^{2} \hat{f}_{0}(\xi) \mid d \xi d \eta \\
& +\left.\int_{\xi, \eta} 1_{|\eta| \geq 1} 1_{|\xi-\eta|<|\xi|}|\eta|^{2 \sigma}\left|\overline{\hat{f}}_{0}(\eta)\right|\left|\left(\widehat{1-\left(v^{\prime}\right)^{2}}(\eta-\xi)\right)\right| \xi\right|^{2} \hat{f}_{0}(\xi) \mid d \xi d \eta \\
& \left.\lesssim \int_{\xi, \eta} 1_{|\eta| \leq 1}\left|{\widehat{\partial_{v} f}}_{0}(\eta)\right| \mid \widehat{\left(1-\left(v^{\prime}\right)^{2}\right.}(\eta-\xi)\right)|\xi| \hat{f}_{0}(\xi) \mid d \eta d \xi \\
& \left.+\int_{\xi, \eta} 1_{|\eta| \leq 1}\left|\overline{\hat{f}}_{0}(\eta)\right| \mid \overline{\left(\partial_{v}\left(1-\left(v^{\prime}\right)^{2}\right)\right.}(\eta-\xi)\right)|\xi| \hat{f}_{0}(\xi) \mid d \eta d \xi \\
& \left.+\int_{\xi, \eta} 1_{|\eta| \geq 1} 1_{|\xi-\eta|<|\xi|}|\eta|^{\sigma+1}\left|\overline{\hat{f}}_{0}(\eta)\right| \mid \widehat{\left(1-\left(v^{\prime}\right)^{2}\right.}(\eta-\xi)\right)|\xi|^{\sigma+1} \hat{f}_{0}(\xi) \mid d \xi d \eta \\
& +\int_{\xi, \eta} 1_{|\eta| \geq 1} 1_{|\xi-\eta| \geq|\xi|}|\eta|^{\sigma+1}\left|\overline{\hat{f}}_{0}(\eta)\right|\left|\left(\widehat{1-\left(v^{\prime}\right)^{2}}(\eta-\xi)\right)\right| \eta-\left.\xi\right|^{\sigma-1}|\xi|^{2} \hat{f}_{0}(\xi) \mid d \xi d \eta \\
& \lesssim v\left\|\partial_{v} f_{0}\right\|_{H^{2}}^{2}\left\|1-\left(v^{\prime}\right)^{2}\right\|_{2}+v\left\|\partial_{v} f_{0}\right\|_{H^{2}}\left\|\partial_{v}\left(1-\left(v^{\prime}\right)^{2}\right)\right\|_{2}\left\|f_{0}\right\|_{2} \\
& +v\left\|\partial_{v} f_{0}\right\|_{H^{\sigma}}^{2}\left\|1-\left(v^{\prime}\right)^{2}\right\|_{H^{2}}+v\left\|\partial_{v} f_{0}\right\|_{H^{\sigma}}\left\|\partial_{v}\left(1-\left(v^{\prime}\right)^{2}\right)\right\|_{H^{\sigma-2}}\left\|f_{0}\right\|_{H^{4}} \text {. }
\end{aligned}
$$

The purpose of the above estimate is to obtain the homogeneous derivative. By the fact that $\left(v^{\prime}\right)^{2}-1=\left(1-\left(v^{\prime}\right)\right)^{2}+2\left(v^{\prime}-1\right)=h^{2}+2 h$ and

$$
\left\|h^{2}\right\|_{H^{s}} \lesssim\|h\|_{H^{1}}\|h\|_{H^{s}}, \quad\left\|\partial_{v} h^{2}\right\|_{H^{s}} \lesssim\|h\|_{H^{1}}\left\|\partial_{v} h\right\|_{H^{s}}, \quad s \geq 1,
$$

we obtain by the bootstrap hypotheses that

$$
\begin{aligned}
\left|E^{0}\right| & \lesssim v\left(\|h\|_{H^{2}}+1\right)\left(\left\|\partial_{v} A^{\sigma} f_{0}\right\|_{2}^{2}\|h\|_{H^{2}}+\left\|\partial_{v} h\right\|_{H^{\sigma-2}}\left\|\partial_{v} f_{0}\right\|_{H^{\sigma}}\left\|f_{0}\right\|_{H^{4}}\right) \\
& \lesssim \varepsilon v^{\frac{1}{3}} v\left\|\partial_{v} A^{\sigma} f_{0}\right\|_{2}^{2}+\varepsilon v^{\frac{1}{3}} v\left\|\partial_{v} h\right\|_{H^{\sigma-1}}^{2} .
\end{aligned}
$$

\subsection{Treatment of the nonzero mode}

We use a paraproduct decomposition in $v$. Then we have

$$
E^{\neq}=E_{L H}^{\neq}+E_{H L}^{\neq}+E_{H H}^{\neq},
$$


where

$$
\begin{aligned}
& E_{L H}^{\neq}=-\sum_{M \geq 8} v \int A^{\sigma} f_{\neq} A^{\sigma}\left(\left(1-\left(v^{\prime}\right)^{2}\right)_{<M / 8}\left(\partial_{v}-t \partial_{z}\right)^{2}\left(f_{\neq}\right)_{M}\right) d z d v, \\
& E_{H L}^{\neq}=-\sum_{M \geq 8} v \int A^{\sigma} f_{\neq} A^{\sigma}\left(\left(1-\left(v^{\prime}\right)^{2}\right)_{M}\left(\partial_{v}-t \partial_{z}\right)^{2}\left(f_{\neq}\right)_{<M / 8}\right) d z d v, \\
& E_{H H}^{\neq}=-v \sum_{M \in \mathbb{D}} \sum_{\frac{1}{8} M \leq M^{\prime} \leq 8 M} \int A^{\sigma} f_{\neq} A^{\sigma}\left(\left(1-\left(v^{\prime}\right)^{2}\right)_{M}\left(\partial_{v}-t \partial_{z}\right)^{2}\left(f_{\neq}\right)_{M^{\prime}}\right) d z d v .
\end{aligned}
$$

\subsubsection{Treatment of $\boldsymbol{E}_{\boldsymbol{L} \boldsymbol{H}}^{\not \neq}$. We have}

$$
\left.E_{L H}^{\neq} \lesssim v \sum_{M \geq 8} \sum_{k \neq 0} \int_{\eta, \xi} A^{\sigma}\left|\overline{\hat{f}}_{k}(\eta)\right| A_{k}^{\sigma}(\eta) \mid \widehat{\left(1-\left(v^{\prime}\right)^{2}\right.}\right)(\eta-\xi)_{<M / 8}|\xi-k t|^{2} \hat{f}_{k}(\xi)_{M} \mid d \xi d \eta .
$$

By the fact that $\xi \approx \eta \approx M,|k, \eta| \approx|k, \xi|$ and

$$
|\xi-k t| \lesssim|\xi-\eta|+|\eta-k t| \lesssim\langle\xi-\eta\rangle \sqrt{k^{2}+|\eta-k t|^{2}}
$$

we have

$$
\begin{aligned}
E_{L H}^{\neq} \lesssim v \sum_{M \geq 8} \sum_{k \neq 0} \int_{\eta, \xi} \sqrt{k^{2}+|\eta-k t|^{2}} A^{\sigma}\left|\overline{\hat{f}}_{k}(\eta)\right| \mid \overline{\left\langle\partial_{v}\right\rangle\left(1-\left(v^{\prime}\right)^{2}\right)}(\eta-\xi)_{<M / 8} \\
\times|\xi-k t| A_{k}^{\sigma}(\xi) \hat{f}_{k}(\xi)_{M} \mid d \xi d \eta \\
\therefore v \sum_{M \geq 8}\left\|\left(\sqrt{-\Delta_{L}} A^{\sigma} f_{\neq}\right)_{\sim M}\right\|_{2}\left\|\left(\sqrt{-\Delta_{L}} A^{\sigma} f_{\neq}\right)_{M}\right\|_{2}\left\|\left(1-\left(v^{\prime}\right)^{2}\right)\right\|_{H^{4}},
\end{aligned}
$$

which gives

$$
E_{L H}^{\not=} \lesssim v\left\|\left(\sqrt{-\Delta_{L}} A^{\sigma} f_{\neq}\right)\right\|_{2}^{2}\left\|\left(1-\left(v^{\prime}\right)^{2}\right)\right\|_{H^{4}} .
$$

5.2.2. Treatment of $\boldsymbol{E}_{\boldsymbol{H} L}^{\neq}$. We have

$$
\begin{aligned}
E_{H L}^{\neq} \lesssim v \sum_{M \geq 8} \sum_{k \neq 0} \int_{\eta, \xi}\left[1_{|\eta| \leq 16|k|}+1_{|\eta|>16|k|}\right] A^{\sigma}\left|\overline{\hat{f}}_{k}(\eta)\right| A_{k}^{\sigma}(\eta) \\
\\
\quad \times\left|\widehat{\left(1-\left(v^{\prime}\right)^{2}\right)}(\eta-\xi)_{M}\right| \xi-\left.k t\right|^{2} \hat{f}_{k}(\xi)_{<M / 8} \mid d \xi d \eta \\
\quad=E_{H L}^{\neq, z}+E_{H L}^{\neq, v} .
\end{aligned}
$$

For $E_{H L}^{\neq, z}$, we have $|k, \eta| \approx|k| \approx|k, \xi|$ and

$$
|\xi-k t| \lesssim|\xi-\eta|+|\eta-k t| \lesssim\langle\xi-\eta\rangle \sqrt{k^{2}+|\eta-k t|^{2}},
$$


which then implies

$$
\begin{aligned}
E_{H L}^{\neq, z} \lesssim v \sum_{M \geq 8} \sum_{k \neq 0} \int_{\eta, \xi} 1_{|\eta| \leq 16|k|} A^{\sigma} \sqrt{k^{2}+|\eta-k t|^{2}}\left|\overline{\hat{f}}_{k}(\eta)\right| \\
\left.\quad \times \mid\langle\eta-\xi\rangle \widehat{\left(1-\left(v^{\prime}\right)^{2}\right.}\right)(\eta-\xi)_{M}|\xi-k t||k|^{\sigma} \hat{f}_{k}(\xi)_{<M / 8} \mid d \xi d \eta \\
\lesssim v \sum_{M \geq 8} M^{-2}\left\|\left(1-\left(v^{\prime}\right)^{2}\right)_{M}\right\|_{H^{5}}\left\|\left(\sqrt{-\Delta_{L}} A^{\sigma} f_{\neq}\right)\right\|_{2}^{2} .
\end{aligned}
$$

Thus we have

$$
E_{H L}^{\neq, z} \lesssim v\left\|\left(1-\left(v^{\prime}\right)^{2}\right)\right\|_{H^{5}}\left\|\left(\sqrt{-\Delta_{L}} A^{\sigma} f_{\neq}\right)\right\|_{2}^{2} .
$$

We turn to $E_{H L}^{\neq, v}$. In this case, $|k, \eta| \approx|\eta| \approx|\eta-\xi| \approx M$, and then we get

$$
\begin{aligned}
&\left.\left|E_{H L}^{\neq, v}\right| \lesssim v \sum_{M \geq 8} \sum_{k \neq 0} \int_{\eta, \xi} 1_{|\eta|>16|k|} A^{\sigma}\left|\overline{\hat{f}}_{k}(\eta)\right||\eta-\xi|\langle\eta-\xi\rangle^{\sigma-1} \mid \widehat{\left(1-\left(v^{\prime}\right)^{2}\right.}\right)(\eta-\xi)_{M} \mid \\
& \quad \times|\xi-k t|^{2}\left|\hat{f}_{k}(\xi)_{<M / 8}\right| d \xi d \eta \\
& \lesssim v \sum_{M \geq 8}\left\|\left(f_{\neq}\right)_{\sim M}\right\|_{H^{\sigma}}\left\|\partial_{v}\left(1-\left(v^{\prime}\right)^{2}\right)_{M}\right\|_{H^{\sigma-1}}\langle t\rangle^{2}\left\|f_{\neq}\right\|_{H^{5}} \\
& \lesssim v\left\|f_{\neq}\right\|_{H^{\sigma}}\left\|\partial_{v}\left(1-\left(v^{\prime}\right)^{2}\right)\right\|_{H^{\sigma-1}}\langle t\rangle^{2}\left\|f_{\neq}\right\|_{H^{5}}
\end{aligned}
$$

5.2.3. Treatment of $\boldsymbol{E}_{\boldsymbol{H} \boldsymbol{H}}^{\not \neq}$. In this case, it holds that $|\eta-\xi| \approx|\xi| \approx M^{\prime}$. We divide the problem into two parts:

$$
\begin{aligned}
\left|E_{H H}^{f}\right| \lesssim v \sum_{M \in \mathbb{D}} \sum_{\frac{1}{8} M \leq M^{\prime} \leq 8 M} \sum_{k \neq 0} \int_{\eta, \xi}\left[1_{|k| \geq 16|\xi|}+1_{|k|<16|\xi|}\right] \\
\left.\quad \times A^{\sigma}\left|\overline{\hat{f}}_{k}(\eta)\right| A_{k}^{\sigma}(\eta) \mid \widehat{\left(1-\left(v^{\prime}\right)^{2}\right.}\right)(\eta-\xi)_{M^{\prime}}|\xi-k t|^{2} \hat{f}_{k}(\xi)_{M} \mid d \xi d \eta \\
=E_{H H}^{\neq, z}+E_{H H}^{\neq, v} .
\end{aligned}
$$

To treat $E_{H H}^{\neq, z}$, we have

$$
|k| \lesssim|k, \eta| \lesssim|k|+|\eta-\xi|+|\xi| \lesssim|k|
$$

and

$$
|\xi-k t| \lesssim|\xi-\eta|+|\eta-k t| \lesssim\langle\xi-\eta\rangle \sqrt{k^{2}+|\eta-k t|^{2}} .
$$

Therefore we get

$$
\begin{aligned}
& E_{H H}^{\neq, z} \lesssim v \sum_{M \in \mathbb{D}} \sum_{\frac{1}{8}} \sum_{M \leq M^{\prime} \leq 8 M} \int_{\eta \neq 0} 1_{|k| \geq 16|\xi|} A^{\sigma} \sqrt{k^{2}+|\eta-k t|^{2}}\left|\overline{\hat{f}}_{k}(\eta)\right| \\
&\left.\quad \times\langle\xi-\eta\rangle \mid \widehat{\left(1-\left(v^{\prime}\right)^{2}\right.}\right)(\eta-\xi)_{M^{\prime}}|\xi-k t||k|^{\sigma} \hat{f}_{k}(\xi)_{M} \mid d \xi d \eta \\
& \lesssim v \sum_{M \in \mathbb{D}}\left\|\sqrt{-\Delta_{L}} A^{\sigma} f\right\|_{2}\left\|\left(\sqrt{-\Delta_{L}} A^{\sigma} f\right)_{M}\right\|_{2}\left\|\left(1-\left(v^{\prime}\right)^{2}\right)_{\sim}\right\|_{H^{3}} \\
& \lesssim v\left\|\sqrt{-\Delta_{L}} A^{\sigma} f\right\|_{2}^{2}\left\|\left(1-\left(v^{\prime}\right)^{2}\right)\right\|_{H^{3}} .
\end{aligned}
$$


Next we turn to $E_{H H}^{\neq, v}$, in which case we have

$$
|k, \eta| \lesssim|k|+|\eta| \lesssim|k|+|\eta-\xi|+|\xi| \approx|k|+|\xi| \lesssim|\xi| \approx|\xi-\eta|
$$

and

$$
|\xi-k t| \lesssim|\xi-\eta|+|\eta-k t| \lesssim\langle\xi-\eta\rangle \sqrt{k^{2}+|\eta-k t|^{2}} .
$$

Therefore we get

$$
\begin{aligned}
E_{H H}^{\neq, v} \lesssim v \sum_{M \in \mathbb{D}} \sum_{\frac{1}{8}} \sum_{M \leq M^{\prime} \leq 8 M} \int_{k \neq 0} \int_{\eta, \xi} 1_{|k|<16|\xi|} A^{\sigma} \sqrt{k^{2}+|\eta-k t|^{2}}\left|\overline{\hat{f}}_{k}(\eta)\right| \\
\left.\quad \times\langle\xi-\eta\rangle \mid \widehat{\left(1-\left(v^{\prime}\right)^{2}\right.}\right)(\eta-\xi)_{M^{\prime}}|\xi-k t||\xi|^{\sigma} \hat{f}_{k}(\xi)_{M} \mid d \xi d \eta \\
\lesssim v \sum_{M \in \mathbb{D}}\left\|\sqrt{-\Delta_{L}} A^{\sigma} f\right\|_{2}\left\|\left(\sqrt{-\Delta_{L}} A^{\sigma} f\right)_{M}\right\|_{2}\left\|\left(1-\left(v^{\prime}\right)^{2}\right)_{\sim}\right\|_{H^{3}} \\
\lesssim v\left\|\sqrt{-\Delta_{L}} A^{\sigma} f\right\|_{2}^{2}\left\|\left(1-\left(v^{\prime}\right)^{2}\right)\right\|_{H^{3}} .
\end{aligned}
$$

By the fact that $\left(v^{\prime}\right)^{2}-1=h^{2}+2 h$, the bootstrap hypotheses and (2.28), we obtain

$$
\begin{aligned}
\left|E^{\neq}\right| \lesssim & v\left\|f_{\neq}\right\|_{H^{\sigma}}\left\|\partial_{v}\left(1-\left(v^{\prime}\right)^{2}\right)\right\|_{H^{\sigma-1}}\langle t\rangle^{2}\left\|f_{\neq}\right\|_{H^{5}} \\
& +v\left\|\sqrt{-\Delta_{L}} A^{\sigma} f\right\|_{2}^{2}\left\|\left(1-\left(v^{\prime}\right)^{2}\right)\right\|_{H^{4}} \\
\lesssim & v\left(1+\|h\|_{H^{2}}\right)\left(\left\|f_{\neq}\right\|_{H^{\sigma}}\left\|\partial_{v} h\right\|_{H^{\sigma-1}}\langle t\rangle^{2}\left\|f_{\neq}\right\|_{H^{5}}+\left\|\sqrt{-\Delta_{L}} A^{\sigma} f\right\|_{2}^{2}\|h\|_{H^{4}}\right) \\
\lesssim & v \varepsilon v^{\frac{1}{3}}\left\|\sqrt{-\Delta_{L}} A^{\sigma} f\right\|_{2}^{2}+\left(\varepsilon v^{\frac{1}{3}}\right)^{2} v\left\|\partial_{v} h\right\|_{H^{\sigma-1}} \frac{\langle t\rangle^{2}}{\left\langle v t^{3}\right\rangle}
\end{aligned}
$$

We end the section by proving Proposition 2.5.

Proof of Proposition 2.5. We get by (2.45) that

$$
\begin{aligned}
& \int_{1}^{t}\left(v \int A^{\sigma} f\left(t^{\prime}\right) A^{\sigma}\left(\widetilde{\Delta}_{t} f\left(t^{\prime}\right)\right) d z d v\right) d t^{\prime} \\
& \quad \leq-\int_{1}^{t} v\left\|\sqrt{-\Delta_{L}} A^{\sigma} f\left(t^{\prime}\right)\right\|_{2}^{2} d t^{\prime}+\int_{1}^{t}\left|E^{\neq}\left(t^{\prime}\right)\right|+\left|E^{0}\left(t^{\prime}\right)\right| d t^{\prime} .
\end{aligned}
$$

Then by (5.1) and (5.2) we obtain

$$
\begin{aligned}
\int_{1}^{t}(v & \left.\int A^{\sigma} f\left(t^{\prime}\right) A^{\sigma}\left(\tilde{\Delta}_{t} f\left(t^{\prime}\right)\right) d z d v\right) d t^{\prime} \\
\leq & -\int_{1}^{t} v\left\|\sqrt{-\Delta_{L}} A^{\sigma} f\left(t^{\prime}\right)\right\|_{2}^{2} d t^{\prime}+C \varepsilon v^{\frac{1}{3}} \int_{1}^{t} v\left\|\sqrt{-\Delta_{L}} A^{\sigma} f\left(t^{\prime}\right)\right\|_{2}^{2} d t^{\prime} \\
& +C \varepsilon v^{\frac{1}{3}} v\left\|\partial_{v} h\right\|_{L_{T}^{2}\left(H^{\sigma-1}\right)}^{2}+C \int_{1}^{t}\left(\varepsilon v^{\frac{1}{3}}\right)^{2} v\left\|\partial_{v} h\left(t^{\prime}\right)\right\|_{H^{\sigma-1}} \frac{\langle t\rangle^{2}}{\left\langle v t^{\prime 3}\right\rangle} d t^{\prime}
\end{aligned}
$$


Thus, by taking $\varepsilon$ small enough and using Proposition 2.2 we get

$$
\begin{aligned}
& \int_{1}^{t}\left(v \int A^{\sigma} f\left(t^{\prime}\right) A^{\sigma}\left(\widetilde{\Delta}_{t} f\left(t^{\prime}\right)\right) d z d v\right) d t^{\prime} \\
& \quad \leq-\frac{7}{8} \int_{1}^{t} v\left\|\sqrt{-\Delta_{L}} A^{\sigma} f\left(t^{\prime}\right)\right\|_{2}^{2} d t^{\prime}+\varepsilon^{2} v\left\|\partial_{v} h\left(t^{\prime}\right)\right\|_{L_{T}^{2} H^{\sigma-1}}\left(\int_{1}^{t} \frac{1}{\left\langle v^{\frac{1}{3}} t^{\prime}\right\rangle^{2}} d t^{\prime}\right)^{\frac{1}{2}} \\
& \quad \leq-\frac{7}{8} \int_{1}^{t} v\left\|\sqrt{-\Delta_{L}} A^{\sigma} f\left(t^{\prime}\right)\right\|_{2}^{2} d t^{\prime}+C \varepsilon^{3} v^{\frac{2}{3}} .
\end{aligned}
$$

Thus we have proved Proposition 2.5.

\section{Transport}

To treat the transport term, we need to consider the commutator. The following lemma gives the key commutator estimate.

Lemma 6.1. Assume that $|\xi-\eta| \leq \frac{1}{10}|\eta|$; then it holds that

$$
|w(t, \eta)-w(t, \xi)| \lesssim \frac{|\xi-\eta|}{\langle\eta\rangle} \times \begin{cases}v^{-\frac{1}{3}}, & t \lesssim v^{-\frac{1}{3}} \\ v^{-\frac{1}{3} \beta} t^{1-\beta}, & t \gtrsim v^{-\frac{1}{3}}\end{cases}
$$

Let us admit the lemma and finish the estimate of transport term first. Then the proof of the lemma will be presented at the end of this section.

We write

$$
\begin{aligned}
T_{N}= & i \sum_{k, l} \int_{\eta, \xi} A_{k}^{\sigma}(\eta) \overline{\hat{f}}_{k}(\eta) \hat{u}_{k-l}(\eta-\xi)_{<N / 8} \cdot(l, \xi) A_{l}^{\sigma}(\xi) \hat{f}_{l}(\xi)_{N}\left(\frac{A_{k}^{\sigma}(\eta)}{A_{l}^{\sigma}(\xi)}-1\right) d \xi d \eta \\
= & i \sum_{k, l} \int_{\eta, \xi} A_{k}^{\sigma}(\eta) \overline{\hat{f}}_{k}(\eta) \hat{u}_{k-l}(\eta-\xi)_{<N / 8} \cdot(l, \xi) A_{l}^{\sigma}(\xi) \hat{f}_{l}(\xi)_{N} \\
& \times\left(\frac{\langle k, \eta\rangle^{\sigma}}{\langle l, \xi\rangle^{\sigma}}-1\right) \frac{w_{l}(t, \xi)}{w_{k}(t, \eta)} d \xi d \eta \\
& +i \sum_{k, l} \int_{\eta, \xi} A_{k}^{\sigma}(\eta) \overline{\hat{f}}_{k}(\eta) \hat{u}_{k-l}(\eta-\xi)_{<N / 8} \cdot(l, \xi) A_{l}^{\sigma}(\xi) \hat{f}_{l}(\xi)_{N} \\
& \times\left(\frac{w_{l}(t, \xi)}{w_{k}(t, \eta)}-1\right) d \xi d \eta \\
= & T_{N}^{1}+T_{N}^{2} .
\end{aligned}
$$

For the first term we get

$$
\left|\frac{\langle k, \eta\rangle^{\sigma}}{\langle l, \xi\rangle^{\sigma}}-1\right| \lesssim \frac{\langle k-l, \eta-\xi\rangle}{\langle l, \xi\rangle}
$$


which gives

$$
\left|T_{N}^{1}\right| \lesssim\left\|A^{\sigma} f_{\sim N}\right\|_{2}\left\|A^{\sigma} f_{N}\right\|_{2}\|u\|_{H^{4}} .
$$

Next we will deal with $T_{N}^{2}$. By the support of the integrand we get

$$
\frac{N}{16} \leq|k-l, \xi-\eta| \leq \frac{3 N}{16}, \quad \frac{N}{2} \leq|l, \xi| \leq \frac{3 N}{2} .
$$

We then set more restrictions on the support of the integrand to make $k, \eta$ and $l, \xi$ closer. We get

$$
\begin{aligned}
T_{N}^{2}=i \sum_{k, l} \int_{\eta, \xi}\left(\chi^{D}+\left(1-\chi^{D}\right)\right) A_{k}(\eta) \overline{\hat{f}}_{k}(\eta) \hat{u}_{k-l}(\eta-\xi)_{<N / 8} \cdot(l, \xi) A_{l}(\xi) \hat{f}_{l}(\xi)_{N} \\
\quad \times\left(\frac{w_{l}(t, \xi)}{w_{k}(t, \eta)}-1\right) d \xi d \eta \\
=T_{N, D}^{2}+T_{N, *}^{2}
\end{aligned}
$$

where $\chi^{D}$ is a characteristic function (the indicator function) of the set

$$
D=\left\{(k, l, \xi, \eta):|k-l, \xi-\eta| \leq \frac{1}{1000}|l, \xi|\right\} .
$$

Then, by $\left|\frac{w(t, \xi)}{w(t, \eta)}\right| \lesssim 1$, we get

$$
\left|T_{N, *}^{2}\right| \lesssim\left\|A^{\sigma} f_{\sim N}\right\|_{2}\left\|A^{\sigma} f_{N}\right\|_{2}\|u\|_{H^{4}}
$$

We rewrite $T_{N, D}^{2}$ as

$$
\begin{aligned}
& T_{N, D}^{2}= i \sum_{k \neq l} \int_{\eta, \xi} \chi^{D} A_{k}(\eta) \overline{\hat{f}}_{k}(\eta) \hat{u}_{k-l}(\eta-\xi)_{<N / 8} \cdot(l, \xi) A_{l}(\xi) \hat{f}_{l}(\xi)_{N} \\
& \times\left(\frac{w_{l}(t, \xi)}{w_{k}(t, \eta)}-1\right) d \xi d \eta \\
&+i \sum_{l} \int_{\eta, \xi} \chi^{D} A_{k}(\eta) \overline{\hat{f}}_{l}(\eta) \hat{u}_{0}(\eta-\xi)_{<N / 8} \cdot(l, \xi) A_{l}(\xi) \hat{f_{l}}(\xi)_{N} \\
& \quad \times\left(\frac{w_{l}(t, \xi)}{w_{l}(t, \eta)}-1\right) d \xi d \eta \\
&=T_{N, \neq}^{2}+T_{N,=}^{2}
\end{aligned}
$$

\subsection{Treatment of $T_{N,=}^{2}$}

By the fact that $u_{0}=(0, g)$ and since for $|l| \geq 20 \max \{|\xi|,|\eta|\}$, we have $w_{l}(t, \xi)=$ $w_{l}(t, \eta)=w\left(t, \frac{l}{20}\right)$, we get

$$
\begin{aligned}
T_{N,=}^{2}=i \sum_{0 \neq l \leq 20 \max \{|\xi|,|\eta|\}} \int_{\eta, \xi} & \chi^{D} A_{k}(\eta) \overline{\hat{f}}(\eta) \hat{g}(\eta-\xi)_{<N / 8} \cdot \xi A_{l}(\xi) \hat{f}_{l}(\xi)_{N} \\
& \times\left(\frac{w_{l}(t, \xi)}{w_{l}(t, \eta)}-1\right) d \xi d \eta .
\end{aligned}
$$


Due to the fact that $0 \neq l \leq 20 \max \{|\xi|,|\eta|\} \approx|\xi|$, we get $\varrho(l, \eta) \approx|\eta|$. Thus, by Lemmas 6.1 and 3.1 we obtain

$$
\begin{aligned}
\left|\frac{w_{l}(t, \xi)}{w_{l}(t, \eta)}-1\right| & \lesssim|w(t, \varrho(l, \xi))-w(t, \varrho(l, \eta))| \\
& \lesssim\left(v^{-\frac{1}{3}} \chi_{t \lesssim v^{-\frac{1}{3}}}(t)+v^{-\frac{1}{3} \beta} t^{1-\beta} \chi_{t \gtrsim v^{-\frac{1}{3}}}(t)\right) \frac{|\varrho(l, \xi)-\varrho(l, \eta)|}{|\varrho(l, \eta)|} \\
& \lesssim\left(v^{-\frac{1}{3}} \chi_{t \lesssim v^{-\frac{1}{3}}}(t)+v^{-\frac{1}{3} \beta} t^{1-\beta} \chi_{t \gtrsim v^{-\frac{1}{3}}}(t)\right) \frac{|\eta-\xi|}{\eta} .
\end{aligned}
$$

Therefore we get

$$
\begin{aligned}
& \left|T_{N,=}^{2}\right| \lesssim\left\|A^{\sigma} f_{\sim N}\right\|_{2}\left\|A^{\sigma} f_{N}\right\|_{2}\|g\|_{H^{4}} \nu^{-\frac{1}{3}} \chi_{t \lesssim \nu^{-\frac{1}{3}}}(t)
\end{aligned}
$$

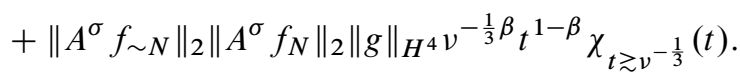

\subsection{Treatment of $T_{N, \neq}^{2}$}

By the definition of $\varrho(k, \eta)$, we have for $(l, k, \xi, \eta) \in D$ that

$$
|\varrho(k, \eta)| \approx|\varrho(l, \xi)| \approx|l, \xi| \text {. }
$$

We get by Lemmas 6.1 and 3.1 that

$$
\begin{aligned}
\left|\frac{w_{l}(t, \xi)}{w_{k}(t, \eta)}-1\right| & \lesssim|w(t, \varrho(l, \xi))-w(t, \varrho(k, \eta))| \\
& \lesssim \frac{|\varrho(l, \xi)-\varrho(k, \eta)|}{|\varrho(k, \eta)|}\left(v^{-\frac{1}{3}} \chi_{t \lesssim v^{-\frac{1}{3}}}(t)+v^{-\frac{1}{3} \beta} t^{1-\beta} \chi_{t \gtrsim v^{-\frac{1}{3}}}(t)\right) \\
& \lesssim \frac{|l-k, \xi-\eta|}{|l, \xi|}\left(v^{-\frac{1}{3}} \chi_{t \lesssim v^{-\frac{1}{3}}}(t)+v^{-\frac{1}{3} \beta} t^{1-\beta} \chi_{t \gtrsim v^{-\frac{1}{3}}}(t)\right),
\end{aligned}
$$

which implies that

$$
\left|T_{N, \neq}^{2}\right| \lesssim\left\|A^{\sigma} f_{\sim N}\right\|_{2}\left\|A^{\sigma} f_{N}\right\|_{2}\left\|u_{\neq}\right\|_{H^{4}}\left(v^{-\frac{1}{3}} \chi_{t \lesssim v^{-\frac{1}{3}}}(t)+v^{-\frac{1}{3} \beta} t^{1-\beta} \chi_{t \gtrsim v^{-\frac{1}{3}}}(t)\right) .
$$

By the fact that

$$
u_{\neq}=h \nabla_{z, v}^{\perp} P_{\neq} \phi+\nabla_{z, v}^{\perp} P_{\neq} \phi,
$$

we then get by Lemma 4.1 (by taking $s^{\prime}=2$ in the lemma) that

$$
\left\|u_{\neq}\right\|_{H^{4}} \lesssim\left(1+\|h\|_{H^{4}}\right)\left\|\nabla_{z, v}^{\perp} P_{\neq} \phi\right\|_{H^{4}} \lesssim \frac{1}{\langle t\rangle^{2}}\left(1+\|h\|_{H^{4}}\right)\left\|f_{\neq}\right\|_{H^{7}} .
$$

Therefore we get

$$
\begin{aligned}
\left|T_{N}\right| \lesssim & \left\|A^{\sigma} f_{\sim N}\right\|_{2}\left\|A^{\sigma} f_{N}\right\|_{2}\left(\|g\|_{H^{4}}+\left\|u_{\neq}\right\|_{H^{4}}\right) \\
& \times\left(v^{-\frac{1}{3}} \chi_{t \lesssim \nu^{-\frac{1}{3}}}(t)+v^{-\frac{1}{3} \beta} t^{1-\beta} \chi_{t \gtrsim v^{-\frac{1}{3}}}(t)\right) \\
\lesssim & \varepsilon\left\|A^{\sigma} f_{\sim N}\right\|_{2}\left\|A^{\sigma} f_{N}\right\|_{2}\left(\frac{\chi_{t \lesssim \nu^{-\frac{1}{3}}}(t)}{\langle t\rangle^{2}}+\frac{v^{-\frac{1}{3} \beta+\frac{1}{3}} \chi_{t \gtrsim \nu^{-\frac{1}{3}}}(t)}{\langle t\rangle^{1+\beta}}\right) .
\end{aligned}
$$

Now we are able to prove Proposition 2.6. 
Proof of Proposition 2.6. We get by (6.1) and Proposition 2.2, (A.1) and (A.2) that

$$
\begin{aligned}
\int_{1}^{t} \sum_{N \geq 8}\left|T_{N}\left(t^{\prime}\right)\right| d t^{\prime} & \lesssim \varepsilon \int_{1}^{t} \sum_{N \geq 8}\left\|A^{\sigma} f_{\sim N}\right\|_{2}\left\|A^{\sigma} f_{N}\right\|_{2} \\
& \times\left(\frac{\chi_{t^{\prime} \lesssim v^{-\frac{1}{3}}}\left(t^{\prime}\right)}{\left\langle t^{\prime}\right\rangle^{2}}+\frac{v^{-\frac{1}{3} \beta+\frac{1}{3}} \chi_{t^{\prime} \gtrsim v^{-\frac{1}{3}}}\left(t^{\prime}\right)}{\left\langle t^{\prime}\right\rangle^{1+\beta}}\right) d t^{\prime} \\
& \lesssim \varepsilon \sup _{t^{\prime} \in[1, t]}\left\|A^{\sigma} f\left(t^{\prime}\right)\right\|_{2}^{2} .
\end{aligned}
$$

Thus we have proved Proposition 2.6.

\subsection{Proof of Lemma 6.1}

We end this section by proving Lemma 6.1.

Proof of Lemma 6.1. Without loss of the generality, we assume $0<\eta<\xi$. Then according to the relation between $t$ and $\xi, \eta$, we need to consider following five cases:

Case 1. For $t \leq t(\eta)$, we have $w(t, \eta)=w(t, \xi)=1$.

Case 2. For $t(\eta) \leq t \leq t(\xi)$, there exists $l \in[1, E(\sqrt{|\eta|})]$ such that $t \in I_{l, \eta}$; then $|l-E(\sqrt{|\eta|})| \lesssim \sqrt{\xi}-\sqrt{\eta}$ and

$$
\begin{aligned}
& |w(t, \eta)-w(t, \xi)|=|w(t, \eta)-1| \\
& \quad \leq\left|\prod_{m=E(\sqrt{|\eta|})}^{l+1} G_{m}(\eta) \exp \left(\left\langle v^{\frac{1}{3}} t_{l, \eta}\right\rangle^{-(1+\beta)} \frac{v^{\frac{1}{3}} \eta}{l^{2}}\left(\arctan \left(t-\frac{\eta}{l}\right)+\arctan \left(D_{l, \eta}^{-}\right)\right)\right)-1\right| \\
& \quad \leq \begin{cases}\exp \left(\sum_{m=E(\sqrt{|\eta|})}^{l} \frac{C v^{\frac{1}{3}} \eta}{m^{2}}\right)-1 \leq v^{\frac{1}{3}} \mid \sqrt{\xi}-\sqrt{\eta}, & \sqrt{\eta} \leq v^{-\frac{1}{3}}, \\
\exp \left(\sum_{m=E(\sqrt{|\eta|})}^{l} \frac{C l^{-1+\beta}}{\left(v^{\frac{1}{3}} \eta\right)^{\beta}}\right)-1 \leq \frac{|\sqrt{\xi}-\sqrt{\eta}|}{\sqrt{|\xi|}}\left(\frac{\sqrt{|\xi|}}{v^{\frac{1}{3}} \eta}\right)^{\beta}, \quad \sqrt{\eta}>v^{-\frac{1}{3}},\end{cases} \\
& \quad \lesssim|\eta-\xi|\langle\xi\rangle^{-1} .
\end{aligned}
$$

Here we use the fact that $\left|e^{x}-1\right| \lesssim|x|$ for $|x| \lesssim 1$.

Case 3. For $t(\xi) \leq t \leq 2 \eta$, there exist $k, l$ such that $t \in I_{k, \eta} \cap I_{l, \xi}$. By Lemma 3.2, we need to consider the following three cases.

(3a) $k=l$. Let

$$
\begin{aligned}
F_{1}(m, \eta) & =v^{\frac{1}{3}}\left\langle v^{\frac{1}{3}} t_{m, \eta}\right\rangle^{-(1+\beta)} \frac{\eta}{m^{2}} \\
F_{2}^{ \pm}(m, \eta) & =v^{\frac{1}{3}}\left\langle v^{\frac{1}{3}} t_{m, \eta}\right\rangle^{-(1+\beta)} \times \frac{\eta}{m^{2}} \arctan \left(D_{m, \eta}^{ \pm}\right)
\end{aligned}
$$


then $G_{m}(\eta)=e^{F^{+}(m, \eta)+F^{-}(m, \eta)}$ and we get

$$
\begin{gathered}
\left|\partial_{\eta} F_{1}(m, \eta)\right| \lesssim \frac{F_{1}(m, \eta)}{\langle\eta\rangle}, \quad\left|\partial_{\eta} F_{2}^{ \pm}(m, \eta)\right| \lesssim \frac{F_{2}^{ \pm}(m, \eta)}{\langle\eta\rangle}, \\
\left|\arctan \left(t-\frac{\eta}{l}\right)-\arctan \left(t-\frac{\xi}{l}\right)\right| \lesssim \min \left\{\frac{|\xi-\eta|}{l}, 1\right\}, \\
e^{x}-1 \leq\left(e^{x}+1\right)|x| \lesssim|x|, \quad \text { for }|x| \lesssim 1 .
\end{gathered}
$$

Therefore, we obtain

$$
\begin{aligned}
& |w(t, \eta)-w(t, \xi)|=w(t, \xi)\left|\frac{w(t, \eta)}{w(t, \xi)}-1\right| \\
& \lesssim \prod_{m=E(\sqrt{|\xi|})}^{m=E(\sqrt{|\eta|})+1} \frac{1}{G_{m}(\xi)} \prod_{m=E(\sqrt{|\eta|})}^{l+1} \frac{G_{m}(\eta)}{G_{m}(\xi)} \\
& \times \frac{\exp \left(v^{\frac{1}{3}}\left\langle v^{\frac{1}{3}} t_{l, \eta}\right\rangle^{-(1+\beta)} \frac{\eta}{l^{2}}\left(\arctan \left(t-\frac{\eta}{l}\right)+\arctan \left(D_{l, \eta}^{-}\right)\right)\right)}{\exp \left(v^{\frac{1}{3}}\left\langle v^{\frac{1}{3}} t_{l, \xi}\right\rangle^{-(1+\beta)} \frac{\xi}{l^{2}}\left(\arctan \left(t-\frac{\xi}{l}\right)+\arctan \left(D_{l, \xi}^{-}\right)\right)\right)}-1 \mid \\
& \lesssim\left|\prod_{m=E(\sqrt{|\xi|})}^{m=E(\sqrt{|\eta|})+1} \frac{1}{G_{m}(\xi)}-1\right|+\left|\prod_{m=E(\sqrt{|\eta|})}^{l+1} \frac{G_{m}(\eta)}{G_{m}(\xi)}-1\right| \\
& +\left|\frac{\exp \left(v^{\frac{1}{3}}\left\langle v^{\frac{1}{3}} t_{l, \eta}\right\rangle^{-(1+\beta)} \frac{\eta}{l^{2}}\left(\arctan \left(t-\frac{\eta}{l}\right)+\arctan \left(D_{l, \eta}^{-}\right)\right)\right)}{\exp \left(v^{\frac{1}{3}}\left\langle v^{\frac{1}{3}} t_{l, \xi}\right\rangle^{-(1+\beta)} \frac{\xi}{l^{2}}\left(\arctan \left(t-\frac{\xi}{l}\right)+\arctan \left(D_{l, \xi}^{-}\right)\right)\right)}-1\right| \\
& \lesssim\left|\prod_{m=E(\sqrt{|\xi|})}^{m=E(\sqrt{|\eta|})+1} \frac{1}{G_{m}(\xi)}-1\right|+\left|\prod_{m=E(\sqrt{|\eta|})}^{l+1} \frac{G_{m}(\eta)}{G_{m}(\xi)}-1\right| \\
& +\left|\frac{\exp \left(v^{\frac{1}{3}}\left\langle v^{\frac{1}{3}} t_{l, \eta}\right\rangle^{-(1+\beta)} \frac{\eta}{l^{2}} \arctan \left(D_{l, \eta}^{-}\right)\right)}{\exp \left(v^{\frac{1}{3}}\left\langle v^{\frac{1}{3}} t_{l, \xi}\right\rangle^{-(1+\beta)} \frac{\xi}{l^{2}} \arctan \left(D_{l, \xi}^{-}\right)\right)}-1\right|+\left|\frac{\exp \left(v^{\frac{1}{3}}\left\langle v^{\frac{1}{3}} t_{l, \eta}\right\rangle^{-(1+\beta)} \frac{\eta}{l^{2}}\right)}{\exp \left(v^{\frac{1}{3}}\left\langle v^{\frac{1}{3}} t_{l, \xi}\right\rangle^{-(1+\beta)} \frac{\xi}{l^{2}}\right)}-1\right| \\
& +\left|\exp \left(v^{\frac{1}{3}}\left\langle v^{\frac{1}{3}} t_{l, \eta}\right\rangle^{-(1+\beta)} \frac{\eta}{l^{2}}\left(\arctan \left(t-\frac{\eta}{l}\right)-\arctan \left(t-\frac{\xi}{l}\right)\right)\right)-1\right| .
\end{aligned}
$$

Here and in the rest of the proof we will always use the fact that for $x, y \lesssim 1$, we have $|x y-1| \lesssim|x||y-1|+|x-1| \lesssim|x-1|+|y-1|$.

Then the lemma follows from the inequalities below, which follow from (6.2) and the fact that $|f(x)-f(y)| \lesssim\left\|f^{\prime}(z)\right\|_{L^{\infty}}|x-y|$ :

$$
\begin{aligned}
& \left|\prod_{m=E(\sqrt{|\xi|})}^{m=E(\sqrt{|\eta|})+1} \frac{1}{G_{m}(\xi)}-1\right| \lesssim|\sqrt{\xi}-\sqrt{\eta}| \frac{v^{\frac{1}{3}}}{\left\langle v^{\frac{1}{3}} \eta^{\frac{1}{2}}\right\rangle^{1+\beta}} \lesssim|\eta-\xi|\langle\xi\rangle^{-1}, \\
& \left|\prod_{m=E(\sqrt{|\eta|})}^{l+1} \frac{G_{m}(\eta)}{G_{m}(\xi)}-1\right| \lesssim \exp \left(C \frac{|\xi-\eta|}{\langle\eta\rangle} \sum_{m=E(\sqrt{|\eta|})}^{l+1}\left(F_{2}^{+}+F_{2}^{-}\right)(m, \eta)\right)-1 \lesssim \frac{|\xi-\eta|}{\langle\eta\rangle},
\end{aligned}
$$


and

$$
\begin{aligned}
& \left|\frac{\exp \left(v^{\frac{1}{3}}\left\langle v^{\frac{1}{3}} t_{l, \eta}\right\rangle^{-(1+\beta)} \frac{\eta}{l^{2}} \arctan \left(D_{l, \eta}^{-}\right)\right)}{\exp \left(v^{\frac{1}{3}}\left\langle v^{\frac{1}{3}} t l, \xi\right\rangle^{-(1+\beta)} \frac{\xi}{l^{2}} \arctan \left(D_{l, \xi}^{-}\right)\right)}-1\right| \lesssim \exp \left(C \frac{|\xi-\eta|}{\langle\eta\rangle}\right)-1 \lesssim \frac{|\xi-\eta|}{\langle\eta\rangle}, \\
& \left|\frac{\exp \left(v^{\frac{1}{3}}\left\langle v^{\frac{1}{3}} t_{l, \eta}\right\rangle^{-(1+\beta)} \frac{\eta}{l^{2}}\right)}{\exp \left(v^{\frac{1}{3}}\left\langle v^{\frac{1}{3}} t_{l, \xi}\right\rangle^{-(1+\beta)} \frac{\xi}{l^{2}}\right)}-1\right| \lesssim \exp \left(C \frac{|\xi-\eta|}{\langle\eta\rangle}\right)-1 \lesssim \frac{|\xi-\eta|}{\langle\eta\rangle}, \\
& \left|\exp \left(v^{\frac{1}{3}}\left\langle v^{\frac{1}{3}} t_{l, \eta}\right\rangle^{-(1+\beta)} \frac{\eta}{l^{2}}\left(\arctan \left(t-\frac{\eta}{l}\right)-\arctan \left(t-\frac{\xi}{l}\right)\right)\right)-1\right| \\
& \quad \lesssim v^{\frac{1}{3}}\left\langle v^{\frac{1}{3}} t_{l, \eta}\right\rangle^{-(1+\beta)} \frac{\eta}{l^{2}}\left(\arctan \left(t-\frac{\eta}{l}\right)-\arctan \left(t-\frac{\xi}{l}\right)\right) \\
& \quad \lesssim v^{\frac{1}{3}}\left\langle v^{\frac{1}{3}} t_{l, \eta}\right\rangle^{-(1+\beta)} \frac{\eta}{l^{2}} \min \left\{\frac{|\xi-\eta|}{l}, 1\right\} \lesssim\left\{\begin{array}{l}
v^{-\frac{1}{3}} \frac{|\xi-\eta|}{\eta}, \quad t \approx \frac{\eta}{l} \lesssim v^{-\frac{1}{3}}, \\
v^{-\frac{1}{3} \beta} t^{1-\beta} \frac{|\xi-\eta|}{\eta}, \quad t \approx \frac{\eta}{l} \gtrsim v^{-\frac{1}{3}} .
\end{array}\right.
\end{aligned}
$$

(3b) $k \neq l,\left|t-\frac{\eta}{k}\right| \gtrsim \frac{\eta}{k^{2}}$ and $\left|t-\frac{\xi}{l}\right| \gtrsim \frac{\xi}{l^{2}}$ with $k<l$. We have

$$
\begin{aligned}
\mid w(t, \eta) & -w(t, \xi)|=w(t, \xi)| \frac{w(t, \eta)}{w(t, \xi)}-1 \mid \\
& \lesssim\left|\prod_{m=E(\sqrt{|\xi|})}^{m=E(\sqrt{|\eta|})+1} \frac{1}{G_{m}(\xi)}-1\right|+\left|\prod_{m=E(\sqrt{|\eta|})}^{\max \{l, k\}+1} \frac{G_{m}(\eta)}{G_{m}(\xi)}-1\right|+\left|\prod_{m=\max \{l, k\}}^{\min \{k, l\}+1} G_{m}(\eta)-1\right| \\
& +\left|\frac{\exp \left(v^{\frac{1}{3}}\left\langle v^{\frac{1}{3}} t_{k, \eta}\right\rangle^{-(1+\beta)} \frac{\eta}{k^{2}}\left(\arctan \left(t-\frac{\eta}{k}\right)+\arctan \left(D_{k, \eta}^{-}\right)\right)\right)}{\exp \left(v^{\frac{1}{3}}\left\langle v^{\frac{1}{3}} t_{l, \xi}\right\rangle^{-(1+\beta)} \frac{\xi}{l^{2}}\left(\arctan \left(t-\frac{\xi}{l}\right)+\arctan \left(D_{l, \xi}^{-}\right)\right)\right)}-1\right| .
\end{aligned}
$$

Let $F_{3}(t, k, \eta)=v^{\frac{1}{3}}\left\langle v^{\frac{1}{3}} t_{k, \eta}\right\rangle^{-(1+\beta)} \frac{\eta}{k^{2}}\left(\arctan \left(t-\frac{\eta}{k}\right)+\arctan \left(D_{k, \eta}^{-}\right)\right)$; we get

$$
\left|F_{3}\right| \lesssim k^{-1}, \quad\left|\partial_{k} F_{3}\right| \lesssim \frac{1}{\langle k\rangle}, \quad\left|\partial_{\eta} F_{3}\right| \lesssim \frac{1}{\langle\eta\rangle},
$$

where we used the fact that $\eta \gtrsim k^{2}$. Then the lemma follows from (6.3), (6.4) and the following two inequalities:

$$
\begin{aligned}
& \left|\frac{\exp \left(v^{\frac{1}{3}}\left\langle v^{\frac{1}{3}} t_{k, \eta}\right\rangle^{-(1+\beta)} \frac{\eta}{k^{2}}\left(\arctan \left(t-\frac{\eta}{k}\right)+\arctan \left(D_{k, \eta}^{-}\right)\right)\right)}{\exp \left(v^{\frac{1}{3}}\left\langle v^{\frac{1}{3}} t_{l, \xi}\right\rangle^{-(1+\beta)} \frac{\xi}{l^{2}}\left(\arctan \left(t-\frac{\xi}{l}\right)+\arctan \left(D_{l, \xi}^{-}\right)\right)\right)}-1\right| \\
& \lesssim e^{F_{3}(t, k, \eta)-F_{3}(t, l, \xi)}-1 \lesssim\left|F_{3}(t, k, \eta)-F_{3}(t, l, \xi)\right| \\
& \quad \lesssim \frac{1}{\langle k\rangle}|k-l|+\frac{1}{\langle\eta\rangle}|\xi-\eta| \lesssim \frac{|\xi-\eta|}{\langle\eta\rangle}
\end{aligned}
$$

and

$$
\left|\prod_{m=\max \{k, l\}}^{\min \{k, l\}+1} G_{m}(\eta)-1\right| \lesssim|l-k| \frac{\frac{v^{\frac{1}{3}} \eta}{l^{2}}}{\left\langle\frac{v^{\frac{1}{3}} \eta}{l}\right\rangle^{1+\beta}} \lesssim \frac{|k-l|}{l} \lesssim \frac{|\xi-\eta|}{\langle\eta\rangle},
$$

where we used the fact that $|k-l| \lesssim\left\langle\frac{\xi-\eta}{t}\right\rangle, l \approx \frac{\eta}{t}$. 
(3c) $|\xi-\eta| \gtrsim \frac{\xi}{l} \approx \frac{\eta}{k}$. In this case, similarly we have

$$
|w(t, \xi)-w(t, \eta)| \lesssim \frac{|\xi-\eta|}{\langle\eta\rangle} .
$$

Case 4. For $2 \eta \leq t \leq 2 \xi$, then $t \in I_{1, \xi}$ and

$$
\begin{aligned}
|w(t, \eta)-w(t, \xi)| & =w(t, \xi)\left|\frac{w(2 \eta, \eta)}{w\left(t_{l, \xi}, \xi\right) g_{l}\left(t-\frac{\xi}{l}, \xi\right)}-1\right| \\
& \lesssim\left|\frac{w(2 \eta, \eta)}{w\left(t_{l, \xi}, \xi\right) g_{l}\left(t-\frac{\xi}{l}, \xi\right)}-1\right| \\
& \lesssim\left|\prod_{m=E(\sqrt{|\xi|})}^{m=E(\sqrt{|\eta|})+1} \frac{1}{G_{m}(\xi)}-1\right|+\left|\prod_{m=E(\sqrt{|\eta|})}^{2} \frac{G_{m}(\eta)}{G_{m}(\xi)}-1\right| \\
& +\left|\frac{\exp \left(v^{\frac{1}{3}}\left\langle v^{\frac{1}{3}} t_{1, \eta}\right\rangle^{-(1+\beta)} \eta\left(\arctan (\eta)+\arctan \left(\frac{1}{3} \eta\right)\right)\right)}{\exp \left(v^{\frac{1}{3}}\left\langle v^{\frac{1}{3}} t_{1, \xi}\right\rangle^{-(1+\beta)} \xi\left(\arctan (t-\xi)+\arctan \left(\frac{1}{3} \xi\right)\right)\right)}-1\right| .
\end{aligned}
$$

Thus, the lemma follows from (6.3), (6.4) and the following inequalities:

$$
\begin{aligned}
& \left|\exp \left(v^{\frac{1}{3}}\left\langle v^{\frac{1}{3}} t_{1, \xi}\right\rangle^{-(1+\beta)} \xi\left(\arctan (t-\xi)+\arctan \left(D_{1, \xi}^{-}\right)\right)\right)-1\right| \\
& \lesssim \mid v^{\frac{1}{3}}\left\langle v^{\frac{1}{3}} t_{1, \xi}\right\rangle^{-(1+\beta)} \xi\left(\arctan (t-\xi)+\arctan \left(\frac{1}{3} \xi\right)\right) \\
& \quad-\left(v^{\frac{1}{3}}\left\langle v^{\frac{1}{3}} t_{1, \eta}\right\rangle^{-(1+\beta)} \eta\left(\arctan (\eta)+\arctan \left(\frac{1}{3} \eta\right)\right)\right) \mid \\
& \lesssim|\arctan (t-\xi)-\arctan (\eta)|+|\xi-\eta|\langle\xi\rangle^{-1} \\
& \lesssim \max \{\arctan (\xi)-\arctan (\eta), \arctan (\eta)-\arctan (2 \eta-\xi)\}+|\xi-\eta|\langle\xi\rangle^{-1} \\
& \lesssim|\xi-\eta|\langle\xi\rangle^{-1} .
\end{aligned}
$$

Case 5. For $t \geq 2 \xi$. We get by (6.3) and (6.4) that

$$
\begin{aligned}
|w(2 \eta, \eta)-w(2 \xi, \xi)| & =w(2 \xi, \xi)\left|\frac{w(2 \eta, \eta)}{w(2 \xi, \xi)}-1\right| \\
& \lesssim\left|\prod_{m=E(\sqrt{|\xi|})}^{m=E(\sqrt{|\eta|})+1} \frac{1}{G_{m}(\xi)}-1\right|+\left|\prod_{m=E(\sqrt{|\eta|})}^{1} \frac{G_{m}(\eta)}{G_{m}(\xi)}-1\right| \\
& \lesssim|\xi-\eta|\langle\xi\rangle^{-1}
\end{aligned}
$$

Thus we have proved Lemma 6.1.

\section{Remainder}

In this section we deal with the remainder and prove Proposition 2.7. Now, the commutator cannot gain us anything so we may as well treat each term separately. We rewrite both 
terms on the Fourier side:

$$
\begin{aligned}
\mathcal{R}= & \sum_{N \in \mathbb{D}} \sum_{N^{\prime} \approx N} \sum_{k, l} \int_{\eta, \xi} A^{\sigma} \overline{\hat{f}}_{k}(\eta) A_{k}^{\sigma}(\eta) \hat{u}_{l}(\xi)_{N} \widehat{\nabla f}_{k-l}(\eta-\xi)_{N^{\prime}} d \eta d \xi \\
& +\sum_{N \in \mathbb{D}} \sum_{N^{\prime} \approx N} \sum_{k, l} \int_{\eta, \xi} A^{\sigma} \overline{\hat{f}}_{k}(\eta) \hat{u}_{l}(\xi)_{N} A_{k-l}^{\sigma}(\eta-\xi) \widehat{\nabla f}_{k-l}(\eta-\xi)_{N^{\prime}} d \eta d \xi
\end{aligned}
$$

On the support of the integrand, $|l, \xi| \approx|k-l, \eta-\xi|$; thus

$$
A_{k}^{\sigma}(\eta) \approx\langle k, \eta\rangle^{\sigma} \lesssim\langle l, \xi\rangle^{\sigma}+\langle k-l, \eta-\xi\rangle^{\sigma} \approx\langle l, \xi\rangle\langle k-l, \eta-\xi\rangle^{\sigma-1} \approx A_{k-l}^{\sigma}(\eta-\xi),
$$

which implies that

$$
|\mathcal{R}| \lesssim \sum_{N \in \mathbb{D}}\left\|A^{\sigma} f\right\|_{2}\left\|u_{N}\right\|_{H^{3}}\left\|f_{\sim N}\right\|_{H^{\sigma}} .
$$

Therefore we get by (4.4),

$$
|\mathcal{R}| \lesssim\|f\|_{H^{\sigma}}^{2}\|u\|_{H^{3}} \lesssim \frac{\varepsilon v^{\frac{1}{3}}}{\left\langle t^{2}\right\rangle}\left\|A^{\sigma} f\right\|_{2}^{2},
$$

which gives Proposition 2.7 .

\section{Reaction}

In this section we deal with the reaction term and prove Proposition 2.8. We focus first on an individual frequency shell and divide each into several natural pieces:

$$
R_{N}=R_{N}^{1}+R_{N}^{\varepsilon, 1}+R_{N}^{2}+R_{N}^{3}
$$

where

$$
\begin{aligned}
R_{N}^{1} & =\sum_{k, l \neq 0} \int_{\eta, \xi} A^{\sigma} \overline{\hat{f}}_{k}(\eta) A_{k}^{\sigma}(\eta)(\eta l-\xi k) \widehat{\phi}_{l}(\xi)_{N} \hat{f}_{k-l}(\eta-\xi)_{<N / 8} d \eta d \xi, \\
R_{N}^{\varepsilon, 1} & =-\sum_{k, l \neq 0} \int_{\eta, \xi} A^{\sigma} \overline{\hat{f}}_{k}(\eta) A_{k}^{\sigma}(\eta)\left[\widehat{\left(1-v^{\prime}\right) \nabla^{\perp} \phi_{l}}\right](\xi)_{N} \cdot \widehat{\nabla f}_{k-l}(\eta-\xi)_{<N / 8} d \eta d \xi, \\
R_{N}^{2} & =\sum_{k} \int_{\eta, \xi} A^{\sigma} \overline{\hat{f}}_{k}(\eta) A_{k}^{\sigma}(\eta) \hat{g}(\xi)_{N}{\widehat{\partial f_{v} f}}_{k}(\eta-\xi)_{<N / 8} d \eta d \xi, \\
R_{N}^{3} & =-\sum_{k, l} \int_{\eta, \xi} A^{\sigma} \overline{\hat{f}}_{k}(\eta) A_{k-l}^{\sigma}(\eta-\xi) \hat{u}_{l}(\xi)_{N} \widehat{\nabla f}_{k-l}(\eta-\xi)_{<N / 8} d \eta d \xi .
\end{aligned}
$$

\subsection{Main contribution}

The main contribution comes from $R_{N}^{1}$. We subdivide this integral depending on whether $(l, \xi)$ and/or $(k, \eta)$ are resonant, as each combination requires slightly different treatment. 
Define the partition

$$
\begin{aligned}
1 & =1_{t \notin I_{k, \eta}, t \notin I_{l, \xi}}+1_{t \notin I_{k, \eta}, t \in I_{l, \xi}}+1_{t \in I_{k, \eta}, t \notin I_{l, \xi}}+1_{t \in I_{k, \eta}, t \in I_{l, \xi}} \\
& =\chi^{\mathrm{NR}, \mathrm{NR}}+\chi^{\mathrm{NR}, \mathrm{R}}+\chi^{\mathrm{R}, \mathrm{NR}}+\chi^{\mathrm{R}, \mathrm{R}},
\end{aligned}
$$

where the NR and R denote "nonresonant" and "resonant" respectively, referring to $(k, \eta)$ and $(l, \xi)$. Correspondingly, denote

$$
\begin{aligned}
& R_{N}^{1}=\underbrace{\sum_{l \neq 0} \int_{\eta, \xi} \chi^{D} A^{\sigma} \overline{\hat{f}}_{l}(\eta) A_{l}^{\sigma}(\eta)(\eta l-\xi l) \hat{\phi}_{l}(\xi)_{N} \hat{f}_{0}(\eta-\xi)_{<N / 8} d \eta d \xi}_{R_{N, D}} \\
& +\sum_{l \neq 0} \int_{\eta, \xi}\left[\chi^{\mathrm{NR}, \mathrm{NR}}+\chi^{\mathrm{NR}, \mathrm{R}}+\chi^{\mathrm{R}, \mathrm{NR}}+\chi^{\mathrm{R}, \mathrm{R}}\right]\left(1-\chi^{D}\right) \\
& \times \frac{A^{\sigma} \overline{\hat{f}}_{l}(\eta) A_{l}^{\sigma}(\eta)(\eta l-\xi l) \hat{\phi}_{l}(\xi)_{N} \hat{f}_{0}(\eta-\xi)_{<N / 8} d \eta d \xi}{R_{N,=}^{\mathrm{NR}, \mathrm{NR}}+R_{N,=}^{\mathrm{NR}, \mathrm{R}}+R_{N,=}^{\mathrm{R}, \mathrm{NR}}+R_{N,=}^{\mathrm{R}, \mathrm{R}}} \\
& +\underbrace{\sum_{k, l \neq 0, k \neq l} \int_{\eta, \xi}\left(1-\chi^{D_{1}}\right) A^{\sigma} \overline{\hat{f}}_{k}(\eta) A_{k}^{\sigma}(\eta)(\eta l-\xi k) \hat{\phi}_{l}(\xi)_{N} \hat{f}_{k-l}(\eta-\xi)_{<N / 8} d \eta d \xi}_{R_{N, \neq, *}} \\
& +\sum_{k, l \neq 0, k \neq l} \int_{\eta, \xi}\left[\chi^{\mathrm{NR}, \mathrm{NR}}+\chi^{\mathrm{NR}, \mathrm{R}}+\chi^{\mathrm{R}, \mathrm{NR}}+\chi^{\mathrm{R}, \mathrm{R}}\right] \chi^{D_{1}} \\
& \frac{\times A^{\sigma} \overline{\hat{f}}_{k}(\eta) A_{k}^{\sigma}(\eta)(\eta l-\xi k) \hat{\phi}_{l}(\xi)_{N} \hat{f}_{k-l}(\eta-\xi)_{<N / 8} d \eta d \xi}{R_{N}^{\mathrm{NR}, \mathrm{NR}}+R_{N}^{\mathrm{NR}, \mathrm{R}}+R_{N}^{\mathrm{R}, \mathrm{NR}}+R_{N}^{\mathrm{R}, \mathrm{R}}} \\
& =R_{N, D}+R_{N,=}^{\mathrm{NR}, \mathrm{NR}}+R_{N,=}^{\mathrm{NR}, \mathrm{R}}+R_{N,=}^{\mathrm{R}, \mathrm{NR}}+R_{N,=}^{\mathrm{R}, \mathrm{R}} \\
& +R_{N, \neq, *}+R_{N}^{\mathrm{NR}, \mathrm{NR}}+R_{N}^{\mathrm{NR}, \mathrm{R}}+R_{N}^{\mathrm{R}, \mathrm{NR}}+R_{N}^{\mathrm{R}, \mathrm{R}},
\end{aligned}
$$

where $\chi^{D}$ is a characteristic function (the indicator function) of the set

$$
D=\left\{(l, \xi):|l| \geq \frac{5}{4}|\xi|\right\}
$$

and $\chi^{D_{1}}$ is a characteristic function (the indicator function) of the set

$$
D_{1}=\left\{(l, k, \xi, \eta):|l| \leq|\xi|,|l-k, \xi-\eta| \leq \frac{1}{1000}|l, \xi|\right\},
$$

8.1.1. Treatment of $\boldsymbol{R}_{N, \boldsymbol{D}}$. For the case $|l| \geq \frac{5}{4}|\xi|$, we get for $t \geq 1$,

$$
\frac{A_{l}^{\sigma}(\eta)|l|}{l^{2}+|l t-\xi|^{2}} \lesssim \frac{A_{l}^{\sigma}(\xi)|l|}{l^{2}+t^{2} l^{2}} \lesssim \frac{|l|^{\sigma-1}}{1+t^{2}},
$$

which implies that

$$
\begin{aligned}
\left|R_{N, D}\right| & \lesssim \frac{1}{\left\langle t^{2}\right\rangle}\left\|A^{\sigma} f_{\sim N}\right\|_{L^{2}}\left\|\left\langle\partial_{z}\right\rangle^{\sigma-1} \Delta_{L} \Delta_{t}^{-1} P_{\neq} f_{N}\right\|_{L^{2}}\left\|f_{0}\right\|_{H^{3}} \\
& \lesssim \frac{\varepsilon v^{\frac{1}{3}}}{\left\langle t^{2}\right\rangle}\left\|A^{\sigma} f\right\|_{2}^{2} .
\end{aligned}
$$


In the next four subsections we will use the fact that for $|l| \leq \frac{5}{4}|\xi|$ and $|\xi-\eta| \leq$ $\frac{3}{8}|l, \xi| \leq \frac{27}{40}|\xi|$, we have $|\eta| \geq \frac{13}{40}|\xi| \geq \frac{13}{50}|l|$, which gives that

$$
w_{l}(t, \eta)=w(t, \eta), \quad w_{l}(t, \xi)=w(t, \xi) .
$$

8.1.2. Treatment of the zero mode $R_{N,=}^{N R, N R}$. We have

$$
\begin{aligned}
R_{N,=}^{\mathrm{NR}, \mathrm{NR}} \lesssim \sum_{l \neq 0} \int_{\eta, \xi} & \chi^{\mathrm{NR}, \mathrm{NR}}\left(1-\chi^{D}\right) \\
& \times\left|A^{\sigma} \overline{\hat{f}}_{l}(\eta) \frac{A_{l}^{\sigma}(\eta)|l|}{l^{2}+|l t-\xi|^{2}} \widehat{\Delta_{L} \Delta_{t}^{-1}} f_{l}(\xi)_{N}{\widehat{\partial f_{v}}}_{0}(\eta-\xi)_{<N / 8}\right| d \eta d \xi
\end{aligned}
$$

According to the relation between $t$ and $\xi$, we have the following three cases.

Case 1: $t \leq \max \{t(\xi), t(\eta)\} \approx \sqrt{|\xi|} \approx \sqrt{N}$. Then in this case,

$$
\frac{A_{l}^{\sigma}(\eta)|l|}{l^{2}+|l t-\xi|^{2}} \lesssim \begin{cases}\frac{A_{l}^{\sigma}(\xi)|l|}{l^{2}\left(1+\frac{|\xi|^{2}}{l^{4}}\right)} \lesssim \frac{A_{l}^{\sigma}(\xi)}{\sqrt{\xi}} & \text { if }|l| \leq E(\sqrt{\xi}), \\ \frac{A_{l}^{\sigma}(\xi)}{\sqrt{\xi}} & \text { if }|l| \geq E(\sqrt{\xi})+1,\end{cases}
$$

which implies

$$
\begin{aligned}
\left|R_{N,=}^{\mathrm{NR}, \mathrm{NR}}\right| & \lesssim \frac{1}{\langle t\rangle}\left\|A^{\sigma} f_{\sim N}\right\|_{L^{2}}\left\|A^{\sigma} \Delta_{L} \Delta_{t}^{-1} P_{\neq} f_{N}\right\|_{L^{2}}\left\|\partial_{v} f_{0}\right\|_{H^{3}} \\
& \lesssim \frac{\varepsilon v^{\frac{1}{3}}}{\left\langle v^{\frac{1}{2}} t^{\frac{1}{2}}\right\rangle\langle t\rangle}\left\|A^{\sigma} f_{\sim N}\right\|_{L^{2}}\left\|A^{\sigma} \Delta_{L} \Delta_{t}^{-1} P_{\neq} f_{N}\right\|_{L^{2}} .
\end{aligned}
$$

Case 2: $t \geq 2|\xi|$ or $t \geq 2|\eta|$. Then in this case,

$$
\frac{|l|}{l^{2}+|l t-\xi|^{2}} \lesssim \frac{1}{1+t^{2}}
$$

and

$$
\begin{aligned}
\frac{|l|}{l^{2}+|l t-\xi|^{2}} & \lesssim \frac{|l|}{l^{2}+|l t-\xi|^{2}}-\frac{|l|}{l^{2}+|l t-\eta|^{2}}+\frac{|l|}{l^{2}+|l t-\eta|^{2}} \\
& \lesssim \frac{|l||\eta-\xi|(2|\xi-l t|+|\xi-\eta|)}{\left(l^{2}+|l t-\eta|^{2}\right)\left(l^{2}+|l t-\xi|^{2}\right)}+\frac{|l|}{l^{2}+|l t-\eta|^{2}} \\
& \lesssim \frac{|l|\langle\xi-\eta\rangle^{2}}{l^{2}+|l t-\eta|^{2}} \lesssim \frac{\langle\xi-\eta\rangle^{2}}{1+t^{2}},
\end{aligned}
$$

which implies that

$$
\begin{aligned}
\left|R_{N,=}^{\mathrm{NR}, \mathrm{NR}}\right| & \lesssim \frac{1}{\left\langle t^{2}\right\rangle}\left\|A^{\sigma} f_{\sim N}\right\|_{L^{2}}\left\|A^{\sigma} \Delta_{L} \Delta_{t}^{-1} P_{\neq} f_{N}\right\|_{L^{2}}\left\|f_{0}\right\|_{H^{5}} \\
& \lesssim \frac{\varepsilon v^{\frac{1}{3}}}{\left\langle t^{2}\right\rangle}\left\|A^{\sigma} f_{\sim N}\right\|_{L^{2}}\left\|A^{\sigma} \Delta_{L} \Delta_{t}^{-1} P_{\neq} f_{N}\right\|_{L^{2}} .
\end{aligned}
$$


Case 3: $t \in I_{t}(\xi) \cap I_{t}(\eta)$. In this case there exists $k, l^{\prime}$ so that $t \in I_{k, \eta} \cap I_{l^{\prime}, \xi}$. By Lemma 3.2, we get $k \approx l^{\prime}$.

If $l \leq \frac{1}{2} \min \left\{\left|l^{\prime}\right|,|k|\right\}$, then

$$
\begin{aligned}
\frac{|l|}{l^{2}\left(1+\left|t-\frac{\xi}{l}\right|^{2}\right)} & \lesssim \sqrt{\frac{|l|}{l^{2}\left(1+\left|t-\frac{\eta}{l}\right|^{2}\right)}} \sqrt{\frac{|l|}{l^{2}\left(1+\left|t-\frac{\xi}{l}\right|^{2}\right)}}\langle\xi-\eta\rangle \\
& \lesssim \frac{\langle\xi-\eta\rangle}{l\left(1+\frac{\xi^{2}}{l^{2}}\right)} \lesssim \frac{\langle\xi-\eta\rangle}{1+\frac{\xi^{2}}{l^{2}}} \lesssim \frac{\langle\xi-\eta\rangle}{1+t^{2}},
\end{aligned}
$$

where here we use the fact that $t \approx \frac{\xi}{l^{\prime}}$.

If $l>\frac{1}{2} \min \left\{\left|l^{\prime}\right|,|k|\right\}$, then

$$
\frac{1}{l^{2}} \lesssim \frac{1}{\min \left\{\left|l^{\prime}\right|,|k|\right\}^{2}} \lesssim \frac{1}{\left|l^{\prime} k\right|}
$$

and by the fact that for $t \in I_{l^{\prime}, \xi} \cap I_{k, \eta}$ and $t \notin I_{l, \xi} \cup I_{l, \eta}$, it holds that $\left|t-\frac{\eta}{l}\right| \geq\left|t-\frac{\eta}{k}\right|$ and $\left|t-\frac{\xi}{l}\right| \geq\left|t-\frac{\xi}{l^{\prime}}\right|$. Thus, by (8.2) we get

$$
\begin{aligned}
\frac{|l|\left\langle v^{\frac{1}{2}}\left(\frac{\xi}{k}\right)^{\frac{1}{2}}\right\rangle^{-1}}{l^{2}\left(1+\left|t-\frac{\xi}{l}\right|^{2}\right)} & \lesssim \sqrt{\frac{|l|}{l^{2}\left(1+\left|t-\frac{\eta}{l}\right|^{2}\right)}} \sqrt{\frac{|l|}{l^{2}\left(1+\left|t-\frac{\xi}{l}\right|^{2}\right)}}\langle\xi-\eta\rangle\left\langle v^{\frac{1}{2}}\left(\frac{\xi}{k}\right)^{\frac{1}{2}}\right\rangle^{-1} \\
& \lesssim \sqrt{\frac{1}{k\left(1+\left|t-\frac{\eta}{k}\right|^{2}\right)}} \sqrt{\frac{1}{l^{\prime}\left(1+\left|t-\frac{\xi}{l^{\prime}}\right|^{2}\right)}}\langle\xi-\eta\rangle\left\langle v^{\left.\frac{1}{2}\left(\frac{\xi}{k}\right)^{\frac{1}{2}}\right\rangle^{-1}}\right. \\
& \lesssim \sqrt{\frac{\eta}{k^{2}\left(1+\left|t-\frac{\eta}{k}\right|^{2}\right)}} \sqrt{\frac{\xi}{l^{\prime 2}\left(1+\left|t-\frac{\xi}{l^{\prime}}\right|^{2}\right)}}\langle\xi-\eta\rangle\left\langle v^{\frac{1}{3}}\left(\frac{\xi}{k}\right)\right\rangle^{-(1+\beta)} \\
& \lesssim \sqrt{\frac{\partial_{t} w(t, \eta)}{w(t, \eta)} \sqrt{\frac{\partial_{t} w(t, \xi)}{w(t, \xi)}}\langle\xi-\eta\rangle v^{-\frac{1}{3}}} .
\end{aligned}
$$

In the third inequality we use the fact that for $0<\beta \leq \frac{1}{2}$,

$$
\left\langle v^{\frac{1}{2}}\left(\frac{\xi}{k}\right)^{\frac{1}{2}}\right\rangle^{-1} \lesssim \frac{\xi}{k}\left\langle v^{\frac{1}{3}}\left(\frac{\xi}{k}\right)\right\rangle^{-(1+\beta)} .
$$

Thus, by the fact that $t \approx \frac{\xi}{k}$ we get

$$
\begin{aligned}
& \left|R_{N,=}^{\mathrm{NR}, \mathrm{NR}}\right| \lesssim \frac{1}{\left\langle t^{2}\right\rangle}\left\|A^{\sigma} f_{\sim N}\right\|_{L^{2}}\left\|A^{\sigma} \Delta_{L} \Delta_{t}^{-1} f_{N}\right\|_{L^{2}}\left\|f_{0}\right\|_{H^{4}} \\
& +\left\|\sqrt{\frac{\partial_{t} w}{w}} A^{\sigma} f_{\sim N}\right\|_{L^{2}}\left\|\sqrt{\frac{\partial_{t} w}{w}} \chi_{R} A^{\sigma} \Delta_{L} \Delta_{t}^{-1} f_{N}\right\|_{L^{2}}\left\|v^{-\frac{1}{3}}\left(v^{\frac{1}{2}} t^{\frac{1}{2}} \partial_{v}\right) f_{0}\right\|_{H^{3}} \\
& \lesssim \frac{\varepsilon \mathcal{V}^{\frac{1}{3}}}{\left\langle t^{2}\right\rangle}\left\|A^{\sigma} f_{\sim N}\right\|_{L^{2}}\left\|A^{\sigma} \Delta_{L} \Delta_{t}^{-1} f_{N}\right\|_{L^{2}} \\
& +\varepsilon\left\|\sqrt{\frac{\partial_{t} w}{w}} A^{\sigma} f_{\sim N}\right\|_{L^{2}}\left\|\sqrt{\frac{\partial_{t} w}{w}} \chi_{R} A^{\sigma} \Delta_{L} \Delta_{t}^{-1} P_{\neq} f_{N}\right\|_{L^{2}} .
\end{aligned}
$$


Putting together all the above estimates, we conclude that

$$
\begin{aligned}
\left|R_{N,=}^{\mathrm{NR}, \mathrm{NR}}\right| \lesssim & \frac{\varepsilon v^{\frac{1}{3}}}{\left\langle v^{\frac{1}{2}} t^{\frac{3}{2}}\right\rangle}\left\|A^{\sigma} f_{\sim N}\right\|_{L^{2}}\left\|A^{\sigma} \Delta_{L} \Delta_{t}^{-1} f_{N}\right\|_{L^{2}} \\
& +\frac{\varepsilon v^{\frac{1}{3}}}{\left\langle t^{2}\right\rangle}\left\|A^{\sigma} f_{\sim N}\right\|_{L^{2}}\left\|A^{\sigma} \Delta_{L} \Delta_{t}^{-1} f_{N}\right\|_{L^{2}} \\
& +\varepsilon\left\|\sqrt{\frac{\partial_{t} w}{w}} A^{\sigma} f_{\sim N}\right\|_{L^{2}}\left\|\sqrt{\frac{\partial_{t} w}{w}} \chi_{R} A^{\sigma} \Delta_{L} \Delta_{t}^{-1} P_{\neq} f_{N}\right\|_{L^{2}} .
\end{aligned}
$$

8.1.3. Treatment of the zero mode $\boldsymbol{R}_{N,=}^{\mathbf{N R}, \mathbf{R}}$. Since $t \in I_{l, \xi}$, if $t \leq t(\eta) \approx \sqrt{|\xi|} \approx \sqrt{N}$, then $l \approx \sqrt{|\xi|}$; thus by the fact that

$$
\frac{A_{l}^{\sigma}(\eta)|l|}{l^{2}+|l t-\xi|^{2}} \lesssim \frac{A_{l}^{\sigma}(\xi)}{|l|} \lesssim \frac{A_{l}^{\sigma}(\xi)}{\sqrt{N}},
$$

we obtain

$$
\begin{aligned}
\left|R_{N,=}^{\mathrm{NR}, \mathrm{R}}\right| & \lesssim \frac{1}{\langle t\rangle}\left\|A^{\sigma} f_{\sim N}\right\|_{L^{2}}\left\|A^{\sigma} \Delta_{L} \Delta_{t}^{-1} f_{N}\right\|_{L^{2}}\left\|\partial_{v} f_{0}\right\|_{H^{3}} \\
& \lesssim \frac{\varepsilon v^{\frac{1}{3}}}{\left\langle v^{\frac{1}{2}} t^{\frac{3}{2}}\right\rangle}\left\|A^{\sigma} f_{\sim N}\right\|_{L^{2}}\left\|A^{\sigma} \Delta_{L} \Delta_{t}^{-1} f_{N}\right\|_{L^{2}}
\end{aligned}
$$

If $t \geq 2|\eta|$, then we use the same argument as (8.4) and (8.5) and get

$$
\frac{|l| A_{k}^{\sigma}(\eta)}{l^{2}+(\xi-l t)^{2}} \lesssim \frac{\langle\xi-\eta\rangle^{2}}{\langle t\rangle^{2}} A_{l}^{\sigma}(\xi) .
$$

Therefore we get

$$
\begin{aligned}
\left|R_{N,=}^{\mathrm{NR}, \mathrm{R}}\right| & \lesssim \frac{1}{\left\langle t^{2}\right\rangle}\left\|A^{\sigma} f_{\sim N}\right\|_{L^{2}}\left\|A^{\sigma} \Delta_{L} \Delta_{t}^{-1} f_{N}\right\|_{L^{2}}\left\|f_{0}\right\|_{H^{5}} \\
& \lesssim \frac{\varepsilon v^{\frac{1}{3}}}{\left\langle t^{2}\right\rangle}\left\|A^{\sigma} f_{\sim N}\right\|_{L^{2}}\left\|A^{\sigma} \Delta_{L} \Delta_{t}^{-1} f_{N}\right\|_{L^{2}} .
\end{aligned}
$$

If $t \in I_{t}(\eta)$, then there is $k \neq l$ such that $t \in I_{k, \eta} \cap I_{l, \xi}$. By the fact that for $t \in I_{k, \eta}$ and $t \notin I_{l, \eta},\left|t-\frac{\eta}{l}\right|>\left|t-\frac{\eta}{k}\right|$, then we get by (8.6) that

$$
\begin{aligned}
& \frac{|l|\left\langle v^{\frac{1}{2}}\left(\frac{\xi}{k}\right)^{\frac{1}{2}}\right\rangle^{-1}}{l^{2}\left(1+\left|t-\frac{\xi}{l}\right|^{2}\right)} \lesssim \sqrt{\frac{|l|}{l^{2}\left(1+\left|t-\frac{\eta}{l}\right|^{2}\right)}} \sqrt{\frac{|l|}{l^{2}\left(1+\left|t-\frac{\xi}{l}\right|^{2}\right)}}\langle\xi-\eta\rangle\left\langle v^{\frac{1}{2}}\left(\frac{\xi}{k}\right)^{\frac{1}{2}}\right\rangle^{-1} \\
& \lesssim \sqrt{\frac{1}{k\left(1+\left|t-\frac{\eta}{k}\right|^{2}\right)}} \sqrt{\frac{1}{l\left(1+\left|t-\frac{\xi}{l}\right|^{2}\right)}}\langle\xi-\eta\rangle\left\langle v^{\left.\frac{1}{2}\left(\frac{\xi}{k}\right)^{\frac{1}{2}}\right\rangle^{-1}}\right. \\
& \lesssim \sqrt{\frac{\eta}{k^{2}\left(1+\left|t-\frac{\eta}{k}\right|^{2}\right)}} \sqrt{\frac{\xi}{l^{2}\left(1+\left|t-\frac{\xi}{l}\right|^{2}\right)}}\langle\xi-\eta\rangle\left\langle v^{\frac{1}{3}}\left(\frac{\xi}{k}\right)\right\rangle^{-(1+\beta)} \\
& \lesssim \sqrt{\frac{\partial_{t} w(t, \eta)}{w(t, \eta)}} \sqrt{\frac{\partial_{t} w(t, \xi)}{w(t, \xi)}}\langle\xi-\eta\rangle v^{-\frac{1}{3}}
\end{aligned}
$$


Therefore, we get

$$
\begin{aligned}
\left|R_{N,=}^{\mathrm{NR}, \mathrm{R}}\right| & \lesssim\left\|\sqrt{\frac{\partial_{t} w}{w}} A^{\sigma} f_{\sim N}\right\|\left\|_{L^{2}}\right\| \sqrt{\frac{\partial_{t} w}{w}} A^{\sigma} \chi_{R} \Delta_{L} \Delta_{t}^{-1} f_{N}\left\|_{L^{2}}\right\| v^{-\frac{1}{3}}\left(v^{\frac{1}{2}} t^{\frac{1}{2}} \partial_{v}\right) f_{0} \|_{H^{3}} \\
& \lesssim \varepsilon\left\|\sqrt{\frac{\partial_{t} w}{w}} A^{\sigma} f_{\sim N}\right\|_{L^{2}}\left\|\sqrt{\frac{\partial_{t} w}{w}} A^{\sigma} \chi_{R} \Delta_{L} \Delta_{t}^{-1} f_{N}\right\|_{L^{2}} .
\end{aligned}
$$

Putting together all the above estimates, we conclude that

$$
\begin{aligned}
\left|R_{N,=}^{\mathrm{NR}, \mathrm{R}}\right| \lesssim & \frac{\varepsilon v^{\frac{1}{3}}}{\left\langle v^{\frac{1}{2}} t^{\frac{3}{2}}\right\rangle}\left\|A^{\sigma} f_{\sim N}\right\|_{L^{2}}\left\|A^{\sigma} \Delta_{L} \Delta_{t}^{-1} f_{N}\right\|_{L^{2}} \\
& +\frac{\varepsilon \nu^{\frac{1}{3}}}{\left\langle t^{2}\right\rangle}\left\|A^{\sigma} f_{\sim N}\right\|_{L^{2}}\left\|A^{\sigma} \Delta_{L} \Delta_{t}^{-1} f_{N}\right\|_{L^{2}} \\
& +\varepsilon\left\|\sqrt{\frac{\partial_{t} w}{w}} A^{\sigma} f_{\sim N}\right\|_{L^{2}}\left\|\sqrt{\frac{\partial_{t} w}{w}} \chi_{R} A^{\sigma} \Delta_{L} \Delta_{t}^{-1} f_{N}\right\|_{L^{2}} .
\end{aligned}
$$

8.1.4. Treatment of the zero mode $\boldsymbol{R}_{N,=}^{\mathbf{R}, \mathbf{N R}}$. Since $t \in I_{l, \eta}$, if $t \leq t(\xi) \approx \sqrt{|\xi|}$, then $l \approx \sqrt{|\xi|}$. By using (8.3), we get

$$
\begin{aligned}
\left|R_{N,=}^{\mathrm{R}, \mathrm{NR}}\right| & \lesssim \frac{1}{\left\langle t^{2}\right\rangle}\left\|A^{\sigma} f_{\sim N}\right\|_{L^{2}}\left\|A^{\sigma} \Delta_{L} \Delta_{t}^{-1} f_{N}\right\|_{L^{2}}\left\|f_{0}\right\|_{H^{5}} \\
& \lesssim \frac{\varepsilon v^{\frac{1}{3}}}{\left\langle t^{2}\right\rangle}\left\|A^{\sigma} f_{\sim N}\right\|_{L^{2}}\left\|A^{\sigma} \Delta_{L} \Delta_{t}^{-1} f_{N}\right\|_{L^{2}} .
\end{aligned}
$$

Similarly if $t \geq 2|\xi|$, then by (8.5), we get

$$
\begin{aligned}
\left|R_{N,=}^{\mathrm{R}, \mathrm{NR}}\right| & \lesssim \frac{1}{\left\langle t^{2}\right\rangle}\left\|A^{\sigma} f_{\sim N}\right\|_{L^{2}}\left\|A^{\sigma} \Delta_{L} \Delta_{t}^{-1} f_{N}\right\|_{L^{2}}\left\|f_{0}\right\|_{H^{5}} \\
& \lesssim \frac{\varepsilon v^{\frac{1}{3}}}{\left\langle t^{2}\right\rangle}\left\|A^{\sigma} f_{\sim N}\right\|_{L^{2}}\left\|A^{\sigma} \Delta_{L} \Delta_{t}^{-1} f_{N}\right\|_{L^{2}} .
\end{aligned}
$$

If $t \in I_{t}(\xi)$, then there is $k \neq l$ such that $t \in I_{k, \xi} \cap I_{l, \eta}$. By the fact that for $t \in I_{k, \xi}$ and $t \notin I_{l, \xi},\left|t-\frac{\xi}{l}\right|>\left|t-\frac{\xi}{k}\right|$, then we get by (8.6) that

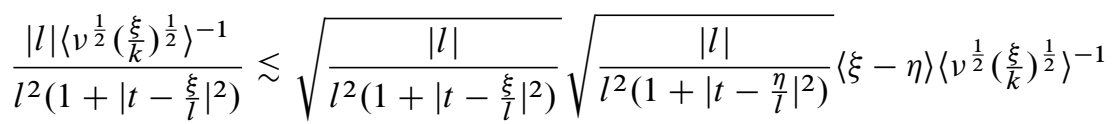

$$
\begin{aligned}
& \lesssim \sqrt{\frac{1}{k\left(1+\left|t-\frac{\xi}{k}\right|^{2}\right)}} \sqrt{\frac{1}{l\left(1+\left|t-\frac{\eta}{l}\right|^{2}\right)}}\langle\xi-\eta\rangle\left\langle v^{\left.\frac{1}{2}\left(\frac{\xi}{k}\right)^{\frac{1}{2}}\right\rangle^{-1}}\right. \\
& \lesssim \sqrt{\frac{\xi}{k^{2}\left(1+\left|t-\frac{\xi}{k}\right|^{2}\right)}} \sqrt{\frac{\eta}{l^{2}\left(1+\left|t-\frac{\eta}{l}\right|^{2}\right)}}\langle\xi-\eta\rangle\left\langle v^{\frac{1}{3}}\left(\frac{\xi}{k}\right)\right\rangle^{-(1+\beta)} \\
& \lesssim \sqrt{\frac{\partial_{t} w(t, \eta)}{w(t, \eta)}} \sqrt{\frac{\partial_{t} w(t, \xi)}{w(t, \xi)}}\langle\xi-\eta\rangle v^{-\frac{1}{3}} \text {. }
\end{aligned}
$$


Therefore, we get

$$
\begin{aligned}
\left|R_{N,=}^{\mathrm{R}, \mathrm{NR}}\right| & \lesssim\left\|\sqrt{\frac{\partial_{t} w}{w}} A^{\sigma} f_{\sim N}\right\|\left\|_{L^{2}}\right\| \sqrt{\frac{\partial_{t} w}{w}} \chi_{R} A^{\sigma} \Delta_{L} \Delta_{t}^{-1} f_{N}\left\|_{L^{2}}\right\| v^{-\frac{1}{3}}\left(v^{\frac{1}{2}} t^{\frac{1}{2}} \partial_{v}\right) f_{0} \|_{H^{3}} \\
& \lesssim \varepsilon\left\|\sqrt{\frac{\partial_{t} w}{w}} A^{\sigma} f_{\sim N}\right\|_{L^{2}}\left\|\sqrt{\frac{\partial_{t} w}{w}} \chi_{R} A^{\sigma} \Delta_{L} \Delta_{t}^{-1} f_{N}\right\|_{L^{2}} .
\end{aligned}
$$

Putting together all the above estimates, we conclude that

$$
\begin{aligned}
\left|R_{N,=}^{\mathrm{R}, \mathrm{NR}}\right| \lesssim & \frac{\varepsilon v^{\frac{1}{3}}}{\left\langle t^{2}\right\rangle}\left\|A^{\sigma} f_{\sim N}\right\|_{L^{2}}\left\|A^{\sigma} \Delta_{L} \Delta_{t}^{-1} f_{N}\right\|_{L^{2}} \\
& +\varepsilon\left\|\sqrt{\frac{\partial_{t} w}{w}} A^{\sigma} f_{\sim N}\right\|\left\|_{L^{2}}\right\| \sqrt{\frac{\partial_{t} w}{w}} \chi_{R} A^{\sigma} \Delta_{L} \Delta_{t}^{-1} f_{N} \|_{L^{2}} .
\end{aligned}
$$

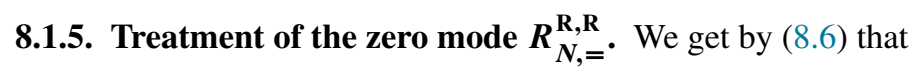

$$
\begin{aligned}
\frac{|l|\left\langle v^{\frac{1}{2}}\left(\frac{\xi}{l}\right)^{\frac{1}{2}}\right\rangle^{-1}}{l^{2}\left(1+\left|t-\frac{\xi}{l}\right|^{2}\right)} & \lesssim \sqrt{\frac{|l|}{l^{2}\left(1+\left|t-\frac{\xi}{l}\right|^{2}\right)}} \sqrt{\frac{|l|}{l^{2}\left(1+\left|t-\frac{\eta}{l}\right|^{2}\right)}}\langle\xi-\eta\rangle\left\langle v^{\frac{1}{2}}\left(\frac{\xi}{l}\right)^{\frac{1}{2}}\right\rangle^{-1} \\
& \lesssim \sqrt{\frac{\xi}{l^{2}\left(1+\left|t-\frac{\xi}{l}\right|^{2}\right)}} \sqrt{\frac{\eta}{l^{2}\left(1+\left|t-\frac{\eta}{l}\right|^{2}\right)}}\langle\xi-\eta\rangle\left\langle v^{\frac{1}{3}}\left(\frac{\xi}{l}\right)\right\rangle^{-(1+\beta)} \\
& \lesssim \sqrt{\frac{\partial_{t} w(t, \eta)}{w(t, \eta)}} \sqrt{\frac{\partial_{t} w(t, \xi)}{w(t, \xi)}}\langle\xi-\eta\rangle v^{-\frac{1}{3}}
\end{aligned}
$$

which gives

$$
\begin{aligned}
\left|R_{N,=}^{\mathrm{R}, \mathrm{R}}\right| & \lesssim\left\|\sqrt{\frac{\partial_{t} w}{w}} A^{\sigma} f_{\sim N}\right\|\left\|_{L^{2}}\right\| \sqrt{\frac{\partial_{t} w}{w}} \chi_{R} A^{\sigma} \Delta_{L} \Delta_{t}^{-1} f_{N}\left\|_{L^{2}}\right\| v^{-\frac{1}{3}}\left(v^{\frac{1}{2}} t^{\frac{1}{2}} \partial_{v}\right) f_{0} \|_{H^{3}} \\
& \lesssim \varepsilon\left\|\sqrt{\frac{\partial_{t} w}{w}} A^{\sigma} f_{\sim N}\right\|_{L^{2}}\left\|\sqrt{\frac{\partial_{t} w}{w}} \chi_{R} A^{\sigma} \Delta_{L} \Delta_{t}^{-1} f_{N}\right\|_{L^{2}} \cdot
\end{aligned}
$$

8.1.6. Treatment of $\boldsymbol{R}_{N, \neq, *}$. In this case we get $(l, k, \xi, \eta) \notin D_{1}$ which means that at least one of the two inequalities $|l| \geq|\xi|,|l-k, \xi-\eta| \geq \frac{1}{1000}|l, \xi|$ holds. Thus we get

$$
\frac{A_{k}^{\sigma}(\eta)|l, \xi|}{l^{2}+|l t-\xi|^{2}} \lesssim A_{l}^{\sigma}(\xi)\langle l-k, \xi-\eta\rangle,
$$

which implies

$$
\begin{aligned}
\left|R_{N, \neq, *}\right| & \lesssim\left\|A^{\sigma} f_{\sim N}\right\|_{2}\left\|A^{\sigma} \Delta_{L} \Delta_{t}^{-1} f_{N}\right\|_{2}\left\|f_{\neq}\right\|_{H^{4}} \\
& \lesssim \frac{\varepsilon v^{\frac{1}{3}}}{\left\langle v t^{3}\right\rangle}\left\|A^{\sigma} f_{\sim N}\right\|_{2}\left\|A^{\sigma} \Delta_{L} \Delta_{t}^{-1} f_{N}\right\|_{2} .
\end{aligned}
$$

In the next four subsections, we restrict $(l, k, \xi, \eta) \in D_{1}$, which gives $|\eta| \geq \frac{1}{20}|k|$ and $|\xi| \geq \frac{1}{20}|l| ;$ thus

$$
w_{k}(t, \eta)=w(t, \eta), \quad w_{l}(t, \xi)=w(t, \xi)
$$


8.1.7. Treatment of $\boldsymbol{R}_{\boldsymbol{N}}^{\mathrm{NR}, \mathrm{NR}}$. Since we restrict the integrand to $D_{1}$, it holds that

$$
|| l, \xi|-| k, \eta|| \leq|k-l, \eta-\xi| \leq \frac{1}{1000}|l, \xi| .
$$

It follows from the fact that

$$
(\eta l-\xi k)=(\eta-\xi) l+(l-k) \xi
$$

and Lemma 3.3 that

$$
\begin{aligned}
\left|R_{N}^{\mathrm{NR}, \mathrm{NR}}\right| & \lesssim \sum_{k, l \neq 0, k \neq l} \int_{\eta, \xi} 1_{t \notin I_{k, \eta}, t \notin I_{l, \xi}}\left|A^{\sigma} \overline{\hat{f}}_{k}(\eta)\right| \\
& \times\left|\frac{A_{l}^{\sigma}(\xi)|l, \xi|}{l^{2}+(\xi-l t)^{2}} \widehat{\Delta_{L} \Delta_{t}^{-1} f_{l}}(\xi)_{N} \widehat{\nabla f f}_{k-l}(\eta-\xi)_{<N / 8}\right| d \eta d \xi \\
& \lesssim \sum_{k, l \neq 0, k \neq l} \int_{\eta, \xi} 1_{t \notin I_{k, \eta}, t \notin I_{l, \xi}} A^{\sigma}\left|\overline{\hat{f}}_{k}(\eta)\right| \frac{A_{l}^{\sigma}(\xi)|l, \xi|}{l^{2}\left(1+\frac{\xi^{2}}{l^{4}}\right)} \\
& \times\left|{\widehat{\Delta} L \Delta_{t}^{-1} f_{l}}_{l \xi}(\xi)_{N} \widehat{\nabla f}_{k-l}(\eta-\xi)_{<N / 8}\right| d \eta d \xi .
\end{aligned}
$$

Therefore we have

$$
\begin{aligned}
\left|R_{N}^{\mathrm{NR}, \mathrm{NR}}\right| & \lesssim\left\|A^{\sigma} f_{\sim N}\right\|_{2}\left\|A^{\sigma} \Delta_{L} \Delta_{t}^{-1} f_{N}\right\|_{2}\left\|f_{\neq}\right\|_{H^{3}} \\
& \lesssim \frac{\varepsilon v^{\frac{1}{3}}}{\left\langle\nu t^{3}\right\rangle}\left\|A^{\sigma} f_{\sim N}\right\|_{2}\left\|A^{\sigma} \Delta_{L} \Delta_{t}^{-1} f_{N}\right\|_{2} .
\end{aligned}
$$

8.1.8. Treatment of $\boldsymbol{R}_{\boldsymbol{N}}^{\mathbf{N R}, \mathbf{R}}$. By (8.13) we have $\langle k, \eta\rangle^{\sigma} \approx\langle l, \xi\rangle^{\sigma}$ and $A_{l}(\xi) \approx A_{k}(\eta)$, which gives

$$
\begin{aligned}
\left|R_{N}^{\mathrm{NR}, \mathrm{R}}\right| \lesssim & \sum_{k, l \neq 0, k \neq l} \int_{|\eta-\xi| \leq \frac{|\eta|}{100}} \chi^{\mathrm{NR}, \mathrm{R}}\left|A^{\sigma} \overline{\hat{f}}_{k}(\eta)\right| \frac{A_{l}^{\sigma}(\xi)|l, \xi|}{l^{2}+(\xi-l t)^{2}} \\
& \times\left|\widehat{\Delta_{L} \Delta_{t}^{-1} f_{l}}(\xi)_{N} \widehat{\nabla f}_{k-l}(\eta-\xi)_{<N / 8}\right| d \eta d \xi \\
& +\sum_{k, l \neq 0, k \neq l} \int_{|\eta-\xi|>\frac{|\eta|}{100}} \chi^{\mathrm{NR}, \mathrm{R}}\left|A^{\sigma} \overline{\hat{f}}_{k}(\eta)\right| \frac{A_{l}^{\sigma}(\xi)|l, \xi|}{l^{2}+(\xi-l t)^{2}} \\
= & \times\left|\widehat{\Delta_{L} \Delta_{t}^{-1} f_{l}}(\xi)_{N} \widehat{\nabla f}_{k-l}(\eta-\xi)_{<N / 8}\right| d \eta d \xi \\
&
\end{aligned}
$$

Let us first deal with $R_{N, 1}^{\mathrm{NR}, \mathrm{R}}$, so that $|\eta-\xi| \leq \frac{|\eta|}{100}$.

If $t \leq t(\eta) \approx \sqrt{|\eta|}$, then $w_{k}(\eta)=1$ and by the fact that $t \geq t(\xi) \approx \sqrt{|\eta|}$, we get that $t \approx \sqrt{|\xi|},|l| \approx \sqrt{|\xi|}$ and then

$$
\begin{aligned}
\left|R_{N, 1}^{\mathrm{NR}, \mathrm{R}}\right| & \lesssim \sum_{k, l \neq 0, k \neq l} \int_{\eta, \xi} 1_{t \notin I_{k, \eta}, t \in I_{l, \xi}}\left|A^{\sigma} \overline{\hat{f}}_{k}(\eta)\right| A^{\sigma} \\
& \times \mid \widehat{\Delta_{L} \Delta_{t}^{-1} f_{l}}(\xi)_{N} \widehat{\nabla f} \\
& \lesssim\left\|A^{\sigma} f_{\sim N}\right\|_{2}\left\|A^{\sigma} \Delta_{L} \Delta_{t}^{-1} f_{N}\right\|_{2}\left\|f_{\neq}\right\|_{H^{3}} .
\end{aligned}
$$


If $t \geq 2|\eta|$, and for $|\eta-\xi| \leq \frac{1}{100}|\eta|$ we have $|l| t-|\xi| \geq 2|\eta|-|\xi| \geq|\eta|-|| \eta|-| \xi|| \gtrsim$ $|\xi|$, this implies

$$
\left|R_{N, 1}^{\mathrm{NR}, \mathrm{R}}\right| \lesssim\left\|A^{\sigma} f_{\sim N}\right\|_{L^{2}}\left\|A^{\sigma} \Delta_{L} \Delta_{t}^{-1} f_{N}\right\|_{H^{\sigma}}\left\|f_{\neq}\right\|_{H^{3}} .
$$

If $t \in I_{t}(\eta)$, there exists $k^{\prime} \in[1, E(\sqrt{|\eta|})]$ such that $t \in I_{k^{\prime}, \eta} \cap I_{l, \xi}$. Then by Lemma 3.2, we need to consider the following three cases:

(a) $k^{\prime}=l$. In this case, by using the fact that

$$
\begin{aligned}
\frac{|\xi|}{l^{2}+(\xi-l t)^{2}} & \lesssim \sqrt{\frac{|\xi|}{l^{2}+(\xi-l t)^{2}}} \sqrt{\frac{|\eta|}{l^{2}+(\eta-l t)^{2}}}\langle\xi-\eta\rangle \\
& \lesssim\left\langle v^{\frac{1}{3}} t\right\rangle^{1+\beta} v^{-\frac{1}{3}}\langle\xi-\eta\rangle \sqrt{\frac{\partial_{t} w(t, \xi)}{w(t, \xi)}} \sqrt{\frac{\partial_{t} w(t, \eta)}{w(t, \eta)}}
\end{aligned}
$$

and $|l| \lesssim \sqrt{|\xi|} \lesssim|\xi|$, we get

$$
\begin{aligned}
& \left|R_{N, 1}^{\mathrm{NR}, \mathrm{R}}\right| \lesssim \sum_{k, l \neq 0, k \neq l} \int_{|\eta-\xi| \leq \frac{|\eta|}{100}} 1_{t \in I_{k^{\prime}, \eta} \cap I_{l, \xi} \mid} A^{\sigma} \overline{\hat{f}}_{k}(\eta) \mid \frac{A_{l}^{\sigma}(t, \xi)|\xi|}{l^{2}+(\xi-l t)^{2}}
\end{aligned}
$$

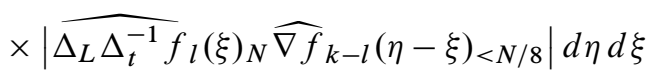

$$
\begin{aligned}
& \lesssim \sum_{k, l \neq 0, k \neq l} \int_{|\eta-\xi| \leq \frac{1}{100}|\eta|} 1_{t \in I_{l, \eta} \cap I_{l, \xi}}\left|\sqrt{\frac{\partial_{t} w(\eta)}{w(\eta)}} A^{\sigma} \overline{\hat{f}}_{k}(\eta)\right| \\
& \times \mid \sqrt{\frac{\partial_{t} w(\eta)}{w(\eta)}} A^{\sigma} \widehat{\Delta_{L} \Delta_{t}^{-1}} f_{l}(\xi)_{N}\left(1+v^{\frac{1}{3}} t\right)^{1+\beta} v^{-\frac{1}{3}} \\
& \times{\widehat{\left\langle\nabla \partial_{v}\right\rangle}}_{k-l}(\eta-\xi)_{<N / 8} \mid d \eta d \xi \\
& \lesssim\left\|\sqrt{\frac{\partial_{t} w(\eta)}{w(\eta)}} A^{\sigma} f_{\sim N}\right\|_{2}\left\|\sqrt{\frac{\partial_{t} w(\xi)}{w(\xi)}} \chi_{R} A^{\sigma} \Delta_{L} \Delta_{t}^{-1} f_{N}\right\|_{2}\left\|\left\langle v^{\frac{1}{3}} t\right\rangle^{1+\beta} v^{-\frac{1}{3}} f_{\neq}\right\|_{H^{4}} .
\end{aligned}
$$

(b) $\left|t-\frac{\eta}{k^{\prime}}\right| \gtrsim \frac{\eta}{k^{\prime 2}}$ and $\left|t-\frac{\xi}{l}\right| \gtrsim \frac{\xi}{l^{2}}$. In this case, by using the fact that

$$
\frac{|l, \xi|}{l^{2}+(\xi-l t)^{2}} \lesssim \frac{|\xi| / l^{2}}{1+(\xi / l-t)^{2}} \lesssim \frac{|\xi| / l^{2}}{1+\frac{\xi^{2}}{l^{4}}} \lesssim 1
$$

we obtain

$$
\left|R_{N, 1}^{\mathrm{NR}, \mathrm{R}}\right| \lesssim\left\|A^{\sigma} f_{\sim N}\right\|_{L^{2}}\left\|A^{\sigma} \Delta_{L} \Delta_{t}^{-1} P_{\neq} f_{N}\right\|_{L^{2}}\left\|f_{\neq}\right\|_{H^{3}} .
$$

(c) $|\eta-\xi| \gtrsim \frac{|\eta|}{|l|} \approx \frac{|\xi|}{|l|}$. In this case we get

$$
\frac{|\xi|}{l^{2}+(\xi-l t)^{2}} \lesssim \frac{|\xi|}{l^{2}} \lesssim|\eta-\xi|
$$


which gives

$$
R_{N, 1}^{\mathrm{NR}, \mathrm{R}} \lesssim\left\|A^{\sigma} f_{\sim N}\right\|_{L^{2}}\left\|A^{\sigma} \Delta_{L} \Delta_{t}^{-1} P_{\neq} f_{N}\right\|_{L^{2}}\left\|f_{\neq}\right\|_{H^{4}} .
$$

Next we deal with $R_{N, 2}^{\mathrm{NR}, \mathrm{R}}$, for which we will use the fact that

$$
\frac{|l, \xi|}{l^{2}+(\xi-l t)^{2}} \lesssim 1+\frac{|\xi|}{l^{2}} \lesssim\langle\xi-\eta\rangle .
$$

Thus we get

$$
\begin{aligned}
R_{N, 2}^{\mathrm{NR}, \mathrm{R}} & \lesssim \sum_{k, l \neq 0, k \neq l} \int_{|\eta-\xi|>\frac{|\eta|}{100}} \chi^{\mathrm{NR}, \mathrm{R}}\left|A^{\sigma} \overline{\hat{f}}_{k}(\eta)\right| \frac{A_{l}^{\sigma}(\xi)|l, \xi|}{l^{2}+(\xi-l t)^{2}} \\
& \times\left|\widehat{\Delta_{L} \Delta_{t}^{-1}} f_{l}(\xi)_{N} \widehat{\nabla f}_{k-l}(\eta-\xi)_{<N / 8}\right| d \eta d \xi \\
& \lesssim\left\|A^{\sigma} f_{\sim N}\right\|_{2}\left\|A^{\sigma} \Delta_{L} \Delta_{t}^{-1} P_{\neq} f_{N}\right\|_{L^{2}}\left\|f_{\neq}\right\|_{H^{4}} .
\end{aligned}
$$

Therefore we conclude that

$$
\begin{aligned}
\left|R_{N}^{\mathrm{NR}, \mathrm{R}}\right| \lesssim & \left\|A^{\sigma} f_{\sim N}\right\|_{L^{2}}\left\|A^{\sigma} \Delta_{L} \Delta_{t}^{-1} f_{N}\right\|_{L^{2}}\left\|f_{\neq}\right\|_{H^{4}} \\
& +\left\|\sqrt{\frac{\partial_{t} w}{w}} A^{\sigma} f_{\sim N}\right\|\left\|\sqrt{\frac{\partial_{t} w}{w}} \chi_{R} A^{\sigma} \Delta_{L} \Delta_{t}^{-1} f_{N}\right\|_{2}\left\|\left\langle v^{\frac{1}{3}} t\right\rangle^{1+\beta} v^{-\frac{1}{3}} f_{\neq}\right\|_{H^{4}} \\
\lesssim & \frac{\varepsilon v^{\frac{1}{3}}}{\left\langle v t^{3}\right\rangle}\left\|A^{\sigma} f_{\sim N}\right\|_{L^{2}}\left\|A^{\sigma} \Delta_{L} \Delta_{t}^{-1} f_{N}\right\|_{L^{2}} \\
& +\varepsilon\left\|\sqrt{\frac{\partial_{t} w}{w}} A^{\sigma} f_{\sim N}\right\|_{2}\left\|\sqrt{\frac{\partial_{t} w}{w}} \chi_{R} A^{\sigma} \Delta_{L} \Delta_{t}^{-1} f_{N}\right\|_{2}
\end{aligned}
$$

8.1.9. Treatment of $R_{N}^{R, N R}$. In this case we have

$$
\frac{|l, \xi|}{l^{2}+(\xi-l t)^{2}} \lesssim \begin{cases}\frac{|l|}{l^{2}\left(1+\frac{|\xi|^{2}}{l^{4}}\right)} \lesssim 1 & \text { if }|l| \leq E(\sqrt{\xi}), \\ \frac{1}{\sqrt{\xi}} \lesssim 1 & \text { if }|l| \geq E(\sqrt{\xi})+1\end{cases}
$$

which implies

$$
\begin{aligned}
R_{N}^{\mathrm{R}, \mathrm{NR}} & \lesssim \sum_{k, l \neq 0, k \neq l} \int_{\eta, \xi} \chi^{\mathrm{R}, \mathrm{NR}}\left|A^{\sigma} \overline{\hat{f}}_{k}(\eta)\right| \frac{A_{l}^{\sigma}(\xi)|l, \xi|}{l^{2}+(\xi-l t)^{2}} \\
& \times\left|\widehat{\Delta_{L} \Delta_{t}^{-1} f_{l}}(\xi)_{N} \widehat{\nabla f} \widehat{\nabla}_{k-l}(\eta-\xi)_{<N / 8}\right| d \eta d \xi \\
& \lesssim\left\|A^{\sigma} f_{\sim N}\right\|_{2}\left\|A^{\sigma} \Delta_{L} \Delta_{t}^{-1} P_{\neq} f_{N}\right\|_{L^{2}}\left\|f_{\neq}\right\|_{H^{3}} \\
& \lesssim \frac{\varepsilon v^{\frac{1}{3}}}{\left\langle v t^{3}\right\rangle}\left\|A^{\sigma} f_{\sim N}\right\|_{2}\left\|A^{\sigma} \Delta_{L} \Delta_{t}^{-1} P_{\neq} f_{N}\right\|_{L^{2}} .
\end{aligned}
$$


8.1.10. Treatment of $\boldsymbol{R}_{\boldsymbol{N}}^{\mathbf{R}, \mathbf{R}}$. In this case $t \in I_{k, \eta} \cap I_{l, \xi}$ with $k \neq l$. By Lemma 3.2, we only need to deal with the following two cases:

(b) $\left|t-\frac{\eta}{k}\right| \gtrsim \frac{\eta}{k^{2}}$ and $\left|t-\frac{\xi}{l}\right| \gtrsim \frac{\xi}{l^{2}}$. In this case, by the fact that $A_{l}^{\sigma}(\xi) \approx A_{k}^{\sigma}(\eta)$ and

$$
\frac{|l, \xi|}{l^{2}+(\xi-l t)^{2}} \lesssim 1+\frac{|\xi|}{l^{2}\left(1+\frac{\xi^{2}}{l^{4}}\right)} \lesssim 1
$$

we get

$$
\begin{aligned}
\left|R_{N}^{\mathrm{R}, \mathrm{R}}\right| & \lesssim \sum_{k, l \neq 0, k \neq l} \int_{\eta, \xi} \chi^{\mathrm{R}, \mathrm{R}} A^{\sigma}\left|\overline{\hat{f}}_{k}(\eta)\right| \frac{A_{l}^{\sigma}(\xi)|l, \xi|}{l^{2}+(\xi-l t)^{2}} \\
& \times\left|{\widehat{\Delta_{L} \Delta_{t}^{-1}}}_{l}(\xi)_{N} \widehat{\nabla f}_{k-l}(\eta-\xi)_{<N / 8}\right| d \eta d \xi \\
& \lesssim\left\|A^{\sigma} f_{\sim N}\right\|_{L^{2}}\left\|A^{\sigma} \Delta_{L} \Delta_{t}^{-1} f_{N}\right\|_{L^{2}}\left\|f_{\neq}\right\|_{H^{3}} .
\end{aligned}
$$

(c) $|\eta-\xi| \gtrsim \frac{|\eta|}{|l|} \approx \frac{|\xi|}{|l|}$. In this case, by using the fact that $A_{l}^{\sigma}(\xi) \approx A_{k}^{\sigma}(\eta)$ and

$$
\frac{|l, \xi|}{l^{2}+(\xi-l t)^{2}} \lesssim 1+\frac{|\xi|}{l^{2}} \lesssim|\eta-\xi|,
$$

we get

$$
\begin{aligned}
\left|R_{N}^{\mathrm{R}, \mathrm{R}}\right| & \lesssim \sum_{k, l \neq 0, k \neq l} \int_{\eta, \xi} \chi^{\mathrm{R}, \mathrm{R}} A^{\sigma}\left|\overline{\hat{f}}_{k}(\eta)\right| \frac{A_{l}(\xi)|l, \xi|}{l^{2}+(\xi-l t)^{2}} \\
& \times\left|\widehat{\Delta_{L} \Delta_{t}^{-1} f_{l}}(\xi)_{N} \widehat{\nabla f}_{k-l}(\eta-\xi)_{<N / 8}\right| d \eta d \xi \\
& \lesssim\left\|A^{\sigma} f_{\sim N}\right\|_{2}\left\|A^{\sigma} \Delta_{L} \Delta_{t}^{-1} f_{N}\right\|_{2}\left\|f_{\neq}\right\|_{H^{4}} .
\end{aligned}
$$

Therefore we conclude that

$$
\begin{aligned}
\left|R_{N}^{\mathrm{R}, \mathrm{R}}\right| & \lesssim\left\|A^{\sigma} f_{\sim N}\right\|_{2}\left\|A^{\sigma} \Delta_{L} \Delta_{t}^{-1} P_{\neq} f_{N}\right\|_{2}\left\|f_{\neq}\right\|_{H^{4}} \\
& \lesssim \frac{\varepsilon v^{\frac{1}{3}}}{\left\langle v t^{3}\right\rangle}\left\|A^{\sigma} f_{\sim N}\right\|_{2}\left\|A^{\sigma} \Delta_{L} \Delta_{t}^{-1} P_{\neq} f_{N}\right\|_{2} .
\end{aligned}
$$

Combining (8.1), (8.7), (8.8), (8.9), (8.10), (8.11), (8.14), (8.16), (8.17) and (8.18), we deduce that

$$
\begin{aligned}
\left|R_{N}^{1}\right| \lesssim & \left(\frac{\varepsilon v^{\frac{1}{3}}}{\left\langle v^{\frac{1}{2}} t^{\frac{3}{2}}\right\rangle}+\frac{\varepsilon v^{\frac{1}{3}}}{\left\langle t^{2}\right\rangle}+\frac{\varepsilon v^{\frac{1}{3}}}{\left\langle v t^{3}\right\rangle}\right)\left\|A^{\sigma} f_{\sim N}\right\|_{L^{2}}\left\|A^{\sigma} \Delta_{L} \Delta_{t}^{-1} P_{\neq} f_{N}\right\|_{L^{2}} \\
& +\varepsilon\left\|\sqrt{\frac{\partial_{t} w}{w}} A^{\sigma} f_{\sim N}\right\|_{2}\left\|\sqrt{\frac{\partial_{t} w}{w}} \chi_{R} A^{\sigma} \Delta_{L} \Delta_{t}^{-1} P_{\neq} f_{N}\right\|_{2}
\end{aligned}
$$




\subsection{Treatment of $R_{N}^{2}$}

We recall that

$$
\begin{aligned}
R_{N}^{2}= & \sum_{k \neq 0} \int_{\eta, \xi} A^{\sigma} \overline{\hat{f}}_{k}(\eta) A_{k}^{\sigma}(\eta) \hat{g}(\xi)_{N}{\widehat{\partial f_{v}}}_{k}(\eta-\xi)_{<N / 8} d \eta d \xi \\
& +\int_{\eta, \xi} A^{\sigma} \overline{\hat{f}}_{0}(\eta) A_{0}^{\sigma}(\eta) \hat{g}(\xi)_{N}{\widehat{\partial f_{v} f}}_{0}(\eta-\xi)_{<N / 8} d \eta d \xi \\
= & R_{N, \neq}^{2}+R_{N, 0}^{2} .
\end{aligned}
$$

By the fact that $|k, \eta-\xi| \leq \frac{3}{16} N \leq \frac{3}{8}|\xi| \approx|k, \eta|$, we have

$$
\left|R_{N, \neq}^{2}\right| \lesssim\left\|A^{\sigma}\left(f_{\neq}\right)_{\sim N}\right\|_{2}\left\|\left\langle\partial_{v}\right\rangle^{\sigma} g_{N}\right\|_{2}\left\|f_{\neq}\right\|_{H^{3}}
$$

and

$$
\left|R_{N, 0}^{2}\right| \lesssim\left\|A^{\sigma}\left(f_{0}\right)_{\sim}\right\|_{2}\left\|\left\langle\partial_{v}\right\rangle^{\sigma} g_{N}\right\|_{2}\left\|f_{0}\right\|_{H^{3}} .
$$

Thus we obtain

$$
\left|R_{N}^{2}\right| \lesssim\left\|A^{\sigma} f_{\sim N}\right\|_{2}\left\|g_{N}\right\|_{H^{\sigma}}\|f\|_{H^{3}}
$$

\subsection{Treatment of $R_{N}^{3}$}

The term $R_{N}^{3}$ is easy to deal with, because the derivatives land on the low frequency. We then get

$$
\begin{aligned}
\left|R_{N}^{3}\right| & \leq\left|\sum_{k, l} \int_{\eta, \xi} A^{\sigma} \overline{\hat{f}}_{k}(\eta) A_{k-l}^{\sigma}(\eta-\xi) \hat{u}_{l}(\xi)_{N} \widehat{\nabla f}_{k-l}(\eta-\xi)_{<N / 8} d \eta d \xi\right| \\
& \leq \sum_{k, l} \int_{\eta, \xi} A^{\sigma}\left|\overline{\hat{f}}_{k}(\eta)\right||l, \xi|\left|\hat{u}_{l}(\xi)_{N}\right| A_{k-l}^{\sigma}(\eta-\xi)\left|\hat{f}_{k-l}(\eta-\xi)_{<N / 8}\right| d \eta d \xi
\end{aligned}
$$

which gives

$$
\left|R_{N}^{3}\right| \lesssim\left\|A^{\sigma} f_{\sim N}\right\|_{2}\left\|u_{N}\right\|_{H^{3}}\|f\|_{H^{\sigma}}
$$

\subsection{Corrections}

In this section we treat $R_{N}^{\varepsilon, 1}$, which is higher order in $v^{\frac{1}{3}}$ than $R_{N}^{1}$. We expand $\left(1-v^{\prime}\right) \phi_{l}$ with a paraproduct only in $v$ :

$$
\begin{aligned}
& R_{N}^{\varepsilon, 1}=-\frac{1}{2 \pi} \sum_{M \geq 8} \sum_{k, l \neq 0} \int_{\eta, \xi, \xi^{\prime}} A^{\sigma} \overline{\hat{f}}_{k}(\eta) A_{k}^{\sigma}(\eta)\left((\xi-\eta) l-\xi^{\prime}(k-l)\right) \chi_{N}(l, \xi) \\
& \left.\quad \times\left[\widehat{\left(1-v^{\prime}\right.}\right)\left(\xi^{\prime}-\xi\right)\right]_{<M / 8} \widehat{\phi}_{l}\left(\xi^{\prime}\right)_{M} \hat{f_{k-l}(\eta-\xi)_{<N / 8} d \eta d \xi d \xi^{\prime}} \\
& -\frac{1}{2 \pi} \sum_{M \geq 8} \sum_{k, l \neq 0} \int_{\eta, \xi, \xi^{\prime}} A^{\sigma} \overline{\hat{f}}_{k}(\eta) A_{k}^{\sigma}(\eta)\left((\xi-\eta) l-\xi^{\prime}(k-l)\right) \chi_{N}(l, \xi) \\
& \left.\times\left[\widehat{\left(1-v^{\prime}\right.}\right)\left(\xi^{\prime}-\xi\right)\right]_{M} \widehat{\phi}_{l}\left(\xi^{\prime}\right)_{<M / 8} \hat{f}_{k-l}(\eta-\xi)_{<N / 8} d \eta d \xi d \xi^{\prime}
\end{aligned}
$$




$$
\begin{aligned}
& -\frac{1}{2 \pi} \sum_{M \in \mathbb{D}} \sum_{\frac{1}{8} M \leq M^{\prime} \leq M} \sum_{k, l \neq 0} \int_{\eta, \xi, \xi^{\prime}} A^{\sigma} \overline{\hat{f}}_{k}(\eta) A_{k}^{\sigma}(\eta)\left((\xi-\eta) l-\xi^{\prime}(k-l)\right) \\
& \times \chi_{N}(l, \xi)\left[\widehat{\left(1-v^{\prime}\right)}\left(\xi^{\prime}-\xi\right)\right]_{M^{\prime}} \widehat{\phi}_{l}\left(\xi^{\prime}\right)_{M} \hat{f}_{k-l}(\eta-\xi)_{<N / 8} d \eta d \xi d \xi^{\prime} \\
& =R_{N, L H}^{\varepsilon, 1}+R_{N, H L}^{\varepsilon, 1}+R_{N, H H}^{\varepsilon, 1} .
\end{aligned}
$$

We recall that $\chi_{N}$ denotes the Littlewood-Paley cutoff to the $N$ th dyadic shell in $\mathbb{Z} \times \mathbf{R}$; see Section A.1.

We begin with $R_{N, L H}^{\varepsilon, 1}$. On the support of the integrand,

$$
\begin{aligned}
& || k, \eta|-| l, \xi|| \leq|k-l, \eta-\xi| \leq \frac{3}{8}|l, \xi|, \\
& || l, \xi^{\prime}|-| l, \xi|| \leq\left|\xi-\xi^{\prime}\right| \leq \frac{3}{8}\left|l, \xi^{\prime}\right| .
\end{aligned}
$$

Thus $A_{k}^{\sigma}(\eta) \approx A_{l}^{\sigma}\left(\xi^{\prime}\right)$ and

$$
\begin{aligned}
\left|R_{N, L H}^{\varepsilon, 1}\right| \lesssim \sum_{M \geq 8} & \sum_{k, l \neq 0} \int_{\eta, \xi, \xi^{\prime}}\left|A^{\sigma} \overline{\hat{f}}_{k}(\eta)\right|\left|(\xi-\eta) l-\xi^{\prime}(k-l)\right| \chi_{N}(l, \xi) \\
& \times \mid\left[\left(\overline{\left(-v^{\prime}\right)}\left(\xi^{\prime}-\xi\right)\right]_{<M / 8} A_{l}^{\sigma}\left(\xi^{\prime}\right) \hat{\phi}_{l}\left(\xi^{\prime}\right)_{M} \hat{f}_{k-l}(\eta-\xi)_{<N / 8} \mid d \eta d \xi d \xi^{\prime} .\right.
\end{aligned}
$$

From here we may proceed analogously to the treatment of $R_{N}^{1}$ with $\left(l, \xi^{\prime}\right)$ playing the role of $(l, \xi)$. We omit the details and simply conclude the result is

$$
\begin{aligned}
\left|R_{N, L H}^{\varepsilon, 1}\right| \lesssim\|h\|_{H^{3}}[ & \left(\frac{\varepsilon \nu^{\frac{1}{3}}}{\left\langle t^{\frac{3}{2}} \nu^{\frac{1}{2}}\right\rangle}+\frac{\varepsilon v^{\frac{1}{3}}}{\left\langle t^{2}\right\rangle}+\frac{\varepsilon \nu^{\frac{1}{3}}}{\left\langle t^{3} v\right\rangle}\right)\left\|A^{\sigma} f_{\sim N}\right\|_{L^{2}}\left\|A^{\sigma} \Delta_{L} \Delta_{t}^{-1} P_{\neq} f_{N}\right\|_{L^{2}} \\
& \left.+\varepsilon\left\|\sqrt{\frac{\partial_{t} w}{w}} A^{\sigma} f_{\sim N}\right\|_{2}\left\|\sqrt{\frac{\partial_{t} w}{w}} A^{\sigma} \chi_{R} \Delta_{L} \Delta_{t}^{-1} P_{\neq} f_{N}\right\|_{2}\right] .
\end{aligned}
$$

Turn now to $R_{N, H L}^{\varepsilon, 1}$. On the support of the integrand, it holds that

$$
\langle k, \eta\rangle^{\sigma} \approx\langle l, \xi\rangle^{\sigma} \approx\left\langle l, \xi^{\prime}-\xi\right\rangle^{\sigma} .
$$

Thus we get

$$
\begin{aligned}
& \left|R_{N, H L}^{\varepsilon, 1}\right| \lesssim \sum_{M \geq 8} \sum_{k, l \neq 0} \int_{\eta, \xi, \xi^{\prime}} \chi_{|l| \geq \mid \frac{\xi \xi}{16}}\left|A^{\sigma} \overline{\hat{f}}_{k}(\eta)\right| \chi_{N}(l, \xi) \mid\left[\widehat{\left(1-v^{\prime}\right)}\left(\xi^{\prime}-\xi\right)\right]_{M} \\
& \times\langle l\rangle^{\sigma}\left|l, \xi^{\prime}\right| \widehat{\phi}_{l}\left(\xi^{\prime}\right)_{<M / 8} \widehat{\nabla f}_{k-l}(\eta-\xi)_{<N / 8} \mid d \eta d \xi d \xi^{\prime} \\
& \left.+\sum_{M \geq 8} \sum_{k, l \neq 0} \int_{\eta, \xi, \xi^{\prime}} \chi_{|l|<\left|\frac{\xi}{16}\right|}\left|A^{\sigma} \overline{\hat{f}}_{k}(\eta)\right| \chi_{N}(l, \xi)\left\langle\xi^{\prime}-\xi\right\rangle^{\sigma} \mid\left[\widehat{\left(1-v^{\prime}\right.}\right)\left(\xi^{\prime}-\xi\right)\right]_{M} \\
& \times\left|l, \xi^{\prime}\right| \widehat{\phi}_{l}\left(\xi^{\prime}\right)_{<M / 8} \widehat{\nabla f}_{k-l}(\eta-\xi)_{<N / 8} \mid d \eta d \xi d \xi^{\prime} \\
& =R_{N, H L}^{\varepsilon, 1, z}+R_{N, H L}^{\varepsilon, 1, v} \text {. }
\end{aligned}
$$


First, consider $R_{N, H L}^{\varepsilon, 1, z}$, where on the support of the integrand, $16|l| \geq|\xi|$ :

$$
\begin{gathered}
|k, \eta|-|l, \xi||\leq| k-l, \xi-\eta\left|\leq \frac{3}{16}\right| l, \xi \mid, \\
|| l, \xi|-| l, \xi^{\prime}|| \leq\left|\xi-\xi^{\prime}\right| \leq \frac{38|\xi|}{32} \lesssim|l| .
\end{gathered}
$$

Thus we divide $R_{N, H L}^{\varepsilon, 1, z}$ into two parts,

$$
\begin{aligned}
& \left.R_{N, H L}^{\varepsilon, 1, z} \lesssim \sum_{M \geq 8} \sum_{k, l \neq 0} \int_{\eta, \xi, \xi^{\prime}} \chi|l| \geq 16|\xi|\left|A^{\sigma} \overline{\hat{f}}_{k}(\eta)\right| \chi_{N}(l, \xi) \mid\left[\widehat{\left(1-v^{\prime}\right.}\right)\left(\xi^{\prime}-\xi\right)\right]_{M} \\
& \times\left[\langle l\rangle^{\sigma+1} \widehat{\phi}_{l}\left(\xi^{\prime}\right)_{<M / 8}\right]_{\sim N} \widehat{\nabla f}_{k-l}(\eta-\xi)_{<N / 8} \mid d \eta d \xi d \xi^{\prime}
\end{aligned}
$$

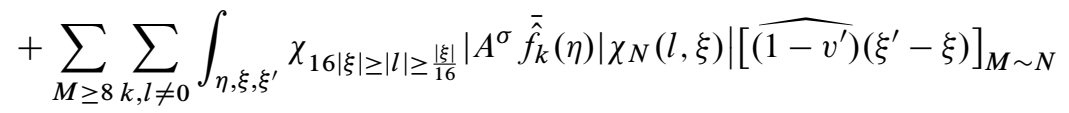

$$
\begin{aligned}
& \times\langle l\rangle^{\sigma}\left|l, \xi^{\prime}\right| \widehat{\phi}_{l}\left(\xi^{\prime}\right)_{<M / 8} \widehat{\nabla f}_{k-l}(\eta-\xi)_{<N / 8} \mid d \eta d \xi d \xi^{\prime} \\
& =R_{N, H L, 1}^{\varepsilon, 1, z}+R_{N, H L, 2}^{\varepsilon, 1, z}
\end{aligned}
$$

To make it summable in $M$ we need more "derivatives" at higher (in $M$ ) frequency; luckily, all of the "derivatives" land on the lower (in $M$ ) frequency. gives

If $|l| \geq 16|\xi|$, then in fact $\frac{38|\xi|}{32} \leq \frac{|l|}{4}$, and therefore ||$k, \eta|-| l, \xi^{\prime}|| \leq \frac{|l|}{2} \leq \frac{\left|l, \xi^{\prime}\right|}{2}$, which

$$
|k, \eta| \approx\left|l, \xi^{\prime}\right| \approx|l| \approx N .
$$

For $|l| \geq 16|\xi| \geq 16\left|\xi^{\prime}\right|$, we get

$$
\frac{|l|}{l^{2}\left(1+\left(\frac{\xi^{\prime}}{l}-t\right)^{2}\right)} \lesssim \frac{|l|^{-1}\left\langle\frac{\left|\xi^{\prime}\right|}{|l|}\right\rangle^{2}}{\left\langle\frac{\xi^{\prime} \mid}{|l|}\right\rangle^{2}\left(1+\left(\frac{\xi^{\prime}}{l}-t\right)^{2}\right)} \lesssim \frac{|l|^{-1}}{\langle t\rangle^{2}} .
$$

We then get

$$
\begin{aligned}
R_{N, H L, 1}^{\varepsilon, 1, z} \lesssim & \left.\left.\sum_{M \geq 8} \sum_{k, l \neq 0} \int_{\eta, \xi, \xi^{\prime}} \chi|l| \geq 16|\xi|\left|A^{\sigma} \overline{\hat{f}}_{k}(\eta)\right| \chi_{N}(l, \xi) \mid \widehat{\left[1-v^{\prime}\right.}\right)\left(\xi^{\prime}-\xi\right)\right]_{M} \\
& \times\left[\frac{\langle l\rangle^{\sigma+1}}{l^{2}+\left(\xi^{\prime}-l t\right)^{2}} \widehat{\Delta_{L} \Delta_{t}^{-1} f_{l}}\left(\xi^{\prime}\right)_{<M / 8}\right]_{\sim N} \widehat{\nabla f}_{k-l}(\eta-\xi)_{<N / 8} \mid d \eta d \xi d \xi^{\prime} \\
\lesssim & \langle t\rangle^{-2} \sum_{M \geq 8}\left\|A^{\sigma} f_{\sim N}\right\|_{2} M^{-2}\left\|\left(v^{\prime}-1\right)_{M}\right\|_{H^{5}}\left\|\left(\Delta_{L} \Delta_{t}^{-1} P_{\neq} f\right)_{\sim N}\right\|_{H^{\sigma}}\|f\|_{H^{3}}
\end{aligned}
$$

which gives

$$
\begin{aligned}
R_{N, H L, 1}^{\varepsilon, 1, z} & \lesssim\langle t\rangle^{-2}\left\|A^{\sigma} f_{\sim N}\right\|_{2}\|h\|_{H^{5}}\left\|A^{\sigma}\left(\Delta_{L} \Delta_{t}^{-1} P_{\neq} f\right)_{\sim N}\right\|_{2}\|f\|_{H^{3}} \\
& \lesssim \frac{\varepsilon^{2} v^{\frac{2}{3}}}{\langle t\rangle^{2}}\left\|A^{\sigma} f_{\sim N}\right\|_{2}\left\|A^{\sigma}\left(\Delta_{L} \Delta_{t}^{-1} P_{\neq} f\right)_{\sim N}\right\|_{2} .
\end{aligned}
$$


Next we turn to $R_{N, H L, 2}^{\varepsilon, 1, z}$. If $\frac{1}{16}|\xi| \leq|l| \leq 16|\xi|$, then $|l| \approx|l, \xi| \approx\left|\xi-\xi^{\prime}\right| \approx M \approx N$,

$$
\begin{aligned}
R_{N, H L, 2}^{\varepsilon, 1, z} & \lesssim \sum_{M \geq 8}\left\|A^{\sigma} f_{\sim N}\right\|_{2} M^{-1}\left\|\left(1-v^{\prime}\right)_{M \sim N}\right\|_{H^{\sigma-1}}\left\|P_{\neq} \phi\right\|_{H^{4}}\|f\|_{H^{3}} \\
& \lesssim\left\|A^{\sigma} f_{\sim N}\right\|_{2}\left\|h_{\sim N}\right\|_{H^{\sigma-1}}\left\|P_{\neq} \phi\right\|_{H^{4}}\|f\|_{H^{3}} \\
& \lesssim \frac{\varepsilon^{2} v^{\frac{2}{3}}}{\langle t\rangle^{2}}\left\|A^{\sigma} f_{\sim N}\right\|_{2}\left\|h_{\sim N}\right\|_{H^{\sigma-1}}
\end{aligned}
$$

Next we turn to $R_{N, H L}^{\varepsilon, 1, v}$, in which case we can consider all of the "derivatives" to be landing on $1-v^{\prime}$. On the support of the integrand,

$$
\begin{gathered}
|| k, \eta|-| l, \xi|| \leq|k-l, \xi-\eta| \leq \frac{3}{16}|l, \xi|, \\
|| \xi-\xi^{\prime}|-| l, \xi|| \leq\left|l, \xi^{\prime}\right| \leq \frac{|\xi|}{16}+\left|\xi^{\prime}\right| \leq \frac{67}{100}\left|\xi^{\prime}-\xi\right| .
\end{gathered}
$$

Since $|l, \xi| \approx\left|\xi-\xi^{\prime}\right|$, the sum only includes boundedly many terms. Therefore,

$$
\begin{aligned}
R_{N, H L}^{\varepsilon, 1, v} & \lesssim\left\|A^{\sigma} f_{\sim N}\right\|_{2}\left\|\partial_{v} h_{\sim N}\right\|_{H^{\sigma-1}}\left\|\Delta_{L} \Delta_{t}^{-1} f_{\neq}\right\|_{H^{4}}\|f\|_{H^{3}} \\
& \lesssim \frac{\varepsilon^{2} v^{\frac{2}{3}}}{\left\langle v t^{3}\right\rangle}\left\|A^{\sigma} f_{\sim N}\right\|_{2}\left\|\partial_{v} h_{\sim N}\right\|_{H^{\sigma-1}} .
\end{aligned}
$$

We turn to the remainder term $R_{N, H H}^{\varepsilon, 1}$. In this case we have

$$
\left|\xi-\xi^{\prime}\right| \approx\left|\xi^{\prime}\right| \approx M \approx M^{\prime}
$$

Thus we divide into two cases according to the relationship between $l$ and $\xi^{\prime}$ :

$$
\begin{aligned}
\left|R_{N, H H}^{\varepsilon, 1}\right| \lesssim & \sum_{M \in \mathbb{D}} \sum_{\frac{1}{8} M \leq M^{\prime} \leq M} \sum_{k, l \neq 0} \int_{\eta, \xi, \xi^{\prime}} 1_{|l| \geq 3\left|\xi^{\prime}\right|} A^{\sigma} \overline{\hat{f}}_{k}(\eta) \chi_{N}(l, \xi)\left[\widehat{\left(1-v^{\prime}\right)}\left(\xi^{\prime}-\xi\right)\right]_{M^{\prime}} \\
& \times \frac{|l| A_{l}^{\sigma}(\xi)}{l^{2}+\left(\xi^{\prime}-l t\right)^{2}}\left|\widehat{\Delta_{L} \Delta_{t}^{-1}} f_{l}\left(\xi^{\prime}\right)_{M}\right|\left|\widehat{\nabla f}_{k-l}(\eta-\xi)_{<N / 8}\right| d \eta d \xi d \xi^{\prime} \\
+ & \left.\sum_{M \in \mathbb{D} \frac{1}{8} M \leq M^{\prime} \leq M} \sum_{k, l \neq 0} \int_{\eta, \xi, \xi^{\prime}} 1_{|l|<3\left|\xi^{\prime}\right|} A^{\sigma} \overline{\hat{f}}_{k}(\eta) \chi_{N}(l, \xi)\left[\widehat{\left(1-v^{\prime}\right.}\right)\left(\xi^{\prime}-\xi\right)\right]_{M^{\prime}} \\
& \times \frac{|l| A_{l}^{\sigma}(\xi)}{l^{2}+\left(\xi^{\prime}-l t\right)^{2}} \mid \widehat{\Delta_{L} \Delta_{t}^{-1} f_{l}}\left(\xi^{\prime}\right)_{M} \widehat{\nabla f} \\
= & R_{N, l}^{\varepsilon, 1, z}(\eta-\xi)_{<N / 8} \mid d \eta d \xi d \xi^{\prime} \\
& +R_{N, H H}^{\varepsilon, 1, v} .
\end{aligned}
$$

First, we consider $R_{N, H H}^{\varepsilon, 1, z}$. In this case we have $\left\langle\xi^{\prime}-\xi\right\rangle \approx\left\langle\xi^{\prime}\right\rangle$,

$$
A_{k}^{\sigma}(\eta) \approx A_{l}^{\sigma}(\xi) \approx\langle l, \xi\rangle^{\sigma} \approx\langle l\rangle^{\sigma}+\langle\xi\rangle^{\sigma} \lesssim\langle l\rangle^{\sigma}+\left\langle\xi^{\prime}\right\rangle^{\sigma}+\left\langle\xi^{\prime}-\xi\right\rangle^{\sigma} \lesssim\langle l\rangle^{\sigma},
$$

and $N \approx|k, \eta| \approx\langle l\rangle ;$ then

$$
\frac{|l| A_{l}^{\sigma}(\xi)}{l^{2}+\left(\xi^{\prime}-l t\right)^{2}} \lesssim \frac{\langle l\rangle^{\sigma-1}}{\langle t\rangle^{2}}
$$


Therefore, we get

$$
\begin{aligned}
R_{N, H H}^{\varepsilon, 1, z} & \lesssim\langle t\rangle^{-2} \sum_{M \in \mathbb{D}}\left\|A^{\sigma} f_{\sim N}\right\|_{2} M^{-2}\left\|h_{\sim M}\right\|_{H^{4}}\left\|\Delta_{L} \Delta_{t}^{-1} f_{\sim N}\right\|_{H^{\sigma}}\|f\|_{H^{3}} \\
& \lesssim\langle t\rangle^{-2}\left\|A^{\sigma} f_{\sim N}\right\|_{2}\|h\|_{H^{4}}\left\|\Delta_{L} \Delta_{t}^{-1} P_{\neq} f_{\sim N}\right\|_{H^{\sigma}}\|f\|_{H^{3}} \\
& \lesssim \frac{\varepsilon^{2} v^{\frac{2}{3}}}{\langle t\rangle^{2}}\left\|A^{\sigma} f_{\sim N}\right\|_{2}\left\|\Delta_{L} \Delta_{t}^{-1} P_{\neq} f_{\sim N}\right\|_{H^{\sigma}} .
\end{aligned}
$$

For $|l|<3\left|\xi^{\prime}\right|$, we have $\left\langle\xi^{\prime}-\xi\right\rangle \approx\left\langle\xi^{\prime}\right\rangle$,

$$
A_{k}^{\sigma}(\eta) \approx A_{l}^{\sigma}(\xi) \approx\langle l, \xi\rangle^{\sigma} \approx\langle l\rangle^{\sigma}+\langle\xi\rangle^{\sigma} \lesssim\langle l\rangle^{\sigma}+\left\langle\xi^{\prime}\right\rangle^{\sigma}+\left\langle\xi^{\prime}-\xi\right\rangle^{\sigma} \lesssim\left\langle\xi^{\prime}\right\rangle^{\sigma},
$$

and $N \approx\langle l, \xi\rangle \approx\left\langle\xi^{\prime}-\xi\right\rangle$. Thus,

$$
\begin{aligned}
R_{N, H H}^{\varepsilon, 1, v} \lesssim & \sum_{M \in \mathbb{D}} \sum_{\frac{1}{8}} \sum_{M \leq M^{\prime} \leq M} \int_{k, l \neq 0} 1_{\eta, \xi, \xi^{\prime}} 1_{|l|<3\left|\xi^{\prime}\right|} A^{\sigma} \overline{\hat{f}}_{k}(\eta) \chi_{N}(l, \xi) \\
& \times\left[\left\langle\xi^{\prime}-\xi\right\rangle^{\sigma-1} \widehat{\left(1-v^{\prime}\right)}\left(\xi^{\prime}-\xi\right)\right]_{M^{\prime}}\left\langle\xi^{\prime}\right\rangle^{2}\left|\widehat{\phi}_{l}\left(\xi^{\prime}\right)_{M} \widehat{\nabla f}_{k-l}(\eta-\xi)_{<N / 8}\right| d \eta d \xi d \xi^{\prime} \\
& \lesssim \sum_{M \in \mathbb{D}}\left\|A^{\sigma} f_{\sim N}\right\|_{2} M^{-2}\left\|h_{\sim N}\right\|_{H^{\sigma-1}}\left\|\left(\Delta_{L} \Delta_{t}^{-1} f_{\neq}\right)_{\sim M}\right\|_{H^{4}}\|f\|_{H^{3}} \\
\lesssim & \left\|A^{\sigma} f_{\sim N}\right\|_{2}\left\|h_{\sim N}\right\|_{H^{\sigma-1}}\left\|P_{\neq} \phi\right\|_{H^{4}}\|f\|_{H^{3}} \\
\lesssim & \frac{\varepsilon^{2} v^{\frac{2}{3}}}{\langle t\rangle^{2}}\left\|A^{\sigma} f_{\sim N}\right\|_{2}\left\|h_{\sim N}\right\|_{H^{\sigma-1}} .
\end{aligned}
$$

Therefore, we conclude by the bootstrap hypotheses and Lemma 4.1 that

$$
\begin{gathered}
\left|R_{N}^{1, \varepsilon}\right| \lesssim \varepsilon v^{\frac{1}{3}}\left[\left(\frac{\varepsilon v^{\frac{1}{3}}}{\left\langle t^{\frac{3}{2}} v^{\frac{1}{2}}\right\rangle}+\frac{\varepsilon v^{\frac{1}{3}}}{\left\langle t^{2}\right\rangle}+\frac{\varepsilon v^{\frac{1}{3}}}{\left\langle t^{3} v\right\rangle}\right)\left\|A^{\sigma} f_{\sim N}\right\|_{L^{2}}\left\|A^{\sigma} \Delta_{L} \Delta_{t}^{-1} P_{\neq} f_{N}\right\|_{L^{2}}\right. \\
\left.+\varepsilon\left\|\sqrt{\frac{\partial_{t} w}{w}} A^{\sigma} f_{\sim N}\right\|\left\|_{2}\right\| \sqrt{\frac{\partial_{t} w}{w}} A^{\sigma} \chi_{R} \Delta_{L} \Delta_{t}^{-1} P_{\neq} f_{N} \|_{2}\right] \\
+\frac{\varepsilon^{2} v^{\frac{2}{3}}}{\langle t\rangle^{2}}\left\|A^{\sigma} f_{\sim N}\right\|_{2}\left[\left\|\partial_{v} h_{\sim N}\right\|_{H^{\sigma-1}}+\left\|A^{\sigma}\left(\Delta_{L} \Delta_{t}^{-1} P_{\neq} f_{\neq}\right)_{\sim N}\right\|_{2}\right. \\
\left.+\left\|h_{\sim N}\right\|_{H^{\sigma-1}}\right] .
\end{gathered}
$$

We end this section by proving Proposition 2.8 .

Proof of Proposition 2.8. By (8.19), (8.20), (8.21), (8.23), Lemma 4.1, Proposition 2.3, (A.2) and the bootstrap hypotheses, we get

$$
\begin{aligned}
\sum_{N \geq 8}\left|R_{N}\right| \lesssim & \left(\frac{\varepsilon v^{\frac{1}{3}}}{\left\langle v^{\frac{1}{2}} t^{\frac{3}{2}}\right\rangle}+\frac{\varepsilon v^{\frac{1}{3}}}{\left\langle t^{2}\right\rangle}+\frac{\varepsilon v^{\frac{1}{3}}}{\left\langle v t^{3}\right\rangle}\right)\|f\|_{H^{\sigma}}\left\|A^{\sigma}\left(\Delta_{L} \Delta_{t}^{-1} P_{\neq} f\right)\right\|_{2} \\
& +\varepsilon\left\|\sqrt{\frac{\partial_{t} w}{w}} A^{\sigma} f\right\|_{2}\left\|\sqrt{\frac{\partial_{t} w}{w}} A^{\sigma} \chi_{R} \Delta_{L} \Delta_{t}^{-1} P_{\neq} f_{N}\right\|_{2}+\varepsilon v^{\frac{1}{3}}\left\|A^{\sigma} f\right\|_{2}\|g\|_{H^{\sigma}} \\
& +\frac{\varepsilon^{2} v^{\frac{2}{3}}}{\langle t\rangle^{2}}\left\|A^{\sigma} f\right\|_{2}\left[\left\|\partial_{v} h\right\|_{H^{\sigma-1}}+\left\|A^{\sigma}\left(\Delta_{L} \Delta_{t}^{-1} P_{\neq} f\right)\right\|_{2}+\|h\|_{H^{\sigma-1}}\right] .
\end{aligned}
$$


By Lemma 4.3 we have

$$
\begin{aligned}
\sum_{N \geq 8}\left|R_{N}\right| \lesssim & \left(\frac{\varepsilon v^{\frac{1}{3}}}{\left\langle v^{\frac{1}{2}} t^{\frac{3}{2}}\right\rangle}+\frac{\varepsilon v^{\frac{1}{3}}}{\left\langle t^{2}\right\rangle}+\frac{\varepsilon v^{\frac{1}{3}}}{\left\langle v t^{3}\right\rangle}\right)\|f\|_{H^{\sigma}}\left(\left\|f_{\neq}\right\|_{H^{\sigma}}+\frac{\varepsilon v^{\frac{1}{3}}}{\langle t\rangle\left\langle v t^{3}\right\rangle}\left\|\partial_{v} h\right\|_{H^{\sigma}}\right) \\
& +\varepsilon\left\|\sqrt{\frac{\partial_{t} w}{w}} A^{\sigma} f\right\|_{2}\left(\left\|\sqrt{\frac{\partial_{t} w}{w}} f_{\neq}\right\| \|_{H^{\sigma}}+\frac{\varepsilon^{2} v^{\frac{1}{2}}}{\left\langle v t^{3}\right\rangle}\right) \\
& +\varepsilon v^{\frac{1}{3}}\left\|A^{\sigma} f\right\|_{2}\|g\|_{H^{\sigma}}+\frac{\varepsilon^{2} v^{\frac{2}{3}}}{\langle t\rangle^{2}}\left\|A^{\sigma} f\right\|_{2}\left[\left\|\partial_{v} h\right\|_{H^{\sigma-1}}+\|h\|_{H^{\sigma-1}}\right],
\end{aligned}
$$

and then by the bootstrap hypotheses and the Young's inequality, it holds that

$$
\begin{aligned}
\int_{1}^{t} \sum_{N \geq 8}\left|R_{N}\left(t^{\prime}\right)\right| d t^{\prime} \lesssim & \varepsilon \sup _{t^{\prime} \in[1, t]}\left\|A^{\sigma} f\left(t^{\prime}\right)\right\|_{2}^{2}+\varepsilon \int_{1}^{t} \mathrm{CK}_{w}\left(t^{\prime}\right) d t^{\prime} \\
& +\varepsilon v^{\frac{1}{3}} \sup _{t^{\prime} \in[1, t]}\left\|A^{\sigma} f\left(t^{\prime}\right)\right\|_{2}\left\|\partial_{v} h\right\|_{L_{T}^{2}\left(H^{\sigma}\right)}\left(\int_{1}^{t}\left(\frac{\varepsilon v^{\frac{1}{3}}}{\left\langle t^{\prime}\right\rangle\left\langle v t^{\prime 3}\right\rangle}\right)^{2} d t^{\prime}\right)^{\frac{1}{2}} \\
& +\varepsilon\left(\int_{1}^{t} \mathrm{CK}_{w}\left(t^{\prime}\right) d t^{\prime}\right)^{\frac{1}{2}}\left(\int_{1}^{t}\left(\frac{\varepsilon^{2} v^{\frac{1}{2}}}{\left\langle v t^{\prime 3}\right\rangle}\right)^{2} d t^{\prime}\right)^{\frac{1}{2}} \\
& +\varepsilon v^{\frac{1}{3}} \sup _{t^{\prime} \in[1, t]}\left\|A^{\sigma} f\left(t^{\prime}\right)\right\|_{2}\|g\|_{L_{T}^{1}\left(H^{\sigma}\right)}+\varepsilon^{3} v \sup _{t^{\prime} \in[1, t]}\left\|A^{\sigma} f\left(t^{\prime}\right)\right\|_{2} \\
& +\varepsilon^{2} v^{\frac{2}{3}} \sup _{t^{\prime} \in[1, t]}\left\|A^{\sigma} f\left(t^{\prime}\right)\right\|_{2}\left\|\partial_{v} h\right\|_{L_{T}^{2}\left(H^{\sigma-1}\right)} \\
\lesssim & \varepsilon \sup _{t^{\prime} \in[1, t]}\left\|A^{\sigma} f\left(t^{\prime}\right)\right\|_{2}^{2}+\varepsilon \int_{1}^{t} \mathrm{CK}_{w}\left(t^{\prime}\right) d t^{\prime} \\
& +\varepsilon^{3} v^{\frac{1}{3}} \sup _{t^{\prime} \in[1, t]}\left\|A^{\sigma} f\left(t^{\prime}\right)\right\|_{2}+\varepsilon^{3} v^{\frac{1}{3}}\left(\int_{1}^{t} \mathrm{CK}_{w}\left(t^{\prime}\right) d t^{\prime}\right)^{\frac{1}{2}} \\
\lesssim & \varepsilon \sup _{t^{\prime} \in[1, t]}\left\|A^{\sigma} f\left(t^{\prime}\right)\right\|_{2}^{2}+\varepsilon \int_{1}^{t} \mathrm{CK}_{w}\left(t^{\prime}\right) d t^{\prime}+\varepsilon^{5} v^{\frac{2}{3}} .
\end{aligned}
$$

Thus we have proved Proposition 2.8.

\section{Coordinate system}

\subsection{Higher regular controls}

In this subsection we will study the energy estimate for $g$ in $H^{\sigma}$ and $h, \bar{h}$ in $H^{\sigma-1}$ and $H^{\sigma}$.

9.1.1. Energy estimate of $\boldsymbol{g}$. In this section we will prove (2.31). We need to mention that the result (2.31) is not optimal; however it is enough. It is natural to compute the time 
evolution of $\|g\|_{H^{\sigma}}^{2}$. We get

$$
\begin{aligned}
\frac{d}{d t}\left\|\left\langle\partial_{v}\right\rangle^{\sigma} g\right\|_{2}^{2}= & 2 \int\left\langle\partial_{v}\right\rangle^{\sigma} g\left\langle\partial_{v}\right\rangle^{\sigma} \partial_{t} g d v \\
= & -\frac{4}{t}\left\|\left\langle\partial_{v}\right\rangle^{\sigma} g\right\|_{2}^{2}-2 \int\left\langle\partial_{v}\right\rangle^{\sigma} g\left\langle\partial_{v}\right\rangle^{\sigma}\left(g \partial_{v} g\right) d v \\
& -\frac{2}{t} \int\left\langle\partial_{v}\right\rangle^{\sigma} g\left\langle\partial_{v}\right\rangle^{\sigma}\left(v^{\prime}\left\langle\nabla_{z, v}^{\perp} P_{\neq} \phi \cdot \nabla_{z, v} \tilde{u}\right\rangle\right) d v \\
& +2 v \int\left\langle\partial_{v}\right\rangle^{\sigma} g\left\langle\partial_{v}\right\rangle^{\sigma}\left(\left(v^{\prime}\right)^{2} \partial_{v}^{2} g\right) d v \\
= & -\frac{4}{t}\left\|\left\langle\partial_{v}\right\rangle^{\sigma} g\right\|_{2}^{2}+V_{1}^{H, g}+V_{2}^{H, g}+V_{3}^{H, g} .
\end{aligned}
$$

To treat $V_{1}^{H, g}$, we get by using integration by parts,

$$
\left.\left|V_{1}^{H, g}\right| \lesssim\left|\int\right| \partial_{v} g||\left\langle\partial_{v}\right\rangle^{\sigma} g\right|^{2} d v \mid+\|g\|_{H^{\sigma}}\left\|\left[\left\langle\partial_{v}\right\rangle^{\sigma}, g\right] \partial_{v} g\right\|_{2} .
$$

By Lemma A.1 and the Sobolev embedding theory, we get

$$
\left|V_{1}^{H, g}\right| \lesssim\|g\|_{H^{2}}\left\|\left\langle\partial_{v}\right\rangle^{\sigma} g\right\|_{2}^{2} .
$$

Next we treat $V_{2}^{H, g}$. We now use the fact that

$$
\left\langle\nabla_{z, v}^{\perp} P_{\neq} \phi \cdot \nabla_{z, v} \tilde{u}\right\rangle=\left\langle\nabla_{L}^{\perp} P_{\neq} \phi \cdot \nabla_{L} \tilde{u}_{\neq}\right\rangle .
$$

Then by the bootstrap hypotheses, we get

$$
\begin{aligned}
\left|V_{2}^{H, g}\right| \lesssim & \frac{2}{t} \int\left\langle\partial_{v}\right\rangle^{\sigma} g\left\langle\partial_{v}\right\rangle^{\sigma}\left[\left(v^{\prime}-1\right)\left\langle\nabla^{\perp} P_{\neq} \phi \cdot \nabla \tilde{u}_{\neq}\right\rangle\right] d v \\
& +\frac{2}{t} \int\left\langle\partial_{v}\right\rangle^{\sigma} g\left\langle\partial_{v}\right\rangle^{\sigma}\left[\left\langle\nabla_{L}^{\perp} P_{\neq} \phi \cdot \nabla_{L} \tilde{u}_{\neq}\right\rangle\right] d v \\
\lesssim & \frac{1}{t}\left\|\left\langle\partial_{v}\right\rangle^{\sigma} g\right\|_{2}\left\|\left\langle\partial_{v}\right\rangle^{\sigma}\left(v^{\prime}-1\right)\right\|_{2}\left\|\left\langle\nabla^{\perp} P_{\neq} \phi \cdot \nabla \tilde{u}_{\neq}\right\rangle\right\|_{H^{\sigma}} \\
& +\frac{1}{t}\left\|\left\langle\partial_{v}\right\rangle^{\sigma} g\right\|_{2}\left\|\left\langle\partial_{v}\right\rangle^{\sigma}\left\langle\nabla_{L}^{\perp} P_{\neq} \phi \cdot \nabla_{L} \tilde{u}_{\neq}\right\rangle\right\|_{2} \\
\lesssim & \frac{1}{t}\left\|\left\langle\partial_{v}\right\rangle^{\sigma} g\right\|_{2}\|h\|_{H^{\sigma}}\left\|\nabla_{L}^{\perp} P_{\neq} \phi\right\|_{H^{\sigma}}\left\|\nabla_{L} \tilde{u}_{\neq}\right\|_{H^{2}} \\
& +\frac{1}{t}\left\|\left\langle\partial_{v}\right\rangle^{\sigma} g\right\|_{2}\|h\|_{H^{\sigma}}\left\|\nabla_{L}^{\perp} P_{\neq} \phi\right\|_{H^{2}}\left\|\nabla_{L} \tilde{u}_{\neq}\right\|_{H^{\sigma}} \\
& +\frac{1}{t}\left\|\left\langle\partial_{v}\right\rangle^{\sigma} g\right\|_{2}\left\|\nabla_{L}^{\perp} P_{\neq} \phi\right\|_{H^{\sigma}}\left\|\nabla_{L} \tilde{u}_{\neq}\right\|_{H^{2}} \\
& +\frac{1}{t}\left\|\left\langle\partial_{v}\right\rangle^{\sigma} g\right\|_{2}\left\|\nabla_{L}^{\perp} P_{\neq} \phi\right\|_{H^{2}}\left\|\nabla_{L} \tilde{u}_{\neq}\right\|_{H^{\sigma}} \\
\lesssim & \frac{1}{t}\left\|\left\langle\partial_{v}\right\rangle^{\sigma} g\right\|_{2}\left\|\nabla_{L}^{\perp} P_{\neq} \phi\right\|_{H^{\sigma}}\left\|\nabla_{L} \tilde{u}_{\neq}\right\|_{H^{2}}+\frac{1}{t}\left\|\left\langle\partial_{v}\right\rangle^{\sigma} g\right\|_{2}\left\|\nabla_{L}^{\perp} P_{\neq} \phi\right\|_{H^{2}}\left\|\nabla_{L} \tilde{u}_{\neq}\right\|_{H^{\sigma}}
\end{aligned}
$$


Finally, we treat the dissipation term $V_{3}^{H, g}$. We have

$$
\begin{aligned}
V_{3}^{H, g} & =2 v \int\left\langle\partial_{v}\right\rangle^{\sigma} g\left\langle\partial_{v}\right\rangle^{\sigma}\left(\partial_{v}^{2} g\right) d v+2 v \int\left\langle\partial_{v}\right\rangle^{\sigma} g\left\langle\partial_{v}\right\rangle^{\sigma}\left(\left(\left(v^{\prime}\right)^{2}-1\right) \partial_{v}^{2} g\right) d v \\
& =-2 v\left\|\partial_{v}\left\langle\partial_{v}\right\rangle^{\sigma} g\right\|_{2}^{2}+2 v \int\left\langle\partial_{v}\right\rangle^{\sigma} g\left\langle\partial_{v}\right\rangle^{\sigma}\left(\left(\left(v^{\prime}\right)^{2}-1\right) \partial_{v}^{2} g\right) d v \\
& =-2 v\left\|\partial_{v}\left\langle\partial_{v}\right\rangle^{\sigma} g\right\|_{2}^{2}+V_{3, \varepsilon}^{H, g} .
\end{aligned}
$$

The term $V_{3, \varepsilon}^{H, g}$ is similar to $E^{0}$. We then obtain by Young's inequality that

$$
\begin{aligned}
\left|V_{3, \varepsilon}^{H, g}\right| & \lesssim v\left(1+\|h\|_{H^{2}}\right)\left(\left\|\partial_{v} g\right\|_{H^{\sigma}}^{2}\|h\|_{H^{2}}+\left\|\partial_{v} h\right\|_{H^{\sigma-2}}\left\|\partial_{v} g\right\|_{H^{\sigma}}\|g\|_{H^{4}}\right) \\
& \lesssim v\left(1+\|h\|_{H^{2}}\right)\left(\left\|\partial_{v} g\right\|_{H^{\sigma}}^{2}\|h\|_{H^{2}}+\|h\|_{H^{\sigma-1}}\left\|\partial_{v} g\right\|_{H^{\sigma}}^{2}+\left\|\partial_{v} h\right\|_{H^{\sigma-2}}\|g\|_{H^{4}}^{2}\right) .
\end{aligned}
$$

By the bootstrap assumption, we get

$$
\begin{aligned}
\frac{d}{d t}\|g\|_{H^{\sigma}}^{2} \leq & -\frac{4}{t}\|g\|_{H^{\sigma}}^{2}-v\left\|\partial_{v} g\right\|_{H^{\sigma}}^{2}+C\left(\|g\|_{H^{2}}\|g\|_{H^{\sigma}}^{2}\right. \\
& +\frac{1}{t}\|g\|_{H^{\sigma}}\left(\left\|\nabla_{L}^{\perp} P_{\neq} \phi\right\|_{H^{\sigma}}\left\|\nabla_{L} \tilde{u}_{\neq}\right\|_{H^{2}}+\left\|\nabla_{L}^{\perp} P_{\neq} \phi\right\|_{H^{2}}\left\|\nabla_{L} \tilde{u}_{\neq}\right\|_{H^{\sigma}}\right) \\
& \left.+v\left\|\partial_{v} h\right\|_{H^{\sigma-2}}\|g\|_{H^{4}}^{2}\right) \\
\leq & -\frac{4}{t}\|g\|_{H^{\sigma}}^{2}+C\left[\|g\|_{H^{2}}\|g\|_{H^{\sigma}}^{2}+v\left\|\partial_{v} h\right\|_{H^{\sigma-2}}\|g\|_{H^{\sigma}}\|g\|_{H^{4}}\right. \\
& \left.+\frac{1}{t}\|g\|_{H^{\sigma}}\left(\left\|\Delta_{L} P_{\neq} \phi\right\|_{H^{\sigma}}\left\|\nabla_{L} \tilde{u}_{\neq}\right\|_{H^{2}}+\left\|\nabla_{L}^{\perp} P_{\neq} \phi\right\|_{H^{2}}\left\|\nabla_{L} \tilde{u}_{\neq}\right\|_{H^{\sigma}}\right)\right],
\end{aligned}
$$

which gives that for $t \geq 1$,

$$
\begin{aligned}
& \sup _{t^{\prime} \in[1, t]}\left(t^{\prime}\left\|g\left(t^{\prime}\right)\right\|_{H^{\sigma}}\right)+\int_{1}^{t}\left\|g\left(t^{\prime}\right)\right\|_{H^{\sigma}} d t^{\prime}
\end{aligned}
$$

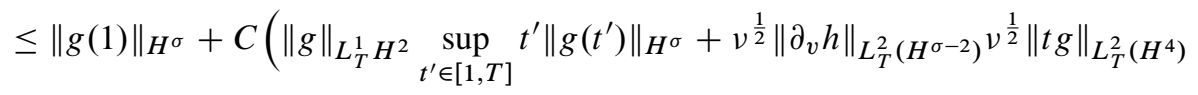

$$
\begin{aligned}
& +\left\|f_{\neq}\right\|_{L_{T}^{\infty}\left(H^{\sigma}\right)}\left\|\nabla_{L} \tilde{u}_{\neq}\right\|_{L_{T}^{1} H^{2}}+\left\|\frac{\varepsilon v^{\frac{1}{3}}}{\langle t\rangle\left\langle v t^{3}\right\rangle}\right\| \partial_{v} h\left\|_{H^{\sigma}}\right\|_{L_{T}^{1}}\left\|\nabla_{L} \tilde{u}_{\neq}\right\|_{L_{T}^{\infty}\left(H^{2}\right)} \\
& \left.+\left\|\nabla_{L}^{\perp} P_{\neq} \phi\right\|_{L_{T}^{1}\left(H^{2}\right)}\left\|f_{\neq}\right\|_{L_{T}^{\infty} H^{\sigma}}+\left\|\nabla_{L}^{\perp} P_{\neq} \phi\right\|_{L_{T}^{\infty}\left(H^{2}\right)}\left\|\frac{\varepsilon v^{\frac{1}{3}}}{\langle t\rangle\left\langle v t^{3}\right\rangle}\right\| \partial_{v} h\left\|_{H^{\sigma}}\right\|_{L_{T}^{1}}\right) \\
& \leq\|g(1)\|_{H^{\sigma}}+C\left(\|g\|_{L_{T}^{1} H^{2}} \sup _{t^{\prime} \in[1, T]} t^{\prime}\left\|g\left(t^{\prime}\right)\right\|_{H^{\sigma}}+v^{\frac{1}{2}}\left\|\partial_{v} h\right\|_{L_{T}^{2}\left(H^{\sigma-2}\right)} v^{\frac{1}{2}}\|t g\|_{L_{T}^{2}\left(H^{4}\right)}\right. \\
& \left.+\varepsilon v^{\frac{1}{3}}\left\|\nabla_{L} \tilde{u}_{\neq}\right\|_{L_{T}^{1} H^{2}}+\varepsilon v^{\frac{1}{3}}\left\|\nabla_{L}^{\perp} P_{\neq} \phi\right\|_{L_{T}^{1}\left(H^{2}\right)}+\varepsilon^{2} v^{\frac{2}{3}}\left\|\partial_{v} h\right\|_{L_{T}^{2}\left(H^{\sigma}\right)}\right) .
\end{aligned}
$$


Thus, by the bootstrap hypotheses we get

$$
\begin{aligned}
& \sup _{t^{\prime} \in[1, t]}\left(t^{\prime}\left\|g\left(t^{\prime}\right)\right\|_{H^{\sigma}}\right)+\int_{1}^{t}\left\|g\left(t^{\prime}\right)\right\|_{H^{\sigma}} d t^{\prime} \\
& \quad \leq\|g(1)\|_{H^{\sigma}}+C\left(\varepsilon v^{\frac{1}{3}} \sup _{t^{\prime} \in[1, T]} t^{\prime}\left\|g\left(t^{\prime}\right)\right\|_{H^{\sigma}}+\varepsilon^{2} v^{\frac{1}{3}}\right) .
\end{aligned}
$$

By taking $\varepsilon$ small enough, we have proved (2.31).

\subsubsection{Energy estimate of $\overline{\boldsymbol{h}}$ and $\boldsymbol{h}$. We get}

$$
\begin{aligned}
\frac{1}{2} \frac{d}{d t}\|\bar{h}\|_{H^{\sigma-1}}^{2}= & -\int\left\langle\partial_{v}\right\rangle^{\sigma-1} \bar{h}\left\langle\partial_{v}\right\rangle^{\sigma-1}\left(g \partial_{v} \bar{h}\right) d v-\frac{2}{t}\|\bar{h}\|_{H^{\sigma-1}}^{2} \\
& +\frac{1}{t} \int\left\langle\partial_{v}\right\rangle^{\sigma-1} \bar{h}\left\langle\partial_{v}\right\rangle^{\sigma-1}\left(v^{\prime}\left\langle\nabla_{z, v}^{\perp} P_{\neq} \phi \cdot \nabla_{z, v} f_{\neq}\right\rangle\right) d v \\
& +v \int\left\langle\partial_{v}\right\rangle^{\sigma-1} \bar{h}\left\langle\partial_{v}\right\rangle^{\sigma-1}\left(\left(\left(v^{\prime}\right)^{2}-1\right) \partial_{v v} \bar{h}\right) d v-v\left\|\partial_{v} \bar{h}\right\|_{H^{\sigma-1}} \\
= & -\frac{2}{t}\|\bar{h}\|_{H^{\sigma-1}}^{2}-v\left\|\partial_{v} \bar{h}\right\|_{H^{\sigma-1}}+V_{1}^{H, \bar{h}}+V_{2}^{H, \bar{h}}+V_{3}^{H, \bar{h}}
\end{aligned}
$$

and

$$
\begin{aligned}
\frac{1}{2} \frac{d}{d t}\|h\|_{H^{\sigma-1}}^{2}= & -\int\left\langle\partial_{v}\right\rangle^{\sigma-1} h\left\langle\partial_{v}\right\rangle^{\sigma-1}\left(g \partial_{v} h-\bar{h}-v\left(v^{\prime}\right)^{2} \partial_{v}^{2} h\right) d v \\
= & -\int\left\langle\partial_{v}\right\rangle^{\sigma-1} h\left\langle\partial_{v}\right\rangle^{\sigma-1}\left(g \partial_{v} h\right) d v \\
& +\int\left\langle\partial_{v}\right\rangle^{\sigma-1} h\left\langle\partial_{v}\right\rangle^{\sigma-1} \bar{h} d v \\
& +v \int\left\langle\partial_{v}\right\rangle^{\sigma-1} h\left\langle\partial_{v}\right\rangle^{\sigma-1}\left(\left(\left(v^{\prime}\right)^{2}-1\right) \partial_{v}^{2} h\right) d v-v\left\|\partial_{v} h\right\|_{H^{\sigma-1}}^{2} \\
= & -v\left\|\partial_{v} h\right\|_{H^{\sigma-1}}^{2}+V_{1}^{H, h}+V_{2}^{H, h}+V_{3}^{H, h} .
\end{aligned}
$$

We use the same argument as in the treatment of $V_{1}^{H, g}$ and get

$$
\left|V_{1}^{H, \bar{h}}\right| \lesssim\|g\|_{H^{\sigma-1}}\|\bar{h}\|_{H^{\sigma-1}}^{2}
$$

and

$$
\left|V_{1}^{H, h}\right| \lesssim\|g\|_{H^{\sigma-1}}\|\bar{h}\|_{H^{\sigma-1}}^{2}
$$

We also have

$$
\left|V_{2}^{H, h}\right| \leq\|h\|_{H^{\sigma-1}}\|\bar{h}\|_{H^{\sigma-1}} .
$$

To treat $V_{2}^{H, \bar{h}}$ we have

$$
\begin{aligned}
V_{2}^{H, \bar{h}}= & \frac{1}{t} \int\left\langle\partial_{v}\right\rangle^{\sigma-1} \bar{h}\left\langle\partial_{v}\right\rangle^{\sigma-1}\left(\left\langle\nabla_{z, v}^{\perp} P_{\neq} \phi \cdot \nabla_{z, v} f_{\neq}\right\rangle\right) d v \\
& +\frac{1}{t} \int\left\langle\partial_{v}\right\rangle^{\sigma-1} \bar{h}\left\langle\partial_{v}\right\rangle^{\sigma-1}\left(h\left\langle\nabla_{z, v}^{\perp} P_{\neq} \phi \cdot \nabla_{z, v} f_{\neq}\right\rangle\right) d v
\end{aligned}
$$


Thus, by the fact that

$$
\left\langle-\partial_{v} F \partial_{z} G+\partial_{z} F \partial_{v} G\right\rangle=\partial_{v}\left\langle G \partial_{z} F\right\rangle,
$$

we get

$$
\begin{aligned}
\mid V_{2}^{H, \bar{h} \mid \lesssim} & \frac{1}{t}\|\bar{h}\|_{H^{\sigma-1}}\left(1+\|h\|_{H^{2}}\right)\left\|\left\langle\nabla_{z, v}^{\perp} P_{\neq} \phi \cdot \nabla_{z, v} f_{\neq}\right\rangle\right\|_{H^{\sigma-1}} \\
& +\frac{1}{t}\|\bar{h}\|_{H^{\sigma-1}}\|h\|_{H^{\sigma-1}}\left\|\left\langle\nabla_{z, v}^{\perp} P_{\neq} \phi \cdot \nabla_{z, v} f_{\neq}\right\rangle\right\|_{H^{1}} \\
\lesssim & \frac{1}{t}\|\bar{h}\|_{H^{\sigma-1}}\left(1+\|h\|_{H^{2}}\right)\left\|\left\langle\partial_{z} P_{\neq} \phi f_{\neq}\right\rangle\right\|_{H^{\sigma}} \\
& +\frac{1}{t}\|\bar{h}\|_{H^{\sigma-1}}\|h\|_{H^{\sigma-1}}\left\|\left\langle\partial_{z} P_{\neq} \phi f_{\neq}\right\rangle\right\|_{H^{2}} \\
\lesssim & \frac{1}{t}\|\bar{h}\|_{H^{\sigma-1}}\left(1+\|h\|_{H^{2}}\right)\left\|P_{\neq} \phi\right\|_{H^{\sigma}}\left\|\partial_{z} f_{\neq}\right\|_{H^{1}} \\
& +\frac{1}{t}\|\bar{h}\|_{H^{\sigma-1}}\left(1+\|h\|_{H^{2}}\right)\left\|\partial_{z} P_{\neq} \phi\right\|_{H^{1}}\left\|f_{\neq}\right\|_{H^{\sigma}} \\
& +\frac{1}{t}\|\bar{h}\|_{H^{\sigma-1}}\|h\|_{H^{\sigma-1}}\left\|\partial_{z} P_{\neq} \phi\right\|_{H^{2}}\left\|f_{\neq}\right\|_{H^{2}} .
\end{aligned}
$$

Next we turn to $V_{3}^{H, \bar{h}}$ and $V_{3}^{H, h}$ :

$$
\begin{aligned}
\left|V_{3, \varepsilon}^{H, \bar{h}}\right| \lesssim & \left.\int_{\xi, \eta}\langle\eta\rangle^{2 \sigma-2}|\hat{\bar{h}}(\eta)| \mid \widehat{\left(1-v^{\prime}\right)^{2}}(\eta-\xi)\right)|\xi|^{2} \hat{\bar{h}}(\xi) \mid d \xi d \eta \\
\lesssim & \left.\int_{\xi, \eta} 1_{|\eta| \leq 1}|\hat{\bar{h}}(\eta)| \mid \widehat{\left(1-v^{\prime}\right)^{2}}(\eta-\xi)\right)|\xi|(|\eta|+|\xi-\eta|) \hat{\bar{h}}(\xi) \mid d \eta d \xi \\
& \left.+\int_{\xi, \eta} 1_{|\eta| \geq 1} 1_{|\xi-\eta| \geq|\xi|}|\eta|^{2 \sigma-2}|\hat{\bar{h}}(\eta)| \mid \widehat{\left(1-v^{\prime}\right)^{2}}(\eta-\xi)\right)|\xi|^{2} \hat{\bar{h}}(\xi) \mid d \xi d \eta \\
& \left.+\int_{\xi, \eta} 1_{|\eta| \geq 1} 1_{|\xi-\eta|<|\xi|}|\eta|^{2 \sigma-2}|\hat{\bar{h}}(\eta)| \mid \widehat{\left(1-\left(v^{\prime}\right)^{2}\right.}(\eta-\xi)\right)|\xi|^{2} \hat{\bar{h}}(\xi) \mid d \xi d \eta \\
\lesssim & \int_{\xi, \eta} 1_{|\eta| \leq 1}|\eta||\hat{\bar{h}}(\eta)|\left|\left(\widehat{\left(1-\left(v^{\prime}\right)^{2}\right)}(\eta-\xi)\right)\right| \xi\left|\hat{f_{0}}(\xi)\right| d \eta d \xi \\
& +\int_{\xi, \eta} 1_{|\eta| \leq 1}|\hat{\bar{h}}(\eta)|\left|\left(\widehat{\left(\partial_{v}\left(1-\left(v^{\prime}\right)^{2}\right)\right.}(\eta-\xi)\right)\right| \xi|\hat{\bar{h}}(\xi)| d \eta d \xi \\
& +\left.\int_{\xi, \eta} 1_{|\eta| \geq 1} 1_{|\xi-\eta|<|\xi|}|\eta|^{\sigma}|\hat{\bar{h}}(\eta)|\left|\left(\widehat{1-\left(v^{\prime}\right)^{2}}(\eta-\xi)\right)\right| \xi\right|^{\sigma} \hat{\bar{h}}(\xi) \mid d \xi d \eta \\
& \left.+\int_{\xi, \eta} 1_{|\eta| \geq 1} 1_{|\xi-\eta| \geq|\xi|}|\eta|^{\sigma-1}|\hat{\bar{h}}(\eta)| \mid \widehat{\left(1-\left(v^{\prime}\right)^{2}\right.}(\eta-\xi)\right)|\eta-\xi|^{\sigma-1}|\xi|^{2} \hat{\bar{h}}(\xi) \mid d \xi d \eta \\
\lesssim & \left\|\left(1-\left(v^{\prime}\right)^{2}\right)\right\|_{H^{2}}\left\|\partial_{v} \bar{h}\right\|_{H^{\sigma-1}}+\|\bar{h}\|_{H^{\sigma-1}}\left\|\left(1-\left(v^{\prime}\right)^{2}\right)\right\|_{H^{\sigma-1}}\|\overline{\bar{h}}\|_{H^{4}} \\
\lesssim & \left(1+\|h\|_{H^{2}}\right)\left(\|h\|_{H^{2}}\left\|\partial_{v} \bar{h}\right\|_{H^{\sigma-1}}+\|\bar{h}\|_{H^{\sigma-1}}\left\|\partial_{v} h\right\|_{H^{\sigma-2}}\|\bar{h}\|_{H^{4}}\right)
\end{aligned}
$$

and

$$
\left|V_{3, \varepsilon}^{H, h}\right| \lesssim v\left(1+\|h\|_{H^{2}}\right)\left\|\partial_{v} h\right\|_{H^{\sigma-1}}^{2}\|h\|_{H^{4}} .
$$


Putting these together and using the bootstrap assumption, we get

$$
\begin{aligned}
\frac{1}{2} \frac{d}{d t}\|\bar{h}\|_{H^{\sigma-1}}^{2} \leq-\frac{2}{t}\|\bar{h}\|_{H^{\sigma-1}}^{2}+C( & \|g\|_{H^{2}}\|\bar{h}\|_{H^{\sigma-1}}^{2} \\
& +\frac{1}{t}\|\bar{h}\|_{H^{\sigma-1}}\left(1+\|h\|_{H^{2}}\right)\left\|P_{\neq} \phi\right\|_{H^{\sigma}}\left\|\partial_{z} f_{\neq}\right\|_{H^{1}} \\
& +\frac{1}{t}\|\bar{h}\|_{H^{\sigma-1}}\left(1+\|h\|_{H^{2}}\right)\left\|\partial_{z} P_{\neq} \phi\right\|_{H^{1}}\left\|f_{\neq}\right\|_{H^{\sigma}} \\
& +\frac{1}{t}\|\bar{h}\|_{H^{\sigma-1}}\|h\|_{H^{\sigma-1}}\left\|\partial_{z} P_{\neq} \phi\right\|_{H^{2}}\left\|f_{\neq}\right\|_{H^{2}} \\
& \left.+v\left\|\partial_{v} h\right\|_{H^{\sigma-2}}\|\bar{h}\|_{H^{\sigma-1}}\|\bar{h}\|_{H^{4}}\right) .
\end{aligned}
$$

We also get

$$
\begin{aligned}
\frac{d}{d t}\left(t\|\bar{h}\|_{H^{\sigma-1}}\right) \leq-\|\bar{h}\|_{H^{\sigma-1}}+C( & t\|g\|_{H^{2}}\|\bar{h}\|_{H^{\sigma-1}}+\left\|P_{\neq} \phi\right\|_{H^{\sigma}}\left\|\partial_{z} f_{\neq}\right\|_{H^{1}} \\
& \left.+\left\|\partial_{z} P_{\neq} \phi\right\|_{H^{2}}\left\|f_{\neq}\right\|_{H^{\sigma}}+v t\left\|\partial_{v} h\right\|_{H^{\sigma-2}}\|\bar{h}\|_{H^{4}}\right),
\end{aligned}
$$

which then implies

$$
\begin{aligned}
& \sup _{t \in[1, T]}\left(t\|\bar{h}(t)\|_{H^{\sigma-1}}\right)+ \int_{0}^{T}\left\|\bar{h}\left(t^{\prime}\right)\right\|_{H^{\sigma-1}} d t^{\prime} \\
& \leq\|\bar{h}(1)\|_{H^{\sigma-1}}+C\left(\|g\|_{L_{T}^{1}\left(H^{2}\right)} \sup _{t \in[1, T]}\left(t\|\bar{h}(t)\|_{H^{\sigma-1}}\right)+\left\|P_{\neq} \phi\right\|_{L_{T}^{\infty} H^{\sigma}}\left\|\partial_{z} f_{\neq}\right\|_{L_{T}^{1} H^{1}}\right. \\
& \quad+\left\|\partial_{z} P_{\neq} \phi\right\|_{L_{T}^{1} H^{2}}\left\|f_{\neq}\right\|_{L^{\infty}\left(H^{\sigma}\right)} \\
&\left.\quad+v\left\|\partial_{v} h\right\|_{L_{T}^{2} H^{\sigma-2}}\|\langle t\rangle\| \bar{h}\left\|_{H^{4}}\right\|_{L_{T}^{2}}\right) \\
& \leq\|\bar{h}(1)\|_{H^{\sigma-1}}+C\left(\varepsilon v^{\frac{1}{3}} \sup _{t \in[1, T]}\left(t\|\bar{h}(t)\|_{H^{\sigma-1}}\right)+\varepsilon^{2} v^{\frac{1}{3}}+\varepsilon^{2} v^{\frac{7}{6}}\right) .
\end{aligned}
$$

Then by taking $\varepsilon$ small enough, we get (2.32).

We also get by Young's inequality and the bootstrap hypotheses that

$$
\begin{aligned}
& \sup _{t \in[1, T]}\left(\|h(t)\|_{H^{\sigma-1}}^{2}\right)+v\left\|\partial_{v} h\right\|_{L_{T}^{2} H^{\sigma-1}}^{2} \\
& \leq\|h(1)\|_{H^{\sigma-1}}^{2}+\|\bar{h}\|_{L_{T}^{1}\left(H^{\sigma-1}\right)} \sup _{t \in[1, T]}\left(\|h(t)\|_{H^{\sigma-1}}\right) \\
&+C\|g\|_{L_{T}^{1}\left(H^{2}\right)} \sup _{t \in[1, T]}\left(\|h(t)\|_{H^{\sigma-1}}^{2}\right) \\
& \leq\|h(1)\|_{H^{\sigma-1}}^{2}+4\|\bar{h}\|_{L_{T}^{1}\left(H^{\sigma-1}\right)}^{2}+\left(\frac{1}{4}+C\|g\|_{L_{T}^{1}\left(H^{2}\right)}\right) \sup _{t \in[1, T]}\left(\|h(t)\|_{H^{\sigma-1}}^{2}\right) \\
& \leq\|h(1)\|_{H^{\sigma-1}}^{2}+4\|\bar{h}\|_{L_{T}^{1}\left(H^{\sigma-1}\right)}^{2}+\frac{3}{8} \sup _{t \in[1, T]}\left(\|h(t)\|_{H^{\sigma-1}}^{2}\right),
\end{aligned}
$$

which implies (2.33). 


\subsection{Energy estimates of $\bar{h}$ and $h$ in $H^{\sigma}$}

We have

$$
\begin{aligned}
& \frac{1}{2} \frac{d}{d t}\left\|A^{\sigma} \bar{h}\right\|_{2}^{2} \\
& =-\int \frac{\partial_{t} w(t, \eta)}{w(t, \eta)}\left|\frac{\langle\eta\rangle^{\sigma} \hat{\bar{h}}(t, \eta)}{w(t, \eta)}\right|^{2} d \eta-\frac{2}{t}\left\|A^{\sigma} \bar{h}\right\|_{2}^{2} \\
& \underbrace{-\int A^{\sigma} \bar{h}\left[A^{\sigma}\left(g \partial_{v} \bar{h}\right)-g \partial_{v} A^{\sigma} \bar{h}\right] d v}_{V_{1, \sigma}^{\bar{h}}}+\frac{1}{2} \int g^{\prime}\left|A^{\sigma} \bar{h}\right|^{2} d v \\
& +\underbrace{\frac{1}{t} \sum_{l \neq 0} \int \frac{\langle\eta\rangle^{\sigma} \hat{\bar{h}}(t, \eta)}{w(t, \eta)} \frac{\langle\eta\rangle^{\sigma} \eta l}{w(t, \eta)} \overline{\hat{\phi}_{-l}(\eta-\xi) \hat{f}_{l}(\xi)} d \xi d \eta}_{V_{2, \sigma}^{\bar{h}}} \\
& +\underbrace{\frac{1}{t} \sum_{l \neq 0} \int \frac{\langle\eta\rangle^{\sigma} \overline{\hat{h}}(t, \eta)}{w(t, \eta)} \frac{\langle\eta\rangle^{\sigma}\left(\eta-\xi^{\prime}\right) l}{w(t, \eta)} \hat{h}\left(\xi^{\prime}\right) \hat{\phi}_{-l}(\eta-\xi) \hat{f}_{l}\left(\xi-\xi^{\prime}\right) d \xi^{\prime} d \xi d \eta}_{V_{2, \sigma}^{\bar{h}, \varepsilon}} \\
& -v\left\|\partial_{v} A^{\sigma} \bar{h}\right\|_{2}^{2}+\underbrace{v \int A^{\sigma} \bar{h} A^{\sigma}\left(\left(\left(v^{\prime}\right)^{2}-1\right) \partial_{v v} \bar{h}\right) d v}_{V_{3, \sigma}^{\bar{h}}} \\
& =-\mathrm{CK}_{w}^{\bar{h}}-\frac{2}{t}\left\|A^{\sigma} \bar{h}\right\|_{2}^{2}-v\left\|\partial_{v} A^{\sigma} \bar{h}\right\|_{2}^{2}+V_{1, \sigma}^{\bar{h}}+V_{2, \sigma}^{\bar{h}}+V_{2, \sigma}^{\bar{h}, \varepsilon}+V_{3, \sigma}^{\bar{h}} .
\end{aligned}
$$

We then get

$$
\begin{aligned}
V_{1, \sigma}^{\bar{h}}= & -\sum_{M \geq 8} \int A^{\sigma} \bar{h}\left[A^{\sigma}\left(g_{M} \partial_{v} \bar{h}_{<M / 8}\right)-g_{M} \partial_{v} A^{\sigma} \bar{h}_{<M / 8}\right] d v \\
& -\sum_{M \geq 8} \int A^{\sigma} \bar{h}\left[A^{\sigma}\left(g_{<M / 8} \partial_{v} \bar{h}_{M}\right)-g_{<M / 8} \partial_{v} A^{\sigma} \bar{h}_{M}\right] d v \\
& -\sum_{M \in \mathbb{D}} \sum_{\frac{1}{8} M \leq M^{\prime} \leq 8 M} \int A^{\sigma} \bar{h}\left[A^{\sigma}\left(g_{M} \partial_{v} \bar{h}_{M^{\prime}}\right)-g_{M} \partial_{v} A^{\sigma} \bar{h}_{M^{\prime}}\right] d v \\
= & V_{1, \sigma}^{\bar{h}, H L}+V_{1, \sigma}^{\bar{h}, L H}+V_{1, \sigma}^{\bar{h}, H H} .
\end{aligned}
$$

We have

$$
\begin{aligned}
\left|V_{1, \sigma}^{\bar{h}, H L}\right| \lesssim \sum_{M \geq 8} \int A^{\sigma} \hat{\bar{h}}(\eta)[ & A^{\sigma}\left(\hat{g}(\xi)_{M} \widehat{\partial_{v} \overline{\bar{h}}}(\eta-\xi)_{<M / 8}\right) \\
& \left.-\hat{g}(\xi)_{M} A^{\sigma} \widehat{\partial_{v} \bar{h}}(\eta-\xi)_{<M / 8}\right] d \xi d \eta
\end{aligned}
$$




$$
\begin{aligned}
& \lesssim \sum_{M \geq 8} \int A^{\sigma} \hat{\bar{h}}(\eta)\langle\xi\rangle^{\sigma} \hat{g}(\xi)_{M} \widehat{\partial_{v} \bar{h}}(\eta-\xi)_{<M / 8} d \xi d \eta \\
& \lesssim \sum_{M \geq 8}\left\|A^{\sigma} \bar{h} \sim M\right\|_{2}\left\|g_{M}\right\|_{H^{\sigma}}\left\|\partial_{v} \bar{h}\right\|_{H^{1}} \\
& \lesssim\left\|A^{\sigma} \bar{h}\right\|_{2}\|g\|_{H^{\sigma}}\left\|\partial_{v} \bar{h}\right\|_{H^{1}} .
\end{aligned}
$$

Next we treat $V_{1, \sigma}^{\bar{h}, L H}+V_{1, \sigma}$ and we get

$$
\begin{array}{r}
\left|V_{1, \sigma}^{\bar{h}, H L}\right| \lesssim \sum_{M \geq 8} \int_{|\xi-\eta| \leq \frac{1}{10}|\eta|} A^{\sigma} \hat{\bar{h}}(\eta)\left[A^{\sigma}\left(\hat{g}(\eta-\xi)_{<M / 8} \widehat{\partial_{v} \bar{h}}(\xi)_{M}\right)\right. \\
\left.-\hat{g}(\eta-\xi)_{<M / 8} A^{\sigma} \widehat{\partial_{v} \bar{h}}(\xi)_{M}\right] d \xi d \eta \\
+\sum_{M \geq 8} \int_{|\xi-\eta| \geq \frac{1}{10}|\eta|} A^{\sigma} \hat{\bar{h}}(\eta)\left[A^{\sigma}\left(\hat{g}(\eta-\xi)_{<M / 8} \widehat{\partial_{v} \bar{h}}(\xi)_{M}\right)\right. \\
\left.-\hat{g}(\eta-\xi)_{<M / 8} A^{\sigma} \widehat{\partial_{v} \bar{h}}(\xi)_{M}\right] d \xi d \eta .
\end{array}
$$

For the first term, by Lemma 6.1 we have $|\xi| \approx|\eta|$, and then

$$
\begin{aligned}
& \mid \sum_{M \geq 8} \int_{|\xi-\eta| \leq \frac{1}{10}|\eta|} A^{\sigma} \hat{\bar{h}}(\eta)\left[A^{\sigma}\left(\hat{g}(\eta-\xi)_{<M / 8} \widehat{\partial_{v} \bar{h}}(\xi)_{M}\right)\right. \\
& \left.-\hat{g}(\eta-\xi)_{<M / 8} A^{\sigma} \widehat{\partial_{v} \bar{h}}(\xi)_{M}\right] d \xi d \eta \mid \\
& \lesssim \sum_{M \geq 8} \int_{|\xi-\eta| \leq \frac{1}{10}|\eta|} A^{\sigma}|\hat{\bar{h}}(\eta)|\left|\frac{\langle\eta\rangle \eta}{w(t, \eta)}-\frac{\langle\xi\rangle \xi}{w(t, \xi)}\right| \hat{g}(\eta-\xi)_{<M / 8} \hat{\bar{h}}(\xi)_{M} \mid d \xi d \eta \\
& \lesssim \sum_{M \geq 8} \int_{|\xi-\eta| \leq \frac{1}{10}|\eta|} A^{\sigma}|\hat{\bar{h}}(\eta)|\left|\langle\eta\rangle^{\sigma} \eta-\langle\xi\rangle^{\sigma} \xi\right|\left|\hat{g}(\eta-\xi)_{<M / 8} \hat{\bar{h}}(\xi)_{M}\right| d \xi d \eta \\
& +\sum_{M \geq 8} \int_{|\xi-\eta| \leq \frac{1}{10}|\eta|} A^{\sigma}|\hat{\bar{h}}(\eta)|\left|\frac{1}{w(t, \eta)}-\frac{1}{w(t, \xi)}\right|\langle\xi\rangle^{\sigma+1}\left|\hat{g}(\eta-\xi)_{<M / 8} \hat{\bar{h}}(\xi)_{M}\right| d \xi d \eta \\
& \lesssim \sum_{M \geq 8} \int_{|\xi-\eta| \leq \frac{1}{10}|\eta|} A^{\sigma}|\hat{\bar{h}}(\eta)|\langle\eta-\xi\rangle\langle\xi\rangle^{\sigma}\left|\hat{g}(\eta-\xi)_{<M / 8} \hat{\bar{h}}(\xi)_{M}\right| d \xi d \eta \\
& +\sum_{M \geq 8} \int_{|\xi-\eta| \leq \frac{1}{10}|\eta|} A^{\sigma}|\hat{\bar{h}}(\eta)|\left|\frac{1}{w(t, \eta)}-\frac{1}{w(t, \xi)}\right|\langle\xi\rangle^{\sigma+1}\left|\hat{g}(\eta-\xi)_{<M / 8} \hat{\bar{h}}(\xi)_{M}\right| d \xi d \eta \\
& \lesssim \sum_{M \geq 8} \int_{|\xi-\eta| \leq \frac{1}{10}|\eta|} A^{\sigma}|\hat{\bar{h}}(\eta)|\langle\eta-\xi\rangle\langle\xi\rangle^{\sigma}\left|\hat{g}(\eta-\xi)_{<M / 8} \hat{\bar{h}}(\xi)_{M}\right| d \xi d \eta \\
& +\sum_{M \geq 8} \int_{|\xi-\eta| \leq \frac{1}{10}|\eta|} A^{\sigma}|\hat{\bar{h}}(\eta)| \frac{\langle\eta-\xi\rangle}{\eta}\left(v^{-\frac{1}{3}} \chi_{t^{\prime} \lesssim v^{-\frac{1}{3}}}\left(t^{\prime}\right)+v^{\frac{1}{3} \beta} t^{\prime 1-\beta} \chi_{t^{\prime} \approx v^{-\frac{1}{3}}}\left(t^{\prime}\right)\right) \\
& \times\langle\xi\rangle^{\sigma+1}\left|\hat{g}(\eta-\xi)_{<M / 8} \hat{\bar{h}}(\xi)_{M}\right| d \xi d \eta
\end{aligned}
$$




$$
\begin{aligned}
& \lesssim \sum_{M \geq 8}\left\|A^{\sigma} \bar{h} \sim M\right\|_{2}\left\|g_{M}\right\|_{H^{3}}\|\bar{h}\|_{H^{\sigma}}\left(v^{-\frac{1}{3}} \chi_{t^{\prime} \lesssim v^{-\frac{1}{3}}}\left(t^{\prime}\right)+v^{\frac{1}{3} \beta} t^{\prime 1-\beta} \chi_{t^{\prime} \approx v^{-\frac{1}{3}}}\left(t^{\prime}\right)\right) \\
& \lesssim\left\|A^{\sigma} \bar{h}\right\|_{2}\|g\|_{H^{3}}\|\bar{h}\|_{H^{\sigma}}\left(v^{-\frac{1}{3}} \chi_{t^{\prime} \lesssim v^{-\frac{1}{3}}}\left(t^{\prime}\right)+v^{\frac{1}{3} \beta} t^{\prime 1-\beta} \chi_{t^{\prime} \approx v^{-\frac{1}{3}}}\left(t^{\prime}\right)\right) .
\end{aligned}
$$

For the second term, we have $|\xi-\eta| \geq \frac{1}{10}|\eta| \approx|\xi|$, and thus we get

$$
\begin{aligned}
& \sum_{M \geq 8} \mid \int_{|\xi-\eta| \geq \frac{1}{10}|\eta|} A^{\sigma} \hat{\bar{h}}(\eta)\left[A^{\sigma}\left(\hat{g}(\eta-\xi)_{<M / 8} \widehat{\partial_{v} \bar{h}}(\xi)_{M}\right)\right. \\
& \left.\quad-\hat{g}(\eta-\xi)_{<M / 8} A^{\sigma} \widehat{\partial_{v} \bar{h}}(\xi)_{M}\right] d \xi d \eta \mid \\
& \quad \lesssim \sum_{M \geq 8} \int A^{\sigma} \hat{\bar{h}}(\eta)\langle\xi\rangle^{\sigma} \hat{g}(\xi)_{M} \widehat{\partial_{v} \bar{h}}(\eta-\xi)_{<M / 8} d \xi d \eta \\
& \quad \lesssim \sum_{M \geq 8}\left\|A^{\sigma} \bar{h}_{\sim M}\right\|_{2}\left\|g_{M}\right\|_{H^{\sigma}}\left\|\partial_{v} \bar{h}\right\|_{H^{1}} \lesssim\left\|A^{\sigma} \bar{h}\right\|_{2}\|g\|_{H^{\sigma}}\left\|\partial_{v} \bar{h}\right\|_{H^{1}}
\end{aligned}
$$

Now we turn to $V_{1, \sigma}^{\bar{h}, H H}$. We have

$$
|\eta| \lesssim|\eta-\xi|+|\xi| \approx|\xi| \approx|\eta-\xi|
$$

and then

$$
\begin{aligned}
\left|V_{1, \sigma}^{\bar{h}, H H}\right| & \lesssim \sum_{M \in \mathbb{D}}\left\|A^{\sigma} \bar{h}\right\|_{2}\left\|g_{M}\right\|_{H^{\sigma}}\|\bar{h} \sim M\|_{H^{3}} \\
& \lesssim\left\|A^{\sigma} \bar{h}\right\|_{2}\|g\|_{H^{\sigma}}\|\bar{h}\|_{H^{3}} .
\end{aligned}
$$

As suggested by Section $8.4, V_{2, \sigma}^{\bar{h}, \varepsilon}$ is not significantly harder than $V_{2, \sigma}^{\bar{h}}$. In fact the primary complications that arise in the treatment of $R_{N}^{\varepsilon, 1}$ do not arise in the treatment of $V_{2, \sigma}^{\bar{h}, \varepsilon}$. Hence we focus only on $V_{2, \sigma}^{\bar{h}}$; the control of $V_{2, \sigma}^{\bar{h}, \varepsilon}$ results in, at worst, similar contributions with an additional power of $\varepsilon$. Now we turn to $V_{2, \sigma}^{\bar{h}}$. We use the Littlewood-Paley decomposition in $v$ and get

$$
\begin{aligned}
V_{2, \sigma}^{\bar{h}}= & \frac{1}{t} \sum_{l \neq 0} \int \chi_{|\eta| \leq 100|l|} \frac{\langle\eta\rangle^{\sigma} \hat{\bar{h}}(t, \eta)}{w(t, \eta)} \frac{\langle\eta\rangle^{\sigma} \eta l}{w(t, \eta)} \overline{\hat{\phi}_{-l}(\eta-\xi) \hat{f}_{l}(\xi)} d \xi d \eta \\
& +\sum_{M \geq 8} \frac{1}{t} \sum_{l \neq 0} \int \chi_{|\eta| \geq 100|l|} \frac{\langle\eta\rangle^{\sigma}}{w(t, \eta)} \frac{\hat{\bar{h}}(t, \eta)}{w(t, \eta)} \frac{\langle\eta\rangle^{\sigma} \eta l}{\hat{\phi}_{-l}(\eta-\xi)_{<M / 8} \hat{f}_{l}(\xi)_{M}} d \xi d \eta \\
& +\sum_{M \geq 8} \frac{1}{t} \sum_{l \neq 0} \int \chi_{|\eta| \geq 100|l|} \frac{\langle\eta\rangle^{\sigma} \hat{\bar{h}}(t, \eta)}{w(t, \eta)} \frac{\langle\eta\rangle^{\sigma} \eta l}{w(t, \eta)} \frac{\hat{\phi}_{l}(\xi)_{M} \hat{f}_{-l}(\eta-\xi)_{<M / 8}}{w \xi} d \eta
\end{aligned}
$$




$$
\begin{aligned}
+\sum_{M \in \mathbb{D}} \sum_{\frac{1}{8}} \sum_{M \leq M^{\prime} \leq 8 M} \frac{1}{t} \sum_{l \neq 0} \int \chi_{|\eta| \geq 100|l|} \frac{\langle\eta\rangle^{\sigma} \hat{\bar{h}}(t, \eta)}{w(t, \eta)} \frac{\langle\eta\rangle^{\sigma} \eta l}{w(t, \eta)} \\
\times \overline{\hat{\phi}_{-l}(\eta-\xi)_{M^{\prime}} \hat{f}_{l}(\xi)_{M}} d \xi d \eta \\
=V_{2, \sigma}^{\bar{h}, z}+\sum_{M \geq 8} T_{M}^{\bar{h}}+\sum_{M \geq 8} R_{M}^{\bar{h}}+\mathcal{R}^{\bar{h}} .
\end{aligned}
$$

It is easy to obtain

$$
\left|V_{2, \sigma}^{\bar{h}, z}\right| \lesssim \frac{1}{t}\|\bar{h}\|_{H^{\sigma}}\left\|\phi_{\neq}\right\|_{H^{4}}\left\|f_{\neq}\right\|_{H^{\sigma}} \lesssim \frac{1}{t^{3}}\|\bar{h}\|_{H^{\sigma}}\left\|f_{\neq}\right\|_{H^{\sigma}}\left\|f_{\neq}\right\|_{H^{6}} .
$$

There is a loss of derivative in $T_{M}^{\bar{h}}$. By using the fact that

$$
|\eta|=|(\eta-\xi+l t)|+|(\xi-l t)| \lesssim\langle\eta-\xi+l t\rangle\langle\xi-l t\rangle,
$$

we get

$$
\begin{aligned}
\left|T_{M}^{\bar{h} \mid} \lesssim \frac{1}{t} \sum_{l \neq 0} \int 1_{|\eta| \geq 100|l|} \frac{\langle\eta\rangle^{\sigma}|\hat{\bar{h}}(t, \eta)|}{w(t, \eta)} \frac{\langle\xi\rangle^{\sigma}|l|}{w(t, \xi)}\right|\langle\eta-\xi+l t\rangle \hat{\phi}_{l}(\eta-\xi)_{<M / 8} \mid \\
\quad \times\left|\langle\xi-l t\rangle \hat{f}_{-l}(\xi)_{M}\right| d \xi d \eta \\
\lesssim \frac{1}{t}\left\|A^{\sigma} \bar{h}_{\sim M}\right\|_{2}\left\|\left(\sqrt{-\Delta_{L}} A^{\sigma} f_{\neq}\right)_{M}\right\|_{2}\left\|\partial_{z} \nabla_{L} \phi_{\neq}\right\|_{H^{2}} \\
\lesssim \frac{1}{t^{2}}\left\|A^{\sigma} \bar{h}_{\sim M}\right\|_{2}\left\|\left(\sqrt{-\Delta_{L}} A^{\sigma} f_{\neq}\right)_{M}\right\|_{2}\left\|f_{\neq}\right\|_{H^{3}} .
\end{aligned}
$$

For $R_{M}^{\bar{h}}$ we have

$$
\begin{aligned}
& \left|R_{M}^{\bar{h}}\right| \lesssim \frac{1}{t} \sum_{l \neq 0} \int 1_{|\eta| \geq 100|l|}\left|A^{\sigma} \hat{\bar{h}}(t, \eta)\right| A^{\sigma}(t, \xi) \frac{\xi / l^{2}}{\left\langle\frac{\xi}{l}-t\right\rangle^{2}} \widehat{\Delta_{L} \Delta_{t}^{-1} f_{l}}(\xi)_{M} \\
& \times{\widehat{\partial_{z} f}}_{-l}(\eta-\xi)_{<M / 8} d \xi d \eta \\
& \lesssim \frac{1}{t} \sum_{l \neq 0} \int 1_{|\eta| \geq 100|l|} \chi_{|l| \geq \frac{1}{10}} \sqrt{|\xi|}\left|A^{\sigma} \hat{\bar{h}}\right| A^{\sigma}(t, \xi) \frac{\xi / l^{2}}{\left\langle\frac{\xi}{l}-t\right\rangle^{2}} \widehat{\Delta_{L} \Delta_{t}^{-1} f_{l}}(\xi)_{M} \\
& \times{\widehat{\partial_{z} f}}_{-l}(\eta-\xi)_{<M / 8} d \xi d \eta \\
& +\frac{1}{t} \sum_{l \neq 0} \int 1_{|\eta| \geq 100|l|} 1_{|l| \leq \frac{1}{10} \sqrt{|\xi|}}\left[1_{t \in I_{l, \xi}}+1_{t \notin I_{l, \xi}}\right]\left|A^{\sigma} \hat{\bar{h}}\right|
\end{aligned}
$$

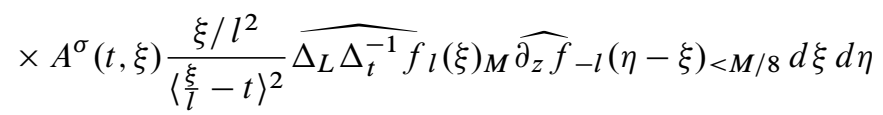

$$
\begin{aligned}
& =R_{M}^{\bar{h}, z}+R_{M, R}^{\bar{h}}+R_{M, N R}^{\bar{h}} .
\end{aligned}
$$


We have

$$
\left|R_{M}^{\bar{h}, z}\right| \lesssim \frac{1}{t}\left\|A^{\sigma} \bar{h}_{\sim M}\right\|_{2}\left\|A^{\sigma}\left(\Delta_{L} \Delta_{t}^{-1} f_{\neq}\right)_{M}\right\|_{2}\left\|f_{\neq}\right\|_{H^{3}}
$$

Next we treat $R_{M, N R}^{\bar{h}}$. We have

$$
\left|R_{M, N R}^{\bar{h}}\right| \lesssim \frac{1}{t}\left\|A^{\sigma} \bar{h}_{\sim M} t\right\|_{2}\left\|A^{\sigma}\left(\Delta_{L} \Delta_{t}^{-1} f_{\neq}\right)_{M}\right\|_{2}\left\|f_{\neq}\right\|_{H^{3}} .
$$

If $t \in I_{l, \xi}$ with $|l| \leq \frac{1}{10} \sqrt{|\xi|}$, then according to the integrand we get $|\xi-\eta| \leq \frac{3}{16}|\xi|$, and thus $t \in I_{k, \eta}$. By Lemma 3.2, we have the following three cases:

(a) $k=l$. We get

$$
\left|R_{M, R}^{\bar{h}}\right| \lesssim \frac{1}{t}\left\|\sqrt{\frac{\partial_{t} w}{w}} A^{\sigma} \bar{h} \sim M\right\|_{2}\left\|\sqrt{\frac{\partial_{t} w}{w}} \chi_{R} A^{\sigma}\left(\Delta_{L} \Delta_{t}^{-1} f_{\neq}\right)_{M}\right\|_{2}\left\|v^{-\frac{1}{3}}\left\langle v^{\frac{1}{3}} t\right\rangle^{1+\beta} f_{\neq}\right\|_{H^{6}} .
$$

(b) $\left|\frac{\eta}{k}-t\right| \gtrsim \frac{\eta}{k^{2}}$ and $\left|\frac{\xi}{k}-t\right| \gtrsim \frac{\xi}{k^{2}}$. Then the estimate is similar to $R_{M, N R}^{\bar{h}}$ and we get

$$
\left|R_{M, R}^{\bar{h}}\right| \lesssim \frac{1}{t}\left\|A^{\sigma} \bar{h}_{\sim M}\right\|_{2}\left\|A^{\sigma}\left(\Delta_{L} \Delta_{t}^{-1} f_{\neq}\right)_{M}\right\|_{2}\left\|f_{\neq}\right\|_{H^{3}} .
$$

(c) $|\eta-\xi| \gtrsim \frac{\xi}{l^{2}}$. Then we get

$$
\left|R_{M, R}^{\bar{h}}\right| \lesssim \frac{1}{t}\left\|A^{\sigma} \bar{h}_{\sim M} t\right\|_{2}\left\|A^{\sigma}\left(\Delta_{L} \Delta_{t}^{-1} f_{\neq}\right)_{M}\right\|_{2}\left\|f_{\neq}\right\|_{H^{4}} .
$$

The remainder term is easy to deal with. We use the fact that $|\eta| \lesssim|\eta-\xi|+|\xi|$ and $|\xi| \approx|\eta-\xi|$ and get

$$
\begin{aligned}
\left|\mathcal{R}^{\bar{h}}\right| \lesssim \sum_{M \in \mathbb{D}} \sum_{\frac{1}{8}} \sum_{M \leq M^{\prime} \leq 8 M} \frac{1}{t} \sum_{l \neq 0} \int \chi_{|\eta| \geq 100|l|}\left|A^{\sigma} \hat{\bar{h}}(t, \eta)\right|\langle\eta\rangle^{\sigma-2}\left|\hat{\phi}_{-l}(\eta-\xi)_{M^{\prime}}\right||\xi|^{3}|l| \\
\quad \times\left|\hat{f}_{l}(\xi)_{M}\right| d \xi d \eta \\
\lesssim \sum_{M \in \mathbb{D}} \frac{1}{t}\left\|A^{\sigma} \bar{h}\right\|_{2}\left\|P_{\neq} \phi \sim M\right\|_{H^{\sigma-2}}\left\|\left(f_{\neq}\right)_{M}\right\|_{H^{7}} \\
\lesssim \frac{1}{t}\left\|A^{\sigma} \bar{h}\right\|_{2}\left\|P_{\neq} \phi\right\|_{H^{\sigma-2}}\left\|f_{\neq}\right\|_{H^{7}} \lesssim \frac{1}{t}\left\|A^{\sigma} \bar{h}\right\|_{2}\left\|f_{\neq}\right\|_{H^{\sigma-2}}\left\|f_{\neq}\right\|_{H^{7}} .
\end{aligned}
$$

To treat the dissipation error term $V_{3, \sigma}^{\bar{h}}$, we have

$$
\begin{aligned}
\left|V_{3, \sigma}^{\bar{h}}\right| \lesssim & \int\langle\eta\rangle^{\sigma}|\hat{\bar{h}}(\eta)|\langle\eta\rangle^{\sigma}|G(\eta-\xi)||\xi|^{2}|\hat{\bar{h}}(\xi)| d \xi d \eta \\
\lesssim & \int_{|\eta-\xi| \leq|\xi|} 1_{|\eta| \leq 1|\hat{\bar{h}}(\eta)||G(\eta-\xi)||\xi|^{2}|\hat{\bar{h}}(\xi)| d \xi d \eta} \\
& +\int_{|\eta-\xi| \leq|\xi|} 1_{|\eta| \geq 1}\langle\eta\rangle^{\sigma}|\hat{\bar{h}}(\eta)|\langle\eta\rangle^{\sigma}|G(\eta-\xi)||\xi|^{2}|\hat{\bar{h}}(\xi)| d \xi d \eta \\
& +\int_{|\eta-\xi| \geq|\xi|} 1_{|\eta| \geq 1}\langle\eta\rangle^{\sigma}|\hat{\bar{h}}(\eta)|\langle\eta\rangle^{\sigma}|G(\eta-\xi)||\xi|^{2}|\hat{\bar{h}}(\xi)| d \xi d \eta \\
= & V_{3, \sigma}^{\bar{h},<1}+V_{3, \sigma}^{\bar{h}, L H}+V_{3, \sigma}^{\bar{h}, H L} .
\end{aligned}
$$


Now we treat $V_{3, \sigma}^{\bar{h},<1}$ :

$$
\left|V_{3, \sigma}^{\bar{h},<1}\right| \lesssim v\|\bar{h}\|_{L^{2}}\|\bar{h}\|_{H^{2}}\|G\|_{H^{2}} \lesssim v\|\bar{h}\|_{L^{2}}\|\bar{h}\|_{H^{2}}\|h\|_{H^{2}} .
$$

Next we turn to $V_{3, \sigma}^{\bar{h}, L H}$, in which case it holds that

$$
|\eta| \leq|\eta-\xi|+|\xi| \lesssim|\xi|
$$

Then we have

$$
\begin{aligned}
V_{3, \sigma}^{\bar{h}, L H} & \lesssim v \int_{|\eta-\xi| \leq|\xi|}|\eta|\langle\eta\rangle^{\sigma}|\hat{\bar{h}}(\eta)|\langle\xi\rangle^{\sigma-1}|G(\eta-\xi)||\xi|^{2}|\hat{\bar{h}}(\xi)| d \xi d \eta \\
& \lesssim v\|h\|_{H^{3}}\left\|\partial_{v} A^{\sigma} \bar{h}\right\|_{2}^{2} \lesssim \varepsilon v^{\frac{1}{3}} v\left\|\partial_{v} A^{\sigma} \bar{h}\right\|_{2}^{2} .
\end{aligned}
$$

Next we treat $V_{3, \sigma}^{\bar{h}, H L}$ and we get

$$
\begin{aligned}
\left|V_{3, \sigma}^{\bar{h}, H L}\right| & \lesssim v \int_{|\eta-\xi| \geq|\xi|}|\eta|\langle\eta\rangle^{\sigma}|\hat{\bar{h}}(\eta)|\langle\eta-\xi\rangle^{\sigma-1}|G(\eta-\xi)||\xi|^{2}|\hat{\bar{h}}(\xi)| d \xi d \eta \\
& \lesssim v\left\|\partial_{v} \bar{h}\right\|_{H^{\sigma}}\|h\|_{H^{\sigma-1}}\left\|\partial_{v} \bar{h}\right\|_{H^{3}} .
\end{aligned}
$$

Thus we conclude that

$$
\begin{aligned}
& \frac{1}{2} \frac{d}{d t}\left\|A^{\sigma} \bar{h}\right\|_{2}^{2}+\mathrm{CK}_{w}^{\bar{h}}+\frac{2}{t}\left\|A^{\sigma} \bar{h}\right\|_{2}^{2}+\frac{1}{2} v\left\|\partial_{v} A^{\sigma} \bar{h}\right\|_{2}^{2} \\
& \lesssim\left\|A^{\sigma} \bar{h}\right\|_{2}^{2}\|g\|_{H^{\sigma}}+\left\|A^{\sigma} \bar{h}\right\|_{2}^{2}\|g\|_{H^{3}}\left(v^{-\frac{1}{3}} \chi_{t^{\prime} \lesssim v^{-\frac{1}{3}}}\left(t^{\prime}\right)+v^{\frac{1}{3} \beta} t^{\prime 1-\beta} \chi_{t^{\prime} \gtrsim v^{-\frac{1}{3}}}\left(t^{\prime}\right)\right) \\
&+\frac{1}{t}\|\bar{h}\|_{H^{\sigma}}\left\|f_{\neq}\right\|_{H^{\sigma}}\left\|f_{\neq}\right\|_{H^{7}}+\frac{1}{t^{2}}\left\|A^{\sigma} \bar{h}\right\|_{2}\left\|\left(\sqrt{-\Delta_{L}} A^{\sigma} f_{\neq}\right)\right\|_{2}\left\|f_{\neq}\right\|_{H^{3}} \\
&+\frac{1}{t}\left\|A^{\sigma} \bar{h}\right\|_{2}\left\|A^{\sigma}\left(\Delta_{L} \Delta_{t}^{-1} f_{\neq}\right)\right\|_{2}\left\|f_{\neq}\right\|_{H^{3}} \\
&+\frac{1}{t}\left\|\sqrt{\frac{\partial_{t} w}{w}} A^{\sigma} \bar{h}\right\|_{2}\left\|\sqrt{\frac{\partial_{t} w}{w}} \chi_{R} A^{\sigma}\left(\Delta_{L} \Delta_{t}^{-1} f_{\neq}\right)\right\|_{2}\left\|v^{-\frac{1}{3}}\left\langle v^{\frac{1}{3}} t\right\rangle^{1+\beta} f_{\neq}\right\|_{H^{6}} \\
&+v\|\bar{h}\|_{L^{2}}\|\bar{h}\|_{H^{2}}\|h\|_{H^{2}},
\end{aligned}
$$

which gives by the bootstrap hypotheses that

$$
\begin{aligned}
&\left(t^{\frac{3}{2}}\left\|A^{\sigma} \bar{h}(t)\right\|_{2}\right)^{2}+\int_{1}^{t} t^{\prime 3} \mathrm{CK}_{w}^{\bar{h}} d t^{\prime}+\frac{1}{2} \int_{1}^{t} t^{\prime 2}\left\|A^{\sigma} \bar{h}\left(t^{\prime}\right)\right\|_{2}^{2} d t^{\prime} \\
& \quad+\frac{1}{2} v \int_{1}^{t} t^{\prime 3}\left\|\partial_{v} A^{\sigma} \bar{h}\left(t^{\prime}\right)\right\|_{2}^{2} d t^{\prime} \\
& \leq\left\|A^{\sigma} \bar{h}(1)\right\|_{2}^{2} \\
&+C\left[\|g\|_{L_{T}^{1}\left(H^{\sigma}\right)}+\int_{1}^{t} \frac{\varepsilon v^{\frac{1}{3}}}{t^{\prime 2}}\left(v^{-\frac{1}{3}} \chi_{t^{\prime} \lesssim v^{-\frac{1}{3}}}\left(t^{\prime}\right)+v^{\frac{1}{3} \beta} t^{\prime 1-\beta} \chi_{t^{\prime} \gtrsim v^{-\frac{1}{3}}}\left(t^{\prime}\right)\right) d t^{\prime}\right] \\
& \times\left\|t^{\frac{3}{2}}\right\| A^{\sigma} \bar{h}(t)\left\|_{2}\right\|_{L_{T}^{\infty}}^{2}
\end{aligned}
$$




$$
\begin{aligned}
& +C \int_{1}^{t} \frac{\varepsilon^{2} v^{\frac{2}{3}} t^{\frac{1}{2}}}{\left\langle v t^{\prime 3}\right\rangle} d t^{\prime}\left\|t^{\frac{3}{2}}\right\| A^{\sigma} \bar{h}(t)\left\|_{2}\right\|_{L_{T}^{\infty}} \\
& +C\left\|t^{\frac{3}{2}}\right\| A^{\sigma} \bar{h}(t)\left\|_{2}\right\|_{L_{T}^{\infty}}\left(\int_{1}^{t}\left[\frac{\varepsilon t^{\prime \frac{1}{2}}}{t^{\prime}} \frac{v^{-\frac{1}{6}}}{\left\langle v t^{\prime 3}\right\rangle}\right]^{2} d t^{\prime}\right)^{\frac{1}{2}} v^{\frac{1}{2}}\left\|\sqrt{-\Delta_{L}} f_{\neq}\right\|_{L_{T}^{2}\left(H^{\sigma}\right)} \\
& +C\left\|t^{\frac{3}{2}}\right\| A^{\sigma} \bar{h}(t)\left\|_{2}\right\|_{L_{T}^{\infty}}\left(\int_{1}^{t}\left[t^{\prime \frac{1}{2}} \frac{\varepsilon v^{-\frac{1}{6}}}{\langle t\rangle\left\langle v t^{3}\right\rangle}\right]^{2} d t^{\prime}\right)^{\frac{1}{2}} v^{\frac{1}{2}}\left\|\partial_{v} h\left(t^{\prime}\right)\right\|_{L_{T}^{2} H^{\sigma}} \\
& +C\left\|t^{\prime \frac{3}{2}} \sqrt{\frac{\partial_{t} w}{w}} A^{\sigma} \bar{h}\left(t^{\prime}\right)\right\|_{L_{T}^{2}\left(L^{2}\right)}\left\|\sqrt{\frac{\partial_{t} w}{w}} f_{\neq}\right\|_{L_{T}^{2}\left(H^{\sigma}\right)}\left\|\frac{\varepsilon^{2} t^{\frac{1}{2}}\left\langle v^{\frac{1}{3}} t\right\rangle^{1+\beta}}{\left\langle v t^{3}\right\rangle}\right\|_{L^{\infty}} \\
& +C\left\|t^{\prime \frac{3}{2}} \sqrt{\frac{\partial_{t} w}{w}} A^{\sigma} \bar{h}\left(t^{\prime}\right)\right\|_{L_{T}^{2}\left(L^{2}\right)}\left\|\frac{\varepsilon^{3} v^{\frac{1}{2}} t^{\frac{1}{2}}}{\left\langle v t^{3}\right\rangle}\right\|_{L_{T}^{2}} \\
& \leq\left\|A^{\sigma} \bar{h}(1)\right\|_{2}^{2}+\frac{1}{100}\left\|t^{\frac{3}{2}}\right\| A^{\sigma} \bar{h}(t)\left\|_{2}\right\|_{L_{T}^{\infty}}^{2}+\frac{1}{100} \int_{1}^{t} t^{\prime 3} \mathrm{CK}_{w}^{\bar{h}} d t^{\prime}+C \varepsilon^{4} v^{\frac{1}{3}} \text {. }
\end{aligned}
$$

The energy for $h$ in $H^{\sigma}$ is similar to the estimate of $H^{\sigma-1}$. We have

$$
\begin{aligned}
\frac{1}{2} \frac{d}{d t}\|h\|_{H^{\sigma}}^{2}= & -\int\left\langle\partial_{v}\right\rangle^{\sigma} h\left\langle\partial_{v}\right\rangle^{\sigma}\left(g \partial_{v} h-\bar{h}-v\left(v^{\prime}\right)^{2} \partial_{v}^{2} h\right) d v \\
= & -\int\left\langle\partial_{v}\right\rangle^{\sigma} h\left\langle\partial_{v}\right\rangle^{\sigma}\left(g \partial_{v} h\right) d v \\
& +\int\left\langle\partial_{v}\right\rangle^{\sigma} h\left\langle\partial_{v}\right\rangle^{\sigma} \bar{h} d v \\
& +v \int\left\langle\partial_{v}\right\rangle^{\sigma} h\left\langle\partial_{v}\right\rangle^{\sigma}\left(\left(\left(v^{\prime}\right)^{2}-1\right) \partial_{v}^{2} h\right) d v-v\left\|\partial_{v} h\right\|_{H^{\sigma}}^{2} \\
= & -v\left\|\partial_{v} h\right\|_{H^{\sigma}}^{2}+V_{1, \sigma}^{H, h}+V_{2, \sigma}^{H, h}+V_{3, \sigma}^{H, h}
\end{aligned}
$$

We get

$$
\left|V_{1, \sigma}^{H, h}\right| \lesssim\|g\|_{H^{\sigma}}\|h\|_{H^{\sigma}}^{2}, \quad\left|V_{2, \sigma}^{H, h}\right| \lesssim\|h\|_{H^{\sigma}}\|\bar{h}\|_{H^{\sigma}}, \quad\left|V_{3, \sigma}^{H, h}\right| \lesssim \varepsilon v^{\frac{1}{3}} v\left\|\partial_{v} h\right\|_{H^{\sigma}}^{2} .
$$

Thus we conclude that

$$
\begin{aligned}
\|h(t)\|_{H^{\sigma}}^{2}+v\left\|\partial_{v} h\right\|_{L_{T}^{2}\left(H^{\sigma}\right)}^{2} \leq & \|h(1)\|_{H^{\sigma}}^{2}+C\|g\|_{L_{T}^{1} H^{\sigma}}\|h\|_{L_{T}^{\infty} H^{\sigma}}^{2} \\
& +C\|h(1)\|_{H^{\sigma}}\|\bar{h}\|_{L_{T}^{1}\left(H^{\sigma}\right)} \\
\leq & \|h(1)\|_{H^{\sigma}}^{2}+\frac{1}{100}\|h\|_{L_{T}^{\infty} H^{\sigma}}^{2}+C \varepsilon^{3} v^{\frac{1}{3}}
\end{aligned}
$$

\subsection{Lower energy estimate}

9.3.1. Energy estimate of $\boldsymbol{g}$ in $\boldsymbol{H}^{\sigma-6}$. We have

$$
\begin{aligned}
\frac{d}{d t}\left(t^{4}\left\|\left\langle\partial_{v}\right\rangle^{\sigma-6} g\right\|_{2}^{2}\right) & =(4) t^{3}\left\|\left\langle\partial_{v}\right\rangle^{\sigma-6} g\right\|_{2}^{2}+t^{4} \frac{d}{d t}\left\|\left\langle\partial_{v}\right\rangle^{\sigma-6} g\right\|_{2}^{2} \\
& =(4) t^{3}\left\|\left\langle\partial_{v}\right\rangle^{\sigma-6} g\right\|_{2}^{2}+2 t^{4} \int\left\langle\partial_{v}\right\rangle^{\sigma-6} g\left\langle\partial_{v}\right\rangle^{\sigma-6} \partial_{t} g d v
\end{aligned}
$$




$$
\begin{aligned}
= & -2 t^{4} \int\left\langle\partial_{v}\right\rangle^{\sigma-6} g\left\langle\partial_{v}\right\rangle^{\sigma-6}\left(g \partial_{v} g\right) d v \\
& -2 t^{3} \int\left\langle\partial_{v}\right\rangle^{\sigma-6} g\left\langle\partial_{v}\right\rangle^{\sigma-6}\left(v^{\prime}\left\langle\nabla_{z, v}^{\perp} P_{\neq} \phi \cdot \nabla_{z, v} \tilde{u}\right\rangle\right) d v \\
& +2 t^{4} v \int\left\langle\partial_{v}\right\rangle^{\sigma-6} g\left\langle\partial_{v}\right\rangle^{\sigma-6}\left(\left(\left(v^{\prime}\right)^{2}-1\right) \partial_{v}^{2} g\right) d v-2 t^{4} v\left\|\partial_{v} g\right\|_{H^{\sigma-6}}^{2} \\
= & -2 t^{4} v\left\|\partial_{v} g\right\|_{H^{\sigma-6}}^{2}+V_{1}^{L, g}+V_{2}^{L, g}+V_{3}^{L, g} .
\end{aligned}
$$

As before we deal with $V_{1}^{L, g}$ by commutator estimate and integration by parts:

$$
\begin{aligned}
\left|V_{1}^{L, g}\right| & \left.\lesssim\left|t^{4} \int \partial_{v} g\right|\left\langle\partial_{v}\right\rangle^{\sigma-6} g\right|^{2} d v|+| t^{4} \int\left\langle\partial_{v}\right\rangle^{\sigma-6} g\left[\left\langle\partial_{v}\right\rangle^{\sigma-6}, g\right] \partial_{v} g d v \mid \\
& \lesssim t^{4}\|g\|_{H^{3}}\|g\|_{H^{\sigma-6}}^{2} .
\end{aligned}
$$

For $V_{2}^{L, g}$, by the fact that $\left\langle\nabla_{z, v}^{\perp} P_{\neq} \phi \cdot \nabla_{z, v} \tilde{u}\right\rangle=\partial_{v}\left\langle\partial_{z} P_{\neq} \phi \tilde{u}\right\rangle$, we get

$$
\begin{aligned}
\left|V_{2}^{L, g}\right| & \lesssim t^{3}\|g\|_{H^{\sigma-6}}\left(\left\|\left(\left(v^{\prime}-1\right)\left\langle\nabla_{z, v}^{\perp} P_{\neq} \phi \cdot \nabla_{z, v} \tilde{u}\right\rangle\right)\right\|_{H^{\sigma-6}}^{2}\right. \\
& \left.+\left\|\left\langle\nabla_{z, v}^{\perp} P_{\neq} \phi \cdot \nabla_{z, v} \tilde{u}\right\rangle\right\|_{H^{\sigma-6}}^{2}\right) \\
& \lesssim t^{3}\|g\|_{H^{\sigma-6}}\left(1+\|h\|_{H^{\sigma-6}}\right)\left\|\left\langle\partial_{z} P_{\neq} \phi \tilde{u}\right\rangle\right\|_{H^{\sigma-5}} \\
& \lesssim t^{3}\|g\|_{H^{\sigma-6}}\left(1+\|h\|_{H^{\sigma-6}}\right)\left\|P_{\neq} \phi\right\|_{H^{\sigma-4}}\|\tilde{u}\|_{H^{\sigma-5}} .
\end{aligned}
$$

Then by the bootstrap assumption and Lemma 4.1, we get

$$
\left|V_{2}^{L, g}\right| \lesssim\|g\|_{H^{\sigma-6}}\left\|f_{\neq}\right\|_{H^{\sigma-2}}\left\|f_{\neq}\right\|_{H^{\sigma-4}}
$$

For the dissipation error term we have

$$
\begin{aligned}
\left|V_{3}^{L, g}\right| \lesssim & \left|t^{4} v \int\left\langle\partial_{v}\right\rangle^{\sigma-6} g\left\langle\partial_{v}\right\rangle^{\sigma-6}\left(\left(\left(v^{\prime}\right)^{2}-1\right) \partial_{v}^{2} g\right) d v\right| \\
\lesssim & t^{4} v\left\|\left(\left(v^{\prime}\right)^{2}-1\right)\right\|_{H^{\sigma-6}}\left\|\partial_{v} g\right\|_{H^{\sigma-6}}^{2} \\
& +t^{4} v\left\|\partial_{v}\left(\left(v^{\prime}\right)^{2}-1\right)\right\|_{2}\left\|\partial_{v} g\right\|_{2}\|g\|_{H^{1}}+t^{4} v\left\|\left(\left(v^{\prime}\right)^{2}-1\right)\right\|_{2}\left\|\partial_{v} g\right\|_{2}^{2} \\
\lesssim & t^{4} v\left(1+\|h\|_{H^{2}}\right)\|h\|_{H^{\sigma-6}}\left\|\partial_{v} g\right\|_{H^{\sigma-6}}^{2} \\
& +t^{4} v\left(1+\|h\|_{H^{2}}\right)\left\|\partial_{v} h\right\|_{H^{2}}\left\|\partial_{v} g\right\|_{H^{2}}\|g\|_{H^{1}} .
\end{aligned}
$$

Thus, by the bootstrap assumption we get

$$
\begin{aligned}
\sup _{t \in[1, T]} t^{4}\|g(t)\|_{H^{\sigma-6}}^{2}+v & \int_{1}^{T} t^{\prime 4}\left\|\partial_{v} g\left(t^{\prime}\right)\right\|_{H^{\sigma-6}}^{2} d t^{\prime} \\
\leq\|g(1)\|_{H^{\sigma-6}}^{2}+C & \|g\|_{L_{T}^{1}\left(H^{\sigma-6}\right)} \sup _{t \in[1, T]} t^{4}\|g(t)\|_{H^{\sigma-6}}^{2} \\
& +\sup _{t^{\prime} \in[1, t]} t^{\prime 2}\left\|g\left(t^{\prime}\right)\right\|_{H^{\sigma-6}} \int_{1}^{T} \frac{\left\|f_{\neq}\right\|_{L_{T}^{\infty}\left(H^{\sigma-2}\right)}^{2}}{t^{2}} d t \\
& \left.+v\left\|\partial_{v} h\right\|_{L_{T}^{2}\left(H^{2}\right)}\left\|t^{2} \partial_{v} g\right\|_{L_{T}^{2}\left(H^{2}\right)} \sup _{t^{\prime} \in[1, t]} t^{\prime 2}\left\|g\left(t^{\prime}\right)\right\|_{H^{\sigma-6}}\right)
\end{aligned}
$$




$$
\leq\|g(1)\|_{H^{\sigma-6}}^{2}+C \varepsilon v^{\frac{1}{3}} \sup _{t \in[1, T]} t^{4}\|g(t)\|_{H^{\sigma-6}}^{2}+\varepsilon^{2} v^{\frac{2}{3}} \sup _{t^{\prime} \in[1, t]} t^{\prime 2}\left\|g\left(t^{\prime}\right)\right\|_{H^{\sigma-6}} .
$$

Thus, by taking $\varepsilon$ small enough we have proved (2.35).

9.3.2. Energy estimate of $\overline{\boldsymbol{h}}$ in $\boldsymbol{H}^{\boldsymbol{\sigma}-\boldsymbol{6}}$. The estimate is the same as the estimates of $g$ in lower Sobolev spaces. We have

$$
\begin{aligned}
\frac{d}{d t}\left(t^{4}\left\|\left\langle\partial_{v}\right\rangle^{\sigma-6} \bar{h}\right\|_{2}^{2}\right)= & 4 t^{3}\left\|\left\langle\partial_{v}\right\rangle^{\sigma-6} \bar{h}\right\|_{2}^{2}+t^{4} \frac{d}{d t}\left\|\left\langle\partial_{v}\right\rangle^{\sigma-6} \bar{h}\right\|_{2}^{2} \\
= & (4) t^{3}\left\|\left\langle\partial_{v}\right\rangle^{\sigma-6} \bar{h}\right\|_{2}^{2}+2 t^{4} \int\left\langle\partial_{v}\right\rangle^{\sigma-6} \bar{h}\left\langle\partial_{v}\right\rangle^{\sigma-6} \partial_{t} \bar{h} d v \\
= & -2 t^{4} \int\left\langle\partial_{v}\right\rangle^{\sigma-6} \bar{h}\left\langle\partial_{v}\right\rangle^{\sigma-6}\left(g \partial_{v} \bar{h}\right) d v \\
& -2 t^{3} \int\left\langle\partial_{v}\right\rangle^{\sigma-6} \bar{h}\left\langle\partial_{v}\right\rangle^{\sigma-6}\left(v^{\prime}\left\langle\nabla_{z, v}^{\perp} P_{\neq} \phi \cdot \nabla_{z, v} f\right\rangle\right) d v \\
& +2 t^{4} v \int\left\langle\partial_{v}\right\rangle^{\sigma-6} \bar{h}\left\langle\partial_{v}\right\rangle^{\sigma-6}\left(\left(\left(v^{\prime}\right)^{2}-1\right) \partial_{v}^{2} \bar{h}\right) d v-2 t^{4} v\left\|\partial_{v} \bar{h}\right\|_{H^{\sigma-6}}^{2} \\
= & -2 t^{4} v\left\|\partial_{v} \bar{h}\right\|_{H^{\sigma-6}}^{2}+V_{1}^{L, \bar{h}}+V_{2}^{L, \bar{h}}+V_{3}^{L, \bar{h}} .
\end{aligned}
$$

Then we have

$$
\left|V_{1}^{L, \bar{h}}\right| \lesssim\|g\|_{H^{\sigma-6}}\left\|t^{2} \bar{h}\right\|_{H^{\sigma-6}}^{2}
$$

For $V_{2}^{L, \bar{h}}$, we get by Lemma 4.1 that

$$
\begin{aligned}
\mid V_{2}^{L, \bar{h} \mid \lesssim} & t\left\|t^{2} \bar{h}\right\|_{H^{\sigma-6}}\left(1+\|h\|_{H^{\sigma-6}}\right)\left\|\left\langle\partial_{z} P_{\neq} \phi f_{\neq}\right\rangle\right\|_{H^{\sigma-5}} \\
\lesssim & t\left\|t^{2} \bar{h}\right\|_{H^{\sigma-6}}\left(1+\|h\|_{H^{\sigma-6}}\right)\left\|P_{\neq} \phi\right\|_{H^{\sigma-6}}\left\|f_{\neq}\right\|_{H^{2}} \\
& +t\left\|t^{2} \bar{h}\right\|_{H^{\sigma-6}}\left(1+\|h\|_{H^{\sigma-6}}\right)\left\|P_{\neq} \phi\right\|_{H^{3}}\left\|f_{\neq}\right\|_{H^{\sigma-5}} \\
\lesssim & t^{-1}\left\|t^{2} \bar{h}\right\|_{H^{\sigma-6}}\left(1+\|h\|_{H^{\sigma-6}}\right)\left\|P_{\neq} f\right\|_{H^{\sigma-4}}\left\|f_{\neq}\right\|_{H^{2}} \\
& +t^{-1}\left\|t^{2} \bar{h}\right\|_{H^{\sigma-6}}\left(1+\|h\|_{H^{\sigma-6}}\right)\left\|P_{\neq} f\right\|_{H^{5}}\left\|f_{\neq}\right\|_{H^{\sigma-5}} .
\end{aligned}
$$

Finally, for the dissipation error term we have

$$
\begin{aligned}
\left|V_{3}^{L, \bar{h}}\right| \lesssim & t^{4} v\left(1+\|h\|_{H^{2}}\right)\|h\|_{H^{\sigma-6}}\left\|\partial_{v} \bar{h}\right\|_{H^{\sigma-6}}^{2} \\
& +t^{4} v\left(1+\|h\|_{H^{2}}\right)\left\|\partial_{v} h\right\|_{H^{2}}\left\|\partial_{v} \bar{h}\right\|_{H^{2}}\|\bar{h}\|_{H^{1}}
\end{aligned}
$$

Thus, by the bootstrap hypotheses we obtain

$$
\begin{aligned}
\sup _{t \in[1, T]} & t^{4}\|\bar{h}(t)\|_{H^{\sigma-6}}^{2}+v \int_{1}^{T} t^{\prime 4}\left\|\partial_{v} \bar{h}\left(t^{\prime}\right)\right\|_{H^{\sigma-6}}^{2} d t^{\prime} \\
\leq & \|\bar{h}(1)\|_{H^{\sigma-6}}^{2}+C\left(\varepsilon v^{\frac{1}{3}} \sup _{t \in[1, T]} t^{4}\|\bar{h}(t)\|_{H^{\sigma-6}}^{2}+\varepsilon^{2} v^{\frac{1}{3}} \sup _{t \in[1, T]} t^{2}\|\bar{h}(t)\|_{H^{\sigma-6}}\right) \\
\leq & \|\bar{h}(1)\|_{H^{\sigma-6}}^{2}+\frac{1}{100} \sup _{t \in[1, T]} t^{4}\|\bar{h}(t)\|_{H^{\sigma-6}}^{2}+C \varepsilon^{4} v^{\frac{2}{3}} .
\end{aligned}
$$

Therefore, by taking $\varepsilon$ small enough we have proved (2.36). 


\section{Decay estimate of vorticity}

\subsection{Decay estimate of nonzero mode: enhanced dissipation}

Up to an adjustment of the constants in the bootstrap argument, it suffices to consider only $t$ such that $v t^{3} \gtrsim 1$ (say), as otherwise the decay estimate follows trivially from the higher regularity energy estimate.

Recall that

$$
\left\|A_{E}^{s} f\right\|_{2}^{2}=\sum_{k \neq 0} \int_{\eta}\langle k, \eta\rangle^{2 s}\left|D(t, \eta) \hat{f}_{k}(t, \eta)\right|^{2} d \eta
$$

Computing the time evolution of $\left\|A_{E}^{s} f\right\|_{2}$,

$$
\begin{aligned}
\frac{1}{2} \frac{d}{d t}\left\|A_{E}^{s} f\right\|_{2}^{2}= & \sum_{k \neq 0} \int_{\eta} \frac{\partial_{t} D(t, \eta)}{D(t, \eta)}\left|A_{E}^{s} \hat{f}_{k}(t, \eta)\right|^{2} d \eta \\
& -\int A_{E}^{s} f A_{E}^{s}(u \cdot \nabla f) d v d z+v \int A_{E}^{s} f A_{E}^{s}\left(\tilde{\Delta}_{t} f\right) d v d z \\
\leq & \frac{1}{8} v t^{2}\left\|1_{t \geq 2|\eta|} A_{E}^{s} \hat{f}_{k}(t, \eta)\right\|_{2}^{2} \\
& -\int A_{E}^{s} f A_{E}^{s}(u \cdot \nabla f) d v d z+v \int A_{E}^{s} f A_{E}^{s}\left(\tilde{\Delta}_{t} f\right) d v d z
\end{aligned}
$$

We write the dissipation term as

$$
\begin{aligned}
v \int A_{E}^{s} f A_{E}^{s}\left(\tilde{\Delta}_{t} f\right) d v d z= & -v\left\|\sqrt{-\Delta_{L}} A_{E}^{s} f\right\|_{2}^{2} \\
& -v \int A_{E}^{s} f A_{E}^{s}\left(\left(\left(v^{\prime}\right)^{2}-1\right)\left(\partial_{v}-t \partial_{z}\right)^{2} f\right) d v d z \\
= & -v\left\|\sqrt{-\Delta_{L}} A_{E}^{s} f\right\|_{2}^{2}+E^{v}
\end{aligned}
$$

First, we need to cancel the growing term cause by $D(t, \eta)$. Indeed, we have

$$
\begin{aligned}
\frac{1}{8} v t^{2} & \left\|1_{t \geq 2|\eta|} A_{E}^{s} \hat{f}_{k}(t, \eta)\right\|_{2}^{2}-v\left\|\sqrt{-\Delta_{L}} A_{E}^{s} f\right\|_{2}^{2} \\
& =\sum_{k \neq 0} \int v\left(\frac{1}{8} t^{2} 1_{t \geq 2 \eta}-k^{2}-(\eta-k t)^{2}\right)\left|A_{E}^{s} \hat{f}_{k}(\eta)\right|^{2} d \eta \\
& \leq-\frac{1}{8} v\left\|\sqrt{-\Delta_{L}} A_{E}^{s} f\right\|_{2}^{2},
\end{aligned}
$$

which gives that

$$
\frac{1}{2} \frac{d}{d t}\left\|A_{E}^{s} f\right\|_{2}^{2} \leq-\int A_{E}^{s} f A_{E}^{s}(u \cdot \nabla f) d v d z-\frac{1}{8} v\left\|\sqrt{-\Delta_{L}} A_{E}^{s} f\right\|_{2}^{2}+E^{v} .
$$


10.1.1. Euler nonlinearity. We first divide into zero and nonzero frequency contributions, as they will be treated differently:

$$
\begin{aligned}
-\int A_{E}^{s} f A_{E}^{s}(u \cdot \nabla f) d v d z= & -\int A_{E}^{s} f A_{E}^{s}\left(g \partial_{v} f\right) d v d z \\
& -\int A_{E}^{s} f A_{E}^{s}\left(v^{\prime} \nabla^{\perp} P_{\neq} \phi \cdot \nabla f\right) d v d z \\
= & E_{1}+E_{2} .
\end{aligned}
$$

For $E_{1}$ we use the commutator trick and the paraproduct (in both $z$ and $v$ ),

$$
\begin{aligned}
E_{1} & =\frac{1}{2} \int \partial_{v} g\left|A_{E}^{s} f\right|^{2} d v d z+\int A_{E}^{s} f\left[g \partial_{v} A_{E}^{s} f-A_{E}^{s}\left(g \partial_{v} f\right)\right] d v d z \\
& =\frac{1}{2} \int \partial_{v} g\left|A_{E}^{s} f\right|^{2} d v d z+\sum_{N \geq 8} T_{N}^{0}+\sum_{N \geq 8} R_{N}^{0}+\mathcal{R}^{0}
\end{aligned}
$$

where

$$
\begin{aligned}
T_{N}^{0} & =\int A_{E}^{s} f\left[g_{<N / 8} \partial_{v} A_{E}^{s} f_{N}-A_{E}^{s}\left(g_{<N / 8} \partial_{v} f_{N}\right)\right] d v d z \\
R_{N}^{0} & =\int A_{E}^{s} f\left[g_{N} \partial_{v} A_{E}^{s} f_{<N / 8}-A_{E}^{s}\left(g_{N} \partial_{v} f_{<N / 8}\right)\right] d v d z \\
\mathcal{R}^{0} & =\sum_{N \in \mathbb{D}} \sum_{N / 8 \leq N^{\prime} \leq 8 N} \int A_{E}^{s} f\left[g_{N^{\prime}} \partial_{v} A_{E}^{s} f_{N}-A_{E}^{s}\left(g_{N^{\prime}} \partial_{v} f_{N}\right)\right] d v d z
\end{aligned}
$$

Treatment of $T_{N}^{0}$. We get

$$
\begin{aligned}
T_{N}^{0}= & -i \sum_{k \neq 0} \int_{\eta, \xi} A_{E}^{s} \overline{\hat{f}}_{k}(\eta) D(\eta)\left(\langle k, \eta\rangle^{s}-\langle k, \xi\rangle^{s}\right) \hat{g}(\eta-\xi)_{<N / 8} \xi \hat{f}_{k}(\xi)_{N} d \eta d \xi \\
& -i \sum_{k \neq 0} \int_{\eta, \xi} A_{E}^{s} \overline{\hat{f}}_{k}(\eta)\langle k, \xi\rangle^{s}(D(\eta)-D(\xi)) \hat{g}(\eta-\xi)_{<N / 8} \xi \hat{f}_{k}(\xi)_{N} d \eta d \xi \\
= & T_{N}^{0,1}+T_{N}^{0,2} .
\end{aligned}
$$

For the term $T_{N}^{0,1}$, by Lemma 3.4 and the fact that $|k, \eta| \approx|k, \xi|$ and

$$
\left|\langle k, \eta\rangle^{s}-\langle k, \xi\rangle^{s}\right| \lesssim \frac{|\xi-\eta|}{\langle\eta\rangle+\langle\xi\rangle}\langle k, \xi\rangle^{s},
$$

we have

$$
\begin{aligned}
\left|T_{N}^{0,1}\right| & \lesssim \sum_{k \neq 0} \int_{\eta, \xi}\left|A_{E}^{s} \overline{\hat{f}}_{k}(\eta)\right|\langle\eta-\xi\rangle^{4}\left|\hat{g}(\eta-\xi)_{<N / 8}\right| \frac{|\xi|}{\langle\xi\rangle} A_{E}^{s} \hat{f}_{k}(\xi)_{N} d \eta d \xi \\
& \lesssim\left\|A_{E}^{S} f_{\sim N}\right\|_{2}\left\|A_{E}^{s} f_{N}\right\|_{2}\|g\|_{H^{5}} .
\end{aligned}
$$


We turn to $T_{N}^{0,2}$. By Lemma 3.4 we get

$$
\begin{aligned}
\left|T_{N}^{0,2}\right| & \lesssim \sum_{k \neq 0} \int_{\eta, \xi}\left|A_{E}^{s} \overline{\hat{f}}_{k}(\eta)\right|\langle\eta-\xi\rangle^{3}\left|\hat{g}(\eta-\xi)_{<N / 8}\right| \frac{|\xi|}{\langle\xi\rangle} A_{E}^{s} \hat{f}_{k}(\xi)_{N} d \eta d \xi \\
& \lesssim\left\|A_{E}^{s} f_{\sim N}\right\|_{2}\left\|A_{E}^{s} f_{N}\right\|_{2}\|g\|_{H^{5}} .
\end{aligned}
$$

Thus we get by (A.1) and (A.2) that

$$
\sum_{N \geq 8}\left|T_{N}^{0}\right| \leq\|g\|_{H^{5}}\left\|A_{E}^{s} f\right\|_{2}^{2}
$$

Treatment of $\boldsymbol{R}_{N}^{\mathbf{0}}$. The "reaction" term $R_{N}^{0}$ is dealt with easily by "moving" the derivative to $g$. We have

$$
\left|\int A_{E}^{s} f g_{N} \partial_{v} A_{E}^{s} f_{<N / 8} d v d z\right| \lesssim\left\|A_{E}^{s} f_{\sim N}\right\|_{2}\left\|g_{N}\right\|_{H^{2}}\left\|A_{E}^{s} f\right\|_{2},
$$

and by Lemma 3.4 and the fact that $A_{E}^{s}\left(g_{N} \partial_{v}(f)_{<N / 8}\right)=A_{E}^{s}\left(g_{N} \partial_{v}\left(f_{\neq}\right)_{<N / 8}\right)$, we get

$$
\begin{aligned}
& \left|\int A_{E}^{s} f A_{E}^{s}\left(g_{N} \partial_{v}\left(f_{\neq}\right)_{<N / 8}\right) d v d z\right| \\
& \quad \lesssim\left|\sum_{k} \int_{\eta, \xi} A_{E}^{s}\right| \hat{f}_{k}(\eta)\left|\langle\eta-\xi\rangle^{3}\langle k, \eta\rangle^{s}\right| \hat{g}_{N}(\xi-\eta)|| \xi|| \hat{f}_{k}(\xi)_{<N / 8}|d \eta d \xi| .
\end{aligned}
$$

On the support of the integrand we have $|k, \eta| \approx|\xi-\eta| \gtrsim|k, \xi|$, and thus

$$
\left|\int A_{E}^{s} f A_{E}^{s}\left(g_{N} \partial_{v}\left(f_{\neq}\right)_{<N / 8}\right) d v d z\right| \lesssim\left\|A_{E}^{s} f_{\sim N}\right\|_{2}\left\|A_{E}^{s} f\right\|_{2}\left\|g_{N}\right\|_{H^{s+4}} .
$$

The treatment of the remainder terms is similar to the reaction term. We have

$$
\begin{aligned}
\mathcal{R}^{0} & \lesssim \sum_{N \in \mathbb{D}}\left\|A_{E}^{s} f\right\|_{2}\left\|A_{E}^{s} f_{N}\right\|_{2}\left\|g_{\sim N}\right\|_{H^{s+4}} \\
& \lesssim\|g\|_{H^{s+4}}\left\|A_{E}^{s} f\right\|_{2}^{2} .
\end{aligned}
$$

Therefore, by the bootstrap hypotheses we conclude that

$$
\left|E_{1}\right| \lesssim\|g\|_{H^{s+4}}\left\|A_{E}^{s} f\right\|_{2}^{2} \leq \frac{\varepsilon v^{\frac{1}{3}}}{\langle t\rangle^{2}}\left\|A_{E}^{s} f\right\|_{2}^{2} .
$$

Treatment of $\boldsymbol{E}_{\mathbf{2}}$. Next we turn to $E_{2}$. Now we need to use the inviscid damping to obtain decay in time. Roughly speaking, if $f$ is of zero mode, we will land the operator $A_{E}^{s}$ on $P_{\neq} \phi$ and use the lossy elliptic estimate for $A_{E}^{S}$.

Thus we get by Lemma 3.5 that

$$
\begin{aligned}
\left|E_{2}\right| \lesssim & \left\|A_{E}^{s} f\right\|_{2}\left\|A_{E}^{s}\left(\left(v^{\prime}-1\right) \nabla^{\perp} P_{\neq} \phi \cdot \nabla f\right)\right\|_{2}+\left\|A_{E}^{s} f\right\|_{2}\left\|A_{E}^{s}\left(\nabla^{\perp} P_{\neq} \phi \cdot \nabla f\right)\right\|_{2} \\
& \lesssim\left\|A_{E}^{s} f\right\|_{2}\left(1+\|h\|_{H^{s+3}}\right)\left\|A_{E}^{s}\left(\nabla^{\perp} P_{\neq} \phi \cdot \nabla f_{\neq}\right)\right\|_{2} \\
& \lesssim\left\|A_{E}^{s} f\right\|_{2}\left(1+\|h\|_{H^{s+3}}\right)\left\|A_{E}^{s} P_{\neq} \phi\right\|_{2}\|f\|_{H^{s+5}} \\
& +\left\|A_{E}^{s} f\right\|_{2}^{2}\left(1+\|h\|_{H^{s+3}}\right)\left\|P_{\neq} \phi\right\|_{H^{s+5}} .
\end{aligned}
$$


Thus, by the bootstrap hypotheses and Lemma 4.4 we have

$$
\left|E_{2}\right| \lesssim \frac{\varepsilon v^{\frac{1}{3}}}{\langle t\rangle^{2}}\left(\left\|A_{E}^{s} f\right\|_{2}^{2}+\left\|A^{\sigma} f\right\|_{2}\left\|A_{E}^{S} f\right\|_{2}\right) .
$$

10.1.2. Dissipation error term. By Lemma 3.4 and the fact that

$$
|\xi-k t| \leq|\xi-\eta|+|\eta-k t| \leq\langle\xi-\eta\rangle \sqrt{k^{2}+|\eta-k t|^{2}},
$$

we have

$$
\begin{aligned}
& \left.\left|E^{v}\right| \lesssim v \sum_{k \neq 0} \int_{\eta, \xi} \mid A_{E}^{s} \hat{f}_{k}(\eta) A_{E}^{s}(k, \eta) \widehat{\left(1-\left(v^{\prime}\right)^{2}\right.}\right)(\eta-\xi)|\xi-k t|^{2} \hat{f}_{k}(\xi) \mid d \eta d \xi \\
& \left.\lesssim v \sum_{k \neq 0} \int_{\eta, \xi} \mid A_{E}^{s} \sqrt{k^{2}+|\eta-k t|^{2}} \hat{f}_{k}(\eta) A_{E}^{s}(k, \eta)\langle\xi-\eta\rangle \overline{\left(1-\left(v^{\prime}\right)^{2}\right.}\right)(\eta-\xi) \\
& \times|\xi-k t| \hat{f}_{k}(\xi) \mid d \eta d \xi \\
& \left.\lesssim v \sum_{k \neq 0} \int_{\eta, \xi} \mid A_{E}^{s} \sqrt{k^{2}+|\eta-k t|^{2}} \hat{f}_{k}(\eta)\langle\xi-\eta\rangle^{4} \widehat{\left(1-\left(v^{\prime}\right)^{2}\right.}\right)(\eta-\xi)|\xi-k t| \\
& \times A_{E}^{s}(k, \xi) \hat{f}_{k}(\xi) \mid d \eta d \xi \\
& \lesssim v\left\|\sqrt{-\Delta_{L}} A_{E}^{s} f\right\|_{2}^{2}\left\|\left(1-\left(v^{\prime}\right)^{2}\right)\right\|_{H^{6}} \lesssim v\left(1+\|h\|_{H^{2}}\right)\|h\|_{H^{6}}\left\|\sqrt{-\Delta_{L}} A_{E}^{s} f\right\|_{2}^{2} \text {. }
\end{aligned}
$$

Thus we get

$$
\begin{aligned}
\frac{1}{2} \frac{d}{d t}\left\|A_{E}^{s} f\right\|_{2}^{2} \leq & E_{1}+E_{2}-\frac{1}{8} v\left\|\sqrt{-\Delta_{L}} A_{E}^{s} f\right\|_{2}^{2}+E^{v} \\
\leq & \frac{C \varepsilon v^{\frac{1}{3}}}{\langle t\rangle^{2}}\left\|A_{E}^{s} f\right\|_{2}^{2}+\frac{C \varepsilon v^{\frac{1}{3}}}{\langle t\rangle^{2}}\left\|A^{\sigma} f\right\|_{2}^{2} \\
& -\frac{1}{8} v\left\|\sqrt{-\Delta_{L}} A_{E}^{s} f\right\|_{2}^{2}+C v \varepsilon v^{\frac{1}{3}}\left\|\sqrt{-\Delta_{L}} A_{E}^{s} f\right\|_{2}^{2},
\end{aligned}
$$

which gives that

$$
\begin{aligned}
& \left\|A_{E}^{s} f(t)\right\|_{2}^{2}+\int_{1}^{t} \frac{1}{5} v\left\|\sqrt{-\Delta_{L}} A_{E}^{s} f\left(t^{\prime}\right)\right\|_{2}^{2} d t^{\prime} \\
& \quad \leq\left\|A_{E}^{s} f(1)\right\|_{2}^{2}+C \varepsilon v^{\frac{1}{3}}\left\|A_{E}^{s} f(t)\right\|_{2}^{2}+C \varepsilon^{3} v .
\end{aligned}
$$

Thus, by taking $\varepsilon$ small enough we have proved (2.34).

\subsection{Decay of the zero mode}

Here we start the proof of (2.37). The zero mode $f_{0}$ satisfies

$$
\partial_{t} f_{0}+g \partial_{v} f_{0}+v^{\prime}\left\langle\nabla_{z, v}^{\perp} P_{\neq} \phi \cdot \nabla_{z, v} f\right\rangle-v\left(v^{\prime}\right)^{2} \partial_{v}^{2} f_{0}=0 .
$$


We want to prove that the zero mode slightly decays. It is natural to study the time evolution of

$$
\varepsilon_{L, 0}(t)=\left\|\left\langle\partial_{v}\right\rangle^{s} f_{0}\right\|_{2}^{2}+\frac{t v}{2}\left\|\left\langle\partial_{v}\right\rangle^{s} \partial_{v} f_{0}\right\|_{2}^{2}
$$

We get

$$
\begin{aligned}
\frac{d}{d t} \mathcal{E}_{L, 0}(t)= & \frac{1}{2} v\left\|\left\langle\partial_{v}\right\rangle^{s} \partial_{v} f_{0}\right\|_{2}^{2}+t v \frac{1}{2} \frac{d}{d t}\left(\left\|\left\langle\partial_{v}\right\rangle^{s} \partial_{v} f_{0}\right\|_{2}^{2}\right)+\frac{d}{d t}\left(\left\|\left\langle\partial_{v}\right\rangle^{s} f_{0}\right\|_{2}^{2}\right) \\
= & -\frac{3}{2} v\left\|\left\langle\partial_{v}\right\rangle^{s} \partial_{v} f_{0}\right\|_{2}^{2}-v^{2} t\left\|\partial_{v}^{2} f_{0}\right\|_{H^{s}}^{2} \\
& -v t \int\left\langle\partial_{v}\right\rangle^{s} \partial_{v} f_{0}\left\langle\partial_{v}\right\rangle^{s} \partial_{v}\left(g \partial_{v} f_{0}\right) d v-2 \int\left\langle\partial_{v}\right\rangle^{s} f_{0}\left\langle\partial_{v}\right\rangle^{s}\left(g \partial_{v} f_{0}\right) d v \\
& -v t \int\left\langle\partial_{v}\right\rangle^{s} \partial_{v} f_{0}\left\langle\partial_{v}\right\rangle^{s} \partial_{v}\left(v^{\prime}\left\langle\nabla_{z, v}^{\perp} P_{\neq} \phi \cdot \nabla_{z, v} f\right\rangle\right) d v \\
& -2 \int\left\langle\partial_{v}\right\rangle^{s} f_{0}\left\langle\partial_{v}\right\rangle^{s}\left(v^{\prime}\left\langle\nabla_{z, v}^{\perp} P_{\neq} \phi \cdot \nabla_{z, v} f\right\rangle\right) d v \\
& +v^{2} t \int\left\langle\partial_{v}\right\rangle^{s} \partial_{v} f_{0}\left\langle\partial_{v}\right\rangle^{s} \partial_{v}\left(\left(\left(v^{\prime}\right)^{2}-1\right) \partial_{v}^{2} f_{0}\right) d v \\
& +2 v \int\left\langle\partial_{v}\right\rangle^{s} f_{0}\left\langle\partial_{v}\right\rangle^{s}\left(\left(\left(v^{\prime}\right)^{2}-1\right) \partial_{v}^{2} f_{0}\right) d v \\
= & -\frac{3}{2} v\left\|\left\langle\partial_{v}\right\rangle^{s} \partial_{v} f_{0}\right\|_{2}^{2}-v^{2} t\left\|\partial_{v}^{2} f_{0}\right\|_{H^{s}}^{2} \\
& +V_{1,1}+V_{1,2}+V_{2,1}+V_{2,2}+V_{3,1}+V_{3,2} .
\end{aligned}
$$

To treat $V_{1,1}$ and $V_{1,2}$, we use the commutator estimate and integration by parts:

$$
\begin{aligned}
\left|V_{1,1}\right|+\left|V_{1,2}\right| \lesssim & \left\|\partial_{v} g\right\|_{L^{\infty}}\left(\left\|f_{0}\right\|_{H^{s}}^{2}+v t\left\|\partial_{v} f_{0}\right\|_{H^{s}}^{2}\right) \\
& +\left\|f_{0}\right\|_{H^{s}}\left\|\left[\left\langle\partial_{v}\right\rangle^{s}, g\right] \partial_{v} f_{0}\right\|_{L^{2}}+v t\left\|\partial_{v} f_{0}\right\|_{H^{s}}\left\|\left[\left\langle\partial_{v}\right\rangle^{s} \partial_{v}, g\right] \partial_{v} f_{0}\right\|_{L^{2}} \\
\lesssim & \|g\|_{H^{s}}\left(\left\|f_{0}\right\|_{H^{s}}^{2}+\frac{1}{2} v t\left\|\partial_{v} f_{0}\right\|_{H^{s}}^{2}\right) .
\end{aligned}
$$

Next we turn to $V_{2,1}, V_{2,2}$ : by using the fact that

$$
\left\langle\nabla_{z, v}^{\perp} P_{\neq} \phi \cdot \nabla_{z, v} f\right\rangle=\partial_{v}\left\langle\partial_{z} P_{\neq} \phi f_{\neq}\right\rangle,
$$

we get

$$
\begin{aligned}
\left|V_{2,1}\right| & \lesssim v t\left\|\partial_{v} f_{0}\right\|_{H^{s}}\left(\left\|h \partial_{v}\left\langle\partial_{z} P_{\neq} \phi f_{\neq}\right\rangle\right\|_{H^{s+1}}+\left\|\partial_{v}\left\langle\partial_{z} P_{\neq} \phi f_{\neq}\right\rangle\right\|_{H^{s+1}}\right) \\
& \lesssim v t\left(1+\|h\|_{H^{s+1}}\right)\left\|\partial_{v} f_{0}\right\|_{H^{s}}\left\|P_{\neq} \phi\right\|_{H^{s+3}}\left\|f_{\neq}\right\|_{H^{s+2}}
\end{aligned}
$$

and similarly

$$
\begin{aligned}
\left|V_{2,2}\right| & \lesssim\left\|f_{0}\right\|_{H^{s}}\left(\left\|h \partial_{v}\left\langle\partial_{z} P_{\neq} \phi f_{\neq}\right\rangle\right\|_{H^{s}}+\left\|\partial_{v}\left\langle\partial_{z} P_{\neq} \phi f_{\neq}\right\rangle\right\|_{H^{s+1}}\right) \\
& \lesssim\left(1+\|h\|_{H^{s}}\right)\left\|f_{0}\right\|_{H^{s}}\left\|P_{\neq} \phi\right\|_{H^{s+2}}\left\|f_{\neq}\right\|_{H^{s+1}}
\end{aligned}
$$


Finally, we turn to $V_{3,1}, V_{3,2}$ : as before we have

$$
\left|V_{3,1}\right| \lesssim v^{2} t\left\|\partial_{v}^{2} f_{0}\right\|_{H^{s}}^{2}\left\|\left(v^{\prime}\right)^{2}-1\right\|_{H^{s+1}}+v^{2} t\left\|\partial_{v}\left(\left(v^{\prime}\right)^{2}-1\right)\right\|_{2}\left\|\partial_{v}^{2} f_{0}\right\|_{2}\left\|\partial_{v} f_{0}\right\|_{H^{1}}
$$

and

$$
\left|V_{3,2}\right| \lesssim v\left\|\partial_{v}^{2} f_{0}\right\|_{H^{s}}^{2}\left\|\left(v^{\prime}\right)^{2}-1\right\|_{H^{s}}+v\left\|\partial_{v}\left(\left(v^{\prime}\right)^{2}-1\right)\right\|_{2}\left\|\partial_{v} f_{0}\right\|_{2}\left\|f_{0}\right\|_{H^{1}} .
$$

Thus, by the bootstrap assumption we get

$$
\begin{aligned}
\sup _{t^{\prime} \in[1, t]} \mathcal{E}_{L, 0}\left(t^{\prime}\right)+v \int_{1}^{T}\left\|\partial_{v} f_{0}(t)\right\|_{H^{s}}^{2} d t \\
\leq\left(\left\|\left\langle\partial_{v}\right\rangle^{s} f_{0}(1)\right\|_{2}^{2}+\frac{v}{2}\left\|\left\langle\partial_{v}\right\rangle^{s} \partial_{v} f_{0}(1)\right\|_{2}^{2}\right) \\
+C\left[\|g\|_{L_{T}^{1}\left(H^{s}\right)} \sup _{t^{\prime} \in[1, t]} \varepsilon_{L, 0}\left(t^{\prime}\right)\right. \\
\quad+\left[\sup _{t^{\prime} \in[1, t]} \varepsilon_{L, 0}\left(t^{\prime}\right)\right]^{\frac{1}{2}}\left\|f_{\neq}\right\|_{L_{T}^{\infty}\left(H^{s+5}\right)}^{2} \int_{1}^{T} \frac{\sqrt{v t}+1}{t^{2}} d t \\
\quad+v\left\|\partial_{v} h\right\|_{L_{T}^{2} H^{1}}\left\|\sqrt{v t} \partial_{v}^{2} f_{0}\right\|_{L_{T}^{2}\left(L^{2}\right)}\left\|\sqrt{v t} \partial_{v} f_{0}\right\|_{L_{T}^{\infty}\left(H^{1}\right)} \\
\quad+v\left\|\partial_{v} h\right\|_{L_{T}^{2}\left(L^{2}\right)}\left\|\partial_{v} f_{0}\right\|_{\left.L_{T}^{2}\left(L^{2}\right)\left\|f_{0}\right\|_{L_{T}^{\infty}\left(H^{1}\right)}\right]} \\
\leq\left(\left\|\left\langle\partial_{v}\right\rangle^{s} f_{0}(1)\right\|_{2}^{2}+\frac{v}{2}\left\|\left\langle\partial_{v}\right\rangle^{s} \partial_{v} f_{0}(1)\right\|_{2}^{2}\right)+C \varepsilon v^{\frac{1}{3}} \sup _{t^{\prime} \in[1, t]} \mathcal{E}_{L, 0}\left(t^{\prime}\right) \\
\quad+C \varepsilon^{2} v^{\frac{2}{3}}\left[\sup _{t^{\prime} \in[1, t]} \varepsilon_{L, 0}\left(t^{\prime}\right)\right]^{\frac{1}{2}}+C \varepsilon^{3} v \\
\leq\left(\left\|\left\langle\partial_{v}\right\rangle^{s} f_{0}(1)\right\|_{2}^{2}+\frac{v}{2}\left\|\left\langle\partial_{v}\right\rangle^{s} \partial_{v} f_{0}(1)\right\|_{2}^{2}\right)+C \varepsilon \sup _{t^{\prime} \in[1, t]} \varepsilon_{L, 0}\left(t^{\prime}\right)+C \varepsilon^{3} v .
\end{aligned}
$$

Thus, by taking $\varepsilon$ small enough we have proved (2.37).

\section{A. Functional analysis tools}

\section{A.1. Littlewood-Paley decomposition and paraproducts}

In this section we fix conventions and notation regarding Fourier analysis, LittlewoodPaley and paraproduct decompositions. See e.g. [1,11] for more details.

For $f(z, v)$ in the Schwartz space, we define the Fourier transform $\hat{f}_{k}(\eta)$, where $(k, \eta) \in \mathbb{Z} \times \mathbf{R}$, as

$$
\hat{f}_{k}(\eta)=\frac{1}{2 \pi} \int_{\mathbb{T} \times R} f(z, v) e^{-i k z-i v \eta} d z d v
$$

and the Fourier inversion formula is

$$
f(z, v)=\frac{1}{2 \pi} \sum_{k \in \mathbb{Z}} \int_{\mathbf{R}} \hat{f}_{k}(\eta) e^{i k z+i v \eta} d \eta .
$$


With these definitions we have

$$
\begin{gathered}
\int f(z, v) g(z, v) d z d v=\sum_{k} \int \hat{f}_{k}(\eta) \hat{g}_{k}(\eta) d \eta, \\
\hat{f} g=\hat{f} * \hat{g} .
\end{gathered}
$$

This work makes heavy use of the Littlewood-Paley dyadic decomposition. Here we fix conventions and review the basic properties of this classical theory; see e.g. [1] for more details. First, we define the Littlewood-Paley decomposition only in the $v$ variable. Let $\psi \in C_{0}^{\infty}(\mathbf{R} ; \mathbf{R})$ be such that $\psi(\xi)=1$ for $|\xi| \leq \frac{1}{2}$ and $\psi(\xi)=0$ for $|\xi| \geq \frac{3}{4}$, and define $\chi(\xi)=\psi\left(\frac{\xi}{2}\right)-\psi(\xi)$ supported in the range $\xi \in\left(\frac{1}{2}, \frac{3}{2}\right)$. Then we have the partition of unity

$$
1=\psi(\xi)+\sum_{M \in 2^{\mathbb{N}}} \chi_{M}(\xi),
$$

where we mean that the sum runs over the dyadic numbers $M=1,2,4,8, \ldots, 2^{j}, \ldots$, and $\chi_{M}(\xi)=\chi\left(M^{-1} \xi\right)$, which has the compact support $\frac{M}{2} \leq|\xi| \leq \frac{3 M}{2}$. For $f \in L^{2}(\mathbf{R})$, we define

$$
\begin{aligned}
f_{M} & =\left(\chi_{M}(\xi) \hat{f}(\xi)\right)^{\vee}, \\
f_{\frac{1}{2}} & =(\psi(\xi) \hat{f}(\xi))^{\vee}, \\
f_{<M} & =f_{\frac{1}{2}}+\sum_{K \in 2^{\mathbb{N}}, K<M} f_{K},
\end{aligned}
$$

which defines the decomposition

$$
f=f_{\frac{1}{2}}+\sum_{K \in 2^{\mathbb{N}}} f_{K} .
$$

There holds the almost orthogonality and the approximate projection property

$$
\begin{aligned}
\|f\|_{2}^{2} & \approx \sum_{K \in \mathbb{D}}\left\|f_{K}\right\|_{2}^{2}, \\
\left\|f_{M}\right\|_{2}^{2} & \approx\left\|\left(f_{M}\right)_{M}\right\|_{2}^{2} .
\end{aligned}
$$

The following is also clear for $M \geq 1$ :

$$
\left\|\left|\partial_{v}\right| f_{M}\right\|_{2}^{2} \approx M\left\|f_{M}\right\|_{2}^{2} .
$$

We make use of the notation

$$
f_{\sim M}=\sum_{K \in \mathbb{D}: \frac{1}{C}} f_{K \leq K \leq C M},
$$

for some constant $C$ that is independent of $M$. Generally, the exact value of $C$ being used is not important; what is important is that it is finite and independent of $M$. With this notation we also have

$$
\|f\|_{2}^{2} \approx_{C} \sum_{K \in \mathbb{D}}\left\|f_{\sim K}\right\|_{2}^{2} .
$$


During much of the proof we are also working with Littlewood-Paley decompositions defined in the $(z, v)$ variables, with the notation conventions being analogous. Our convention is to use $N$ to denote Littlewood-Paley projections in $(z, v)$ and $M$ to denote projections only in the $v$ direction.

Another key Fourier analysis tool employed in this work is the paraproduct decomposition, introduced by Bony ([11]) (see also [1]). Given suitable functions $f, g$, we may define the paraproduct decomposition (in either $(z, v)$ or just $v$ ),

$$
\begin{aligned}
f g & =T_{f} g+T_{g} f+\mathcal{R}(f, g) \\
& =\sum_{N \geq 8} f_{<N / 8} g_{N}+\sum_{N \geq 8} f_{N} g_{<N / 8}+\sum_{N \in \mathbb{D}} \sum_{N / 8 \leq N^{\prime} \leq 8 N} g_{N^{\prime}} f_{N},
\end{aligned}
$$

where all the sums are understood to run over $\mathbb{D}$. In our work we do not employ the notation in the first line since at most steps in the proof we are forced to explicitly write the sums and treat them term by term anyway. This is due to the fact that we are working in nonstandard regularity spaces and, more crucially, are usually applying multipliers that do not satisfy any version of $A T_{f} g \approx T_{f} A g$. Hence, we have to prove almost everything "from scratch" and can only rely on standard para-differential calculus as a guide.

We also show some product estimates (or Young's inequality) based on Sobolev embedding. It holds for $s>1$ that

$$
\begin{aligned}
\|f g\|_{H^{s}(\mathbb{T} \times R)} & \lesssim\|f\|_{H^{s}(\mathbb{T} \times R)}\|g\|_{H^{s}(\mathbb{T} \times R)} \\
\|\hat{f} * \hat{g}\|_{2} & \lesssim\|f\|_{2}\|g\|_{H^{s}(\mathbb{T} \times R)} \\
\|\hat{f} * \hat{g} * \hat{h}\|_{2} & \lesssim\|f\|_{2}\|g\|_{2}\|h\|_{H^{s}(\mathbb{T} \times R)}
\end{aligned}
$$

We end this subsection by introducing the commutator estimate which can be found in [27].

Lemma A.1 ([27]). Let $J=(1-\Delta)^{\frac{1}{2}}$; for $1<p<\infty$ and $s \geq 0$ it holds that

$$
\left\|J^{s}(f g)-f\left(J^{s} g\right)\right\|_{p} \lesssim_{p, s}\|\nabla f\|_{\infty}\left\|J^{s-1} g\right\|_{p}+\left\|J^{s} f\right\|_{p}\|g\|_{\infty} .
$$

\section{A.2. Composition lemma}

According to the coordinate transform, we need the following composition lemma.

Lemma A.2. Suppose that $\gamma>1$; let $F \in H^{\gamma}: \mathbb{T} \times \mathbf{R} \rightarrow \mathbf{R}, G: \mathbb{T} \times \mathbf{R} \rightarrow \mathbb{T} \times \mathbf{R}$ be such that $\left\|\nabla G-I_{2 \times 2}\right\|_{L^{\infty}} \leq \frac{1}{4}$ and $\nabla G-I_{2 \times 2} \in H^{\gamma}: \mathbb{T} \times \mathbf{R} \rightarrow \mathcal{M}_{2 \times 2}$. Then there exists $C=C\left(\left\|\nabla G-I_{2 \times 2}\right\|_{H^{\gamma}}, \gamma\right)$ such that

$$
\|F \circ G\|_{H^{\gamma}} \leq C\|F\|_{H^{\gamma}} .
$$

Proof. First, we have $\|F \circ G\|_{2}^{2} \approx\|F\|_{2}^{2}$. Then by the fact that $\nabla(F \circ G)=[(\nabla F) \circ G]$ $\left(\nabla G-I_{2 \times 2}\right)+(\nabla F) \circ G$, we have

$$
\begin{aligned}
\|F \circ G\|_{H^{\gamma}} & \lesssim\|(\nabla F) \circ G\|_{H^{\gamma-1}}\left\|\left(\nabla G-I_{2 \times 2}\right)\right\|_{H^{\gamma}}+\|(\nabla F) \circ G\|_{H^{\gamma-1}} \\
& \lesssim\|(\nabla F) \circ G\|_{H^{\gamma-1}} .
\end{aligned}
$$


Let $\gamma=[\gamma]+\{\gamma\}$ with $\{\gamma\} \in[0,1)$; then by the equivalent definition of the fractionalorder Sobolev spaces we get

$$
\begin{aligned}
\|F\|_{H^{\gamma}} & \approx\|F\|_{H^{[\gamma]}} \\
& +\sum_{\gamma_{1}+\gamma_{2}=[\gamma]}\left(\int_{(\mathbb{T} \times \mathbf{R})^{2}} \frac{\left|\partial_{x}^{\gamma_{1}} \partial_{y}^{\gamma_{2}} F\left(x_{1}, y_{1}\right)-\partial_{x}^{\gamma_{1}} \partial_{y}^{\gamma_{2}} F\left(x_{2}, y_{2}\right)\right|^{2}}{\left(\left(x_{1}-x_{2}\right)^{2}+\left(y_{1}-y_{2}\right)^{2}\right)^{1+\{\gamma\}}} d x_{1} d y_{1} d x_{2} d y_{2}\right)^{\frac{1}{2}} .
\end{aligned}
$$

Therefore, we only need to prove

$$
\|F \circ G\|_{H^{\{\gamma\}}} \leq C\|F\|_{H^{\{\gamma\}}} .
$$

Indeed, we have

$$
\begin{aligned}
&\|F \circ G\|_{H^{\{\gamma\}}}^{2} \approx \int_{\mathbb{T} \times \mathbf{R}} \int_{\mathbb{T} \times \mathbf{R}} \frac{\left|F\left(G\left(x_{1}, y_{1}\right)\right)-F\left(G\left(x_{2}, y_{2}\right)\right)\right|^{2}}{\left(\left(x_{1}-x_{2}\right)^{2}+\left(y_{1}-y_{2}\right)^{2}\right)^{1+\{\gamma\}} d x_{1} d y_{1} d x_{2} d y_{2}} \\
& \lesssim \int_{\mathbb{T} \times \mathbf{R}} \int_{\mathbb{T} \times \mathbf{R}} \frac{\left|F\left(G\left(x_{1}, y_{1}\right)\right)-F\left(G\left(x_{2}, y_{2}\right)\right)\right|^{2}}{\left|G\left(x_{1}, y_{1}\right)-G\left(x_{1}, y_{1}\right)\right|^{2+2\{\gamma\}}} \\
& \times \frac{\left|G\left(x_{1}, y_{1}\right)-G\left(x_{1}, y_{1}\right)\right|^{2+2\{\gamma\}}}{\left(\left(x_{1}-x_{2}\right)^{2}+\left(y_{1}-y_{2}\right)^{2}\right)^{1+\{\gamma\}}} d x_{1} d y_{1} d x_{2} d y_{2} \\
& \lesssim\|\nabla G\|_{L^{\infty}} \int_{\mathbb{T} \times \mathbf{R}} \int_{\mathbb{T} \times \mathbf{R}} \frac{\left|F\left(G\left(x_{1}, y_{1}\right)\right)-F\left(G\left(x_{2}, y_{2}\right)\right)\right|^{2}}{\left|G\left(x_{1}, y_{1}\right)-G\left(x_{1}, y_{1}\right)\right|^{2+2\{\gamma\}}} d x_{1} d y_{1} d x_{2} d y_{2} .
\end{aligned}
$$

By the assumption $\left\|\nabla G-I_{2 \times 2}\right\|_{L^{\infty}} \leq \frac{1}{4}$, we have that $(x, y) \rightarrow(z, v)=G(x, y)$ is invertible and thus

$$
\begin{aligned}
\|F \circ G\|_{H^{\{\gamma\}}}^{2} & \lesssim\|\nabla G\|_{L^{\infty}} \int_{\mathbb{T} \times \mathbf{R}} \int_{\mathbb{T} \times \mathbf{R}} \frac{\left|F\left(z_{1}, v_{1}\right)-F\left(z_{1}, v_{1}\right)\right|^{2}}{\left(\left(z_{1}-z_{2}\right)^{2}+\left(v_{1}-v_{2}\right)^{2}\right)^{1+\{\gamma\}}} d z_{1} d v_{1} d z_{2} d v_{2} \\
& \lesssim\left(\left\|\nabla G-I_{2 \times 2}\right\|_{H^{2}}+1\right)\|F\|_{H^{\{\gamma\}}}^{2} .
\end{aligned}
$$

Thus we have proved the lemma.

Acknowledgments. The work of N.M. is supported by NSF grant DMS-1716466 and by Tamkeen under the NYU Abu Dhabi Research Institute grant of the center SITE.

\section{References}

[1] H. Bahouri, J.-Y. Chemin, and R. Danchin, Fourier analysis and nonlinear partial differential equations. Grundlehren Math. Wiss. 343, Springer, Heidelberg, 2011 Zbl 1227.35004 MR 2768550

[2] J. Bedrossian, M. Coti Zelati, and V. Vicol, Vortex axisymmetrization, inviscid damping, and vorticity depletion in the linearized 2D Euler equations. Ann. PDE 5 (2019), no. 1, Paper No. 4, 192 Zbl 1428.35321 MR 3987441

[3] J. Bedrossian, P. Germain, and N. Masmoudi, On the stability threshold for the 3D Couette flow in Sobolev regularity. Ann. of Math. (2) 185 (2017), no. 2, 541-608 Zbl 1366.35113 MR 3612004 
[4] J. Bedrossian, P. Germain, and N. Masmoudi, Dynamics near the subcritical transition of the 3D Couette flow I: Below threshold case. Mem. Amer. Math. Soc. 266 (2020), no. 1294, v+158 Zbl 1444.35002 MR 4126259

[5] J. Bedrossian, P. Germain, and N. Masmoudi, Dynamics near the subcritical transition of the 3D Couette flow II: Above threshold case. 2015, arXiv:1506.03721. To appear in Mem. Amer. Math. Soc.

[6] J. Bedrossian and S. He, Inviscid damping and enhanced dissipation of the boundary layer for 2D Navier-Stokes linearized around Couette flow in a channel. Comm. Math. Phys. 379 (2020), no. 1, 177-226 Zbl 1448.76057 MR 4152270

[7] J. Bedrossian and N. Masmoudi, Inviscid damping and the asymptotic stability of planar shear flows in the 2D Euler equations. Publ. Math. Inst. Hautes Études Sci. 122 (2015), 195-300 Zbl 1375.35340 MR 3415068

[8] J. Bedrossian, N. Masmoudi, and C. Mouhot, Landau damping: paraproducts and Gevrey regularity. Ann. PDE 2 (2016), no. 1, Art. 4, 71 Zbl 1402.35058 MR 3489904

[9] J. Bedrossian, N. Masmoudi, and V. Vicol, Enhanced dissipation and inviscid damping in the inviscid limit of the Navier-Stokes equations near the two dimensional Couette flow. Arch. Ration. Mech. Anal. 219 (2016), no. 3, 1087-1159 Zbl 1339.35208 MR 3448924

[10] J. Bedrossian, V. Vicol, and F. Wang, The Sobolev stability threshold for 2D shear flows near Couette. J. Nonlinear Sci. 28 (2018), no. 6, 2051-2075 Zbl 1403.35228 MR 3867637

[11] J.-M. Bony, Calcul symbolique et propagation des singularités pour les équations aux dérivées partielles non linéaires. Ann. Sci. École Norm. Sup. (4) 14 (1981), no. 2, 209-246 Zbl 0495.35024 MR 631751

[12] F. Bouchet and H. Morita, Large time behavior and asymptotic stability of the 2D Euler and linearized Euler equations. Phys. D 239 (2010), no. 12, 948-966 Zbl 1189.35234 MR 2639613

[13] P. Braz e Silva, Nonlinear stability for 2 dimensional plane Couette flow. Rev. Integr. Temas Mat. 22 (2004), no. 1-2, 67-81 MR 2344246

[14] Q. Chen, T. Li, D. Wei, and Z. Zhang, Transition threshold for the 2-D Couette flow in a finite channel. Arch. Ration. Mech. Anal. 238 (2020), no. 1, 125-183 Zbl 1446.35094 MR 4121130

[15] Q. Chen, D. Wei, and Z. Zhang, Transition threshold for the 3D Couette flow in a finite channel. 2020, arXiv:2006.00721

[16] M. Coti Zelati, T. M. Elgindi, and K. Widmayer, Enhanced dissipation in the Navier-Stokes equations near the Poiseuille flow. Comm. Math. Phys. 378 (2020), no. 2, 987-1010 Zbl 1446.35095 MR 4134940

[17] Y. Deng and N. Masmoudi, Long time instability of the Couette flow in low Gevrey spaces. 2018, arXiv:1803.01246. To appear in Comm. Math. Phys.

[18] S. Ding and Z. Lin, Stability for two-dimensional plane Couette flow to the incompressible Navier-Stokes equations with Navier boundary conditions. Commun. Math. Sci. 18 (2020), no. $5,1233-1258$

[19] P. G. Drazin and W. H. Reid, Hydrodynamic stability. Cambridge Monogr. Mech. Appl. Math., Cambridge University Press, Cambridge-New York, 1981 Zbl 0449.76027 MR 604359

[20] E. Grenier, T. T. Nguyen, F. Rousset, and A. Soffer, Linear inviscid damping and enhanced viscous dissipation of shear flows by using the conjugate operator method. J. Funct. Anal. 278 (2020), no. 3, 108339, 27 Zbl 1447.35247 MR 4030287

[21] S. Ibrahim, Y. Maekawa, and N. Masmoudi, On pseudospectral bound for non-selfadjoint operators and its application to stability of Kolmogorov flows. Ann. PDE 5 (2019), no. 2, Paper No. 14, 84 Zbl 1439.35370 MR 4015164 
[22] A. Ionescu and H. Jia, Axi-symmetrization near point vortex solutions for the 2D Euler equation. 2019, arXiv:1904.09170

[23] A. D. Ionescu and H. Jia, Inviscid damping near the Couette flow in a channel. Comm. Math. Phys. 374 (2020), no. 3, 2015-2096 Zbl 1468.76025 MR 4076093

[24] A. Ionescu and H. Jia, Nonlinear inviscid damping near monotonic shear flows. 2020, arXiv:2001.03087

[25] H. Jia, Linear inviscid damping in Gevrey spaces. Arch. Ration. Mech. Anal. 235 (2020), no. 2, 1327-1355 Zbl 1434.35079 MR 4064200

[26] H. Jia, Linear inviscid damping near monotone shear flows. SIAM J. Math. Anal. 52 (2020), no. 1, 623-652 Zbl 1431.35089 MR 4062802

[27] T. Kato and G. Ponce, Commutator estimates and the Euler and Navier-Stokes equations. Comm. Pure Appl. Math. 41 (1988), no. 7, 891-907 Zbl 0671.35066 MR 951744

[28] L. Kelvin, Stability of fluid motion-rectilinear motion of viscous fluid between two parallel plates. Phil. Mag. 24 (1887), 188-196

[29] L. Landau, On the vibrations of the electronic plasma. Acad. Sci. USSR. J. Phys. 10 (1946), 25-34 Zbl 0063.03439 MR 0023765

[30] T. Li, D. Wei, and Z. Zhang, Pseudospectral bound and transition threshold for the 3D Kolmogorov flow. Comm. Pure Appl. Math. 73 (2020), no. 3, 465-557 Zbl 1442.35346 MR 4057900

[31] Z. Lin and M. Xu, Metastability of Kolmogorov flows and inviscid damping of shear flows. Arch. Ration. Mech. Anal. 231 (2019), no. 3, 1811-1852 Zbl 1426.76155 MR 3902476

[32] Z. Lin and C. Zeng, Inviscid dynamical structures near Couette flow. Arch. Ration. Mech. Anal. 200 (2011), no. 3, 1075-1097 Zbl 1229.35197 MR 2796139

[33] N. Masmoudi and W. Zhao, Nonlinear inviscid damping for a class of monotone shear flows in finite channel. 2020, arXiv:2001.08564

[34] N. Masmoudi and W. Zhao, Enhanced dissipation for the 2D Couette flow in critical space. Commun. Partial Differ. Equations 45 (2020), no. 12, 1682-1701 Zbl 1462.35247 MR 4176913

[35] C. Mouhot and C. Villani, On Landau damping. Acta Math. 207 (2011), no. 1, 29-201 Zbl 1239.82017 MR 2863910

[36] W. Orr, Stability and instability of steady motions of a perfect liquid. Proc. Ir. Acad. Sect. A: Math Astron. Phys. Sci. 27(1907), 9-66

[37] L. Rayleigh, On the stability, or instability, of certain fluid motions. Proc. Lond. Math. Soc. 11 (1879/80), 57-70 Zbl 12.0711.02 MR 1575266

[38] S. Ren and W. Zhao, Linear damping of Alfvén waves by phase mixing. SIAM J. Math. Anal. 49 (2017), no. 3, 2101-2137 Zbl 1365.76061 MR 3664214

[39] D. Ryutov, Landau damping: half a century with the great discovery. Plasma Phys. Control Fusion 41 (1999), A1-A12

[40] D. Wei and Z. Zhang, Transition threshold for the 3D Couette flow in Sobolev space. Comm. Pure Appl. Math. 74 (2021), no. 11, 2398-2479

[41] D. Wei, Z. Zhang, and W. Zhao, Linear inviscid damping for a class of monotone shear flow in Sobolev spaces. Comm. Pure Appl. Math. 71 (2018), no. 4, 617-687 Zbl 1390.35251 MR 3772399

[42] D. Wei, Z. Zhang, and W. Zhao, Linear inviscid damping and vorticity depletion for shear flows. Ann. PDE 5 (2019), no. 1, Paper No. 3 Zbl 1428.35336 MR 3919496

[43] D. Wei, Z. Zhang, and W. Zhao, Linear inviscid damping and enhanced dissipation for the Kolmogorov flow. Adv. Math. 362 (2020), article ID 106963 Zbl 1437.76010 MR 4050586 
[44] D. Wei, Z. Zhang, and H. Zhu, Linear inviscid damping for the $\beta$-plane equation. Comm. Math. Phys. 375 (2020), no. 1, 127-174 Zbl 1448.76179 MR 4082187

[45] A. M. Yaglom, Hydrodynamic instability and transition to turbulence. Fluid Mech. Appl. 100, Springer, Dordrecht, 2012 Zbl 1312.76020 MR 3185102

[46] C. Zillinger, Linear inviscid damping for monotone shear flows. Trans. Amer. Math. Soc. 369 (2017), no. 12, 8799-8855 Zbl 1372.76045 MR 3710645

Received 3 September 2019; revised 15 February 2021; accepted 3 May 2021.

\section{Nader Masmoudi}

NYUAD Research Institute, New York University Abu Dhabi, P.O. Box 129188, Abu Dhabi, United Arab Emirates; and Courant Institute of Mathematical Sciences, New York University, 251 Mercer Street, New York, NY 10012, USA; masmoudi@cims.nyu.edu

\section{Weiren Zhao}

Department of Mathematics, New York University Abu Dhabi, Saadiyat Island, P.O. Box 129188, Abu Dhabi, United Arab Emirates; zjzjzwr@126.com; wz19@nyu.edu 